\title{
Impact assessment of facial recognition algorithms' performance when modifying nose dimensions
}

Jacob Wolen

Follow this and additional works at: https://researchrepository.wvu.edu/etd

\section{Recommended Citation}

Wolen, Jacob, "Impact assessment of facial recognition algorithms' performance when modifying nose dimensions" (2015). Graduate Theses, Dissertations, and Problem Reports. 6967.

https://researchrepository.wvu.edu/etd/6967

This Thesis is protected by copyright and/or related rights. It has been brought to you by the The Research Repository @ WVU with permission from the rights-holder(s). You are free to use this Thesis in any way that is permitted by the copyright and related rights legislation that applies to your use. For other uses you must obtain permission from the rights-holder(s) directly, unless additional rights are indicated by a Creative Commons license in the record and/ or on the work itself. This Thesis has been accepted for inclusion in WVU Graduate Theses, Dissertations, and Problem Reports collection by an authorized administrator of The Research Repository @ WVU. For more information, please contact researchrepository@mail.wvu.edu. 


\title{
IMPACT ASSESSMENT OF FACIAL RECOGNITION ALGORITHMS' PERFORMANCE WHEN MODIFYING NOSE DIMENSIONS
}

\author{
Jacob Wolen \\ Thesis submitted to the \\ Benjamin M. Statler College of Engineering and Mineral Resources \\ at West Virginia University \\ in partial fulfillment of the requirements \\ for the degree of \\ Master of Science \\ in \\ Computer Science
}

Roy S. Nutter, Ph.D., Committee Chairman

Donald A. Adjeroh, Ph.D.

Thirimachos Bourlai, Ph.D.

Lane Department of Computer Science and Electrical Engineering

Morgantown, West Virginia

2015

Keywords: Facial Recognition, Feature Modification, Nose Modification, Plastic Surgery

Copyright 2015 Jacob Wolen 


\section{Abstract \\ IMPACT ASSESSMENT FACIAL RECOGNITION ALGORITHMS' PERFORMANCE WHEN MODIFYING NOSE DIMENSIONS}

by Jacob Wolen

This work quantitatively measures the impact of modifying the nasal width and length dimensions, in a simulated plastic surgery, on the Facial Recognition algorithms, Principal Component Analysis (PCA), Linear Discrimination Analysis (LDA), and Local Binary Patterns Histogram (LBPH). This was integrated through the use of OpenCV. It was found that as the nose width increases beyond $40 \%$ its original width, there is an average decrease in facial recognition performance of up to $14 \%$. It was also found that as the nose was modified vertically, there was less than a $3 \%$ decrease in performance for the facial recognition algorithms. These rates are consistent with previous research in the field although, these are more quantitative. The experimental structure used is modular in nature and allows for easy insertion of other Facial Recognition Algorithms and other Facial Recognition Datasets. 


\section{ACKNOWLEDGMENTS}

I would like to take a quick moment and thank all of those that have helped and supported me throughout the process of creating my thesis and through my academic career. To my fiancée, Maggie, who has always been there with me, standing by me; helping, encouraging, and pushing me to be the best that I can be (and understanding when I fall short). I would like to give a huge thank you to my mother and sister for helping and supporting me, in all I do, throughout my life. To my advisor and mentor, Dr. Roy S. Nutter, Jr., for all of his guidance throughout this whole process, his invaluable advice, and comments which helped me to shape my ideas. I would also like to thank Dr. Donald Adjeroh and Dr. Thirimachos Bourlai for taking time out of their very busy schedules to help make this thesis the best it could be. A very big thanks to my co-worker, Domenick Poster for all of his help throughout this process; he's advice and suggestions have been invaluable and insightful. I would also like to thank my other co-workers, Elliott Iannello and Jacob Tyo, for all of their support and help throughout preparing this thesis. Finally I would like to thank all of my friends and family for all of their help and support, for which I greatly needed in developing this thesis. 
TABLE OF CONTENTS

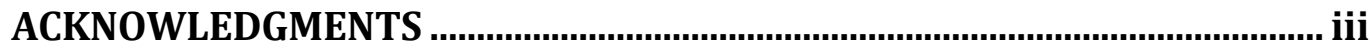

Table of Contents ...............................................................................................

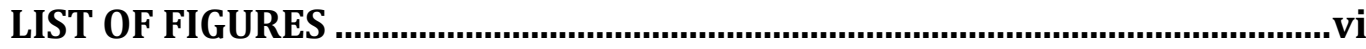

LIST OF TABLES ................................................................................................ viii

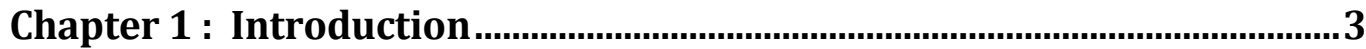

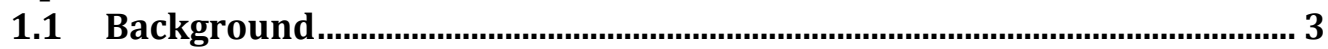

1.2 Motivation ................................................................................................ 4

1.3 Statement of Problem/Research Questions ............................................... 5

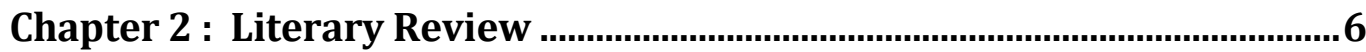

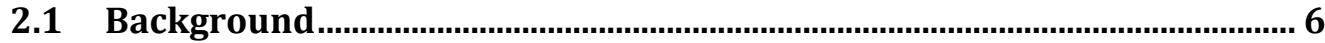

2.1.1 Specification ..................................................................................................................6

2.1.2 Face Recognition Functionality Modes .................................................................6

2.1.3 Face Recognition Basic Procedure ………………………………………………... 6

2.1.4 Face Recognition Sets .......................................................................................... 8

2.1.5 Face Recognition Algorithm (Technique) Categorization...............................9

2.1.6 Face Recognition Common Challenge.....................................................................9

2.2 Facial Recognition Algorithms....................................................................10

2.3 Plastic Surgery and Facial Recognition ....................................................11

2.4 Comparison of Works...................................................................................13

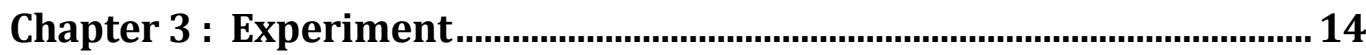

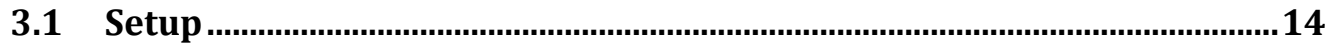

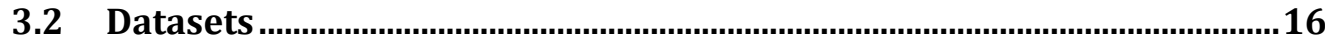

3.2.1 AT\&T (formally the ORL) Dataset...................................................................... 16

3.3 Proposed System...........................................................................................17

3.3.1 Data Generation............................................................................................................ 18

3.3.2 Experiment ...................................................................................................................... 22

3.3.3 Results (Experiment Analysis) ......................................................................... 25

3.4 Evaluation of System ……..........................................................................27

3.4.1 Randomization of the Test (Probe) Set.......................................................... 28

3.4.2 Identity Tests ........................................................................................................... 29

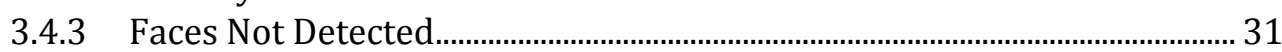

3.4.4 Euclidean Distance...................................................................................................... 32

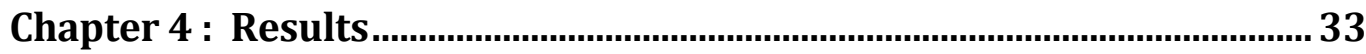

4.1 Faces Not Detected During Trials ...........................................................33

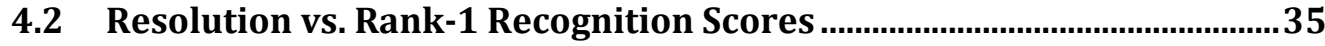

4.3 Image Similarity vs. Rank-1 Recognition Scores.........................................38

4.4 Cumulative Match Curve (CMC) ..................................................................41

4.4.1 CMC Graph Relationships ...................................................................................... 41

4.4.2 Comparing CMC Categories ........................................................................................... 47

4.5 Euclidean Distances and Confidence ............................................................50

4.6 Comparisons with Other Experiments .......................................................53

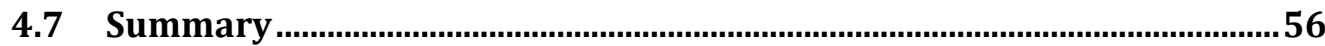




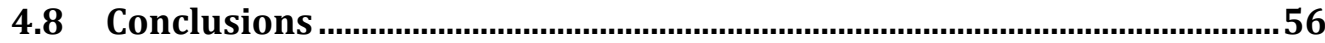

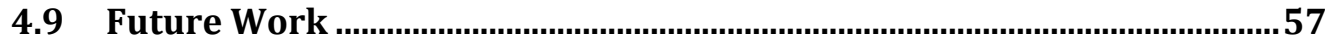

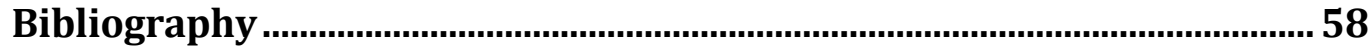

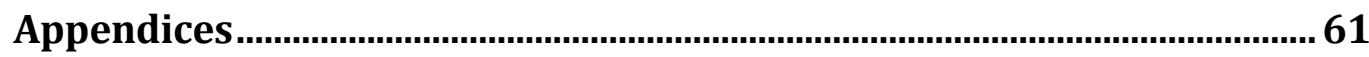

APPENDIX A : Resolution Vs. Rank-1 Recognition Rates.....................................61

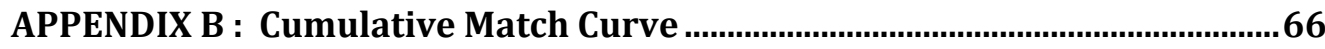

APPENDIX C : Image Similarity Vs. Rank-1 Recognition Rates..........................91

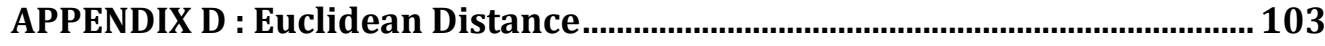




\section{LIST OF FIGURES}

Figure 1: Face Recognition System Basic Procedure for Processing Images [22] .............. 7 Figure 2: General Structure of the AT\&T (formally the ORL) Dataset. There are 40 subjects in the dataset denoted as $\mathrm{s} 1$ to $\mathrm{s} 40$ with 10 images per subject

Figure 3: Visualization of the Experiment Process. Each entity is a distinct tool................ 18

Figure 4: Tree Structure of how the modified images were stored .................................. 19

Figure 5: Process Diagram for generating a dataset full of simulated nose modifications .. 20

Figure 6: Example of how the facial features are detected and modified.......................... 21

Figure 7: Process Diagram for running the experiment trial(s) ......................................23

Figure 8: Parallel List Structure used while running experiment trials. ..............................2 24

Figure 9: Process Diagram of how the Experiment Results Tool will import the experiment results file 26

Figure 10: Class Diagram of the Experiment Results File.................................................26

Figure 11: Visualization of the Results Section Organization ............................................ 33

Figure 12: Faces Not Detected when loading the Intra-Trial Randomized images for the Experiment. Top graph is width modifications, and the bottom is length modifications. 34

Figure 13: Faces Not Detected when loading in the Inter-Trial Randomized images for the Experiment. Top graph is width modifications, and the bottom is length modifications 34

Figure 14: Intra-Trial Randomization, Resolution vs. Rank-1 Recognition Rates (top: Width \&

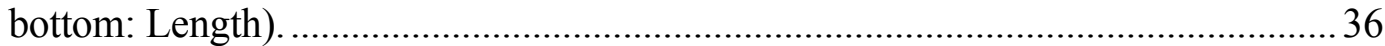

Figure 15: Inter-Trial Randomization, Resolution vs. Rank-1 Recognition Rates (top: Width \& bottom: Length)

Figure 16: LDA Case (A) PSNR vs. Rank-1 Recognition Rates for Intra-Trial Randomization for Width Modifications LDA 38

Figure 17: LDA Case (B) PSNR vs. Rank-1 Recognition Rates for Intra-Trial Randomization for Width Modifications 39

Figure 18: LDA Case (B) PSNR vs. Rank-1 Recognition Rates for Intra-Trial Randomization for Length Modifications 40

Figure 19: Example of a CMC graph where the Baseline (unmodified test images) CMC curve is greater than the Treatment (modified test images) CMC curve (also defined as Category 1). 42

Figure 20: Distribution of which Width Modification falls into the CMC categories described above. 43

Figure 21: Distribution of which Length Modifications falls into the CMC categories described above.

Figure 22: Examples of CMC Graphs for Inter-Trial, Randomly Selecting the test images. (a) Example of Baseline Curve greater than the Treatment (Category 1) (b) Treatment Curve is greater than the Baseline (Category 2). 45

Figure 23: Distribution of which Length Modification falls into the CMC categories for Inter-Trial Randomization. 45

Figure 24: Distribution of which Width Modifications falls into the CMC categories for InterTrial Randomization 46

Figure 25: Intra-Trial Randomization Accumulated baseline and treatment distances for LDA 50 
Figure 26: Intra-Trial Randomization Difference between Baseline and Treatment Accumulated

Distances for LDA. 50

Figure 27: Intra-Trial Randomization Accumulated Baseline and Treatment Distances for LDA 51

Figure 28: Intra-Trial Randomization Accumulated Difference between Baseline and Treatment

Distances for LDA. 51

Figure 29: Inter-Trial Randomization for Accumulated Baseline and Treatment Distances for

LDA. 52

Figure 30: Inter-Trial Randomization Accumulated Difference between Baseline and Treatment

Distances for LDA. 52

Figure 31: Inter-Trial Randomization Accumulated Baseline and Treatment Distances ....53

Figure 32: Inter-Trial Randomization Accumulated Differences between Baseline and Treatment

Distances. 53

Figure 33: Examples of nose modification that extends beyond the width of the face. (a) original

(b) $75 \%$ width modification (c) $100 \%$ width modification 54

Figure 34: Intra-Trial Randomization Accumulated Distance between Baseline and Treatment

Distances for PCA 103

Figure 35: Intra-Trial Randomization Accumulated Difference between Baseline and Treatment

Distances for PCA. 103

Figure 36: Intra-Trial Randomization Accumulated Baseline and Treatment Distances for LBPH. 103

Figure 37: Intra-Trial Accumulated Differences between Baseline and Treatment Distances for $\mathrm{LBPH}$ 104 


\section{LIST OF TABLES}

Table 1: System Information. 14

Table 2: List of Software Used in the Experiment as well as the Package Names (in Fedora) and the Software Version.

Table 3: Categorization of CMC Graphs by the CMC curve orientation for Intra-Trial, Randomly Selected Test Sets.

Table 4: Categorization of CMC Graphs by the CMC curve orientation for Inter-Trial, Randomly Selected Test Sets.

Table 5: Comparison of CMC Rank Values between Intra- and Inter-Trial Randomly Selecting the Test Set where the Baseline CMC curve was greater than the Treatment CMC curve.

Table 6: Comparison of CMC Rank Values between Intra- and Inter-Trial Randomly Selecting the Test Set where the Baseline CMC curve was less than the Treatment CMC curve. 48

Table 7: Average Difference between the Baseline and Treatment for $40 \%$ to $100 \%$ Nose Width Modifications 49

Table 8: Resolution vs. Rank-1 Recognition Rates for Intra-Trial Randomization on Nose Width Modifications for LDA

Table 9: Resolution vs. Rank-1 Recognition Rates for Intra-Trial Randomization on Nose Length Modifications for LDA

Table 10: Resolution vs. Rank-1 Recognition Rates for Inter-Trial Randomization for Nose Width Modifications for LDA.

Table 11: Resolution vs. Rank-1 Recognition Rates for Inter-Trial Randomization on Nose Length Modifications for LDA

Table 12: Cumulative Match Curve For Intra-Trial Randomization on Nose Width Modifications for LDA

Table 13: Cumulative Match Curve For Intra-Trial Randomization on Nose Length Modifications for LDA

Table 14: Cumulative Match Curve For Inter-Trial Randomization on Nose Width Modifications for LDA

Table 15: Cumulative Match Curve For Inter-Trial Randomization on Nose Length Modifications for LDA

Table 16: Cumulative Match Curve For Intra-Trial Randomization on Nose Width Modifications for PCA.

Table 17: Cumulative Match Curve For Intra-Trial Randomization on Nose Length Modification for PCA

Table 18: Cumulative Match Curve For Inter-Trial Randomization on Nose Width Modifications for PCA

Table 19:Cumulative Match Curve For Inter-Trial Randomization on Nose Length Modifications for PCA 82

Table 20: Cumulative Match Curve For Intra-Trial Randomization on Nose Width Modifications for LBPH 86

Table 21: Cumulative Match Curve For Intra-Trial Randomization on Nose Length Modifications for LBPH .86 
Table 22: Cumulative Match Curve For Inter-Trial Randomization on Nose Width Modifications for $\mathrm{LBPH}$

Table 23: Cumulative Match Curve For Inter-Trial Randomization on Nose Length Modifications for $\mathrm{LBPH}$ .90

Table 24: LDA Image Similarity (PSNR) vs. Rank-1 Recognition Rates for Intra-Trial Randomization on Nose Width Modifications 92

Table 25: LDA Image Similarity (PSNR) vs. Rank-1 Recognition Rate for Intra-Trial Randomization on Nose Length Modifications.... 93

Table 26: LDA Image Similarity vs. Rank-1 Recognition Rate for Inter-Trial Randomization on Nose Width Modifications 94

Table 27: LDA Image Similarity (PSNR) vs. Rank-1 Recognition Rates for Inter-Trial Randomization on Nose Length Modifications....

Table 28: PCA Image Similarity (PSNR) vs. Rank-1 Recognition Rates for Intra-Trial Randomization on Nose Width Modifications .96

Table 29:PCA Image Similarity (PSNR) vs. Rank-1 Recognition Rates for Intra-Trial Randomization on Nose Length Modifications. 97

Table 30: PCA Image Similarity (PSNR) vs. Rank-1 Recognition Rates for Inter-Trial Randomization on Nose Width Modifications 98

Table 31: PCA Image Similarity (PSNR) vs. Rank-1 Recognition Rates for Inter-Trial Randomization on Nose Length Modifications.... 99

Table 32:LBPH Image Similarity (PSNR) vs. Rank-1 Recognition Rates for Intra-Trial Randomization on Nose Width Modifications

Table 33:LBPH Image Similarity (PSNR) vs. Rank-1 Recognition Rates for Intra-Trial

Randomization on Nose Length Modifications.... 100

Table 34:LBPH Image Similarity (PSNR) vs. Rank-1 Recognition Rates for Inter-Trial Randomization on Nose Width Modifications 102

Table 35:LBPH Image Similarity (PSNR) vs. Rank-1 Recognition Rates for Inter-Trial

Randomization on Nose Length Modifications. 102 


\section{Chapter 1: INTRODUCTION}

\subsection{Background}

Humans always want to know the identity of others by looking at them. An experiment [31] determined the importance of this desire showing that newborns (one to three day old) vaguely recognize known faces. For newborns to learn this skill so quickly, recognizing a person is a critical function for humans. After 1973, Takeo Kanade extended human recognition to computer by creating the first computer facial recognition system for his Ph.D. thesis work [16]. However easy it is for people, even babies, to recognize known faces, the difficult task of recognition remained mostly untouched in computers until Kirby and Sirovich [17], [30] rejuvenated it with a dimensionality reduction technique (also known as Principle Component Analysis or PCA) nearly 15 years later.

Face Recognition is a form of Biometric Recognition, which is the use of unique characteristic(s) to identify people. Facial Recognition systems allow for a ubiquitous way to collect and analyze faces of people who may or may not have consented to the collection of the person's face. In face recognition, still images or images partitioned from a video stream can be used to determine the identity of that person. Other forms of Biometric Recognition, such as Iris, Fingerprint, or even DNA, generally requires that the person be cooperative and willing to provide these for recognition.

Since Kirby and Sirovich [17], [30] first proposed PCA, newer algorithms investigate ways to improve the recognition performance against environment conditions, e.g. illumination and aging of the person. In contrast, investigating single facial feature performance impacts has not seen as much attention. Through understanding the impact facial features and environment condition have on performance, more robust algorithms can be developed.

One method of testing an algorithm against the change of a single facial feature is to use a before and after image(s) in which a facial feature is changed by plastic surgery. Another method would be to use a picture, simulate the surgery on a digital image, show what the person could look like afterwards, and compare those two images. In either case, simulated or real, facial features would be quantized and evaluated. This would allow as well, testing and evaluation of obstructions or masks, added to the face picture as well. Some, like Singh et al. [29], [28], have briefly discussed the impact with respects to real plastic surgery photos. The impact discussed by Singh et al. [29], [28], used multiple plastic surgery procedures and evaluated facial recognition algorithm performance. After Singh, others ([9] and [18]) focused on particular sets of procedures that could be applied to a facial feature, like just to the nose. 
Plastic surgery can be applied to many regions and features of the face, for example, the eyelids, nose, and ears. The nose is one of the features noticeable to humans when interacting in person. Sometimes a person is identified because "they have a (big/small/crooked) nose".

People seek plastic surgery for their nose for either functionality or beauty enhancements to fix the shape of their nose. In 2014 a survey from the American Society of Aesthetic Plastic Surgery [10] indicated it was the second most performed surgery on the face. Singh et al. [28] grouped Plastic Surgery into two broad facial reconstruction categories: local and global. Local plastic surgery is meant to maintain the overall appearance of the facial structure, while focusing on aesthetically changing the feature. Global plastic surgery is usually concerned with functionally changing the feature by restructuring the underlying bone structure. Multiple features/regions usually have slight modifications due to global plastic surgery procedures. Plastic surgery to alter the nose is known as rhinoplasty. Rhinoplasty falls under the category of local plastic surgery procedures.

\subsection{Motivation}

Between safer and quicker recovery time, the number of Plastic Surgery procedures has been increasing from, 1997 and 2014 [10]. With the increase in the numbers of procedures performed each year, it would be instructive to know how much certain features can change before the person is no longer recognizable using standard facial recognition algorithms.

Succinctly put by Singh et al. [29], there are two major problems currently preventing additional study of the Plastic Surgery's impact on Computer-Based Face Recognition.

- "Due to the sensitive nature of the process and the privacy issues involved, it is extremely difficult to prepare a face database that contains images before and after surgery."

- "After surgery, the geometric relationship between the facial features changes significantly and there is no technique to detect and measure such type of alterations."

This thesis will limit its investigation to the impact of the geometry of the nose upon standard facial recognition algorithms. This investigation will use the standard, and well-known algorithms, Principal Component Analysis (PCA), Linear Discriminant Analysis (LDA), and Local Binary Histograms (LBPH).

The nose was selected as the feature upon which to focus, since it is a common and obvious human facial feature. In plastic surgery, Rhinoplasty is a standard and widely performed procedure. This type of surgery has increasing since 1997. Finally since Rhinoplasty is categorized as a local plastic surgery procedure, the overall appearance of the face is preserved. 


\subsection{Statement of Problem/Research Questions}

This thesis will investigate the impact of altering vertical and horizontal nose dimensions on Facial Recognition Algorithms' success rate using 2D photographs. The vertical and horizontal nose dimensions will represent a simulated plastic surgery procedure of varying discrete quantities to the person's original nose. It will answer two questions, the first of which is at what level of nose modification will the recognition rate decrease by $10 \%$ ?

Previous research into plastic surgery and facial recognition reported about a $14 \%$ decrease in recognition. Setting the threshold slightly lower, at $10 \%$, it ensures that the point at which the decrease happens will be captured.

The second question is at which point nose modification negatively impacts the rate of facial detection?

Facial detection is the first step in the facial recognition process, so if it is negatively impacted then fewer images will be factored into the recognition rates. It will be important to know if there is a threshold at which nose modification makes the face not only unrecognizable but also undetectable. 


\section{Chapter 2 : LITERARY REVIEW}

This chapter provides a review of current research in the field of Rhinoplasty and Facial Recognition Systems. Section 2.1 defines the basics of Face Recognition and the components that go into it. Section 2.2 discusses the Facial Recognition Algorithms used. Section 2.3 summarizes the impact plastic surgery has had on the facial recognition algorithm's recognition performance. Section 2.4 draws distinctions between the literature and this work.

\subsection{Background}

\subsubsection{Specification}

A common definition for Face Recognition was generally stated by Zhao et al. [37] as, "given still or video images of a scene, identify or verify one or more persons in the scene using a stored database of faces".

In this generalized statement, Facial Recognition can be further explained as a process in which a face is detected and identified.

\subsubsection{Face Recognition Functionality Modes}

When face recognition is applied to still or video images, it will either try to identify or try to verify the identity of the person in the image. These two operations are called, functionality modes of the system. In both modes, the goal is to detect the person in the image, then compare the extracted face to a stored database of known faces to identify that person.

According to Li and Jain [22], verification mode is a mode in which the claimed identity and incoming image matches the stored identity's face in the face recognition database. The goal for identification is more general than in verification, the goal is to determine if the person is in the stored database and who the person is [22]. In these modes, verification is also known as a one-toone match, whereas identification is a one-to-many matching mode.

\subsubsection{Face Recognition Basic Procedure}

Each face recognition system has a generic set of steps to follow regardless of the Functionality Mode. This procedure was distilled into the image presented in Li and Jain's Handbook to Face Recognition as seen in the image. 


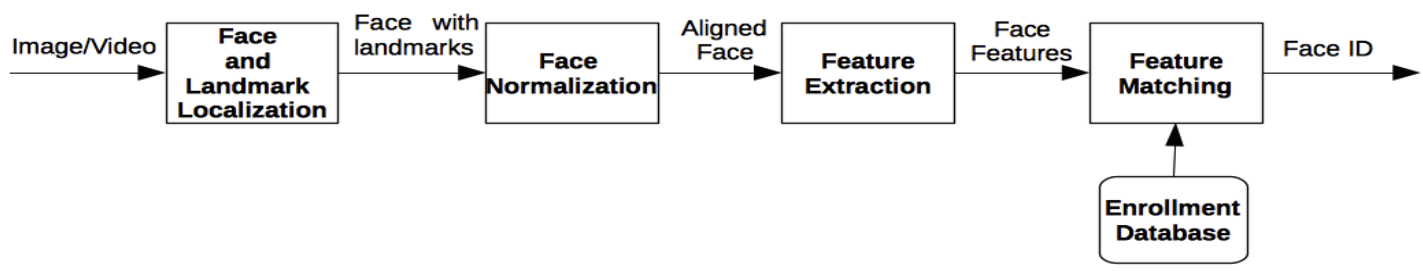

Figure 1: Face Recognition System Basic Procedure for Processing Images [22]

Face and Landmark Localization is the first step applied to still images or video images and is sometimes referred to as the face detection step. In this step, the image's background is separated from the face of the person in the image. If there is no face in the image, then the image can be disregarded. However if a face is detected, then the image and the location of the face are passed to the Face Normalization Step. Face Normalization takes the marked landmarks of the face from the Localization step, and standardizes the face to the enrollment database. To standardize the face, the face is cropped from the image. The pupils of the eyes of the cropped face are detected, and horizontally leveled (aligned). The horizontal leveling ensures that all faces are in the same position, even if the face is twisted to the side. Once the face is geometrically normalized, the face is converted to gray scale to standardize the intensity of the face (including color and illumination value) into a single value. Once finished, the normalized face is passed to the Feature Extraction step.

In the Feature Extraction step, the normalized face is processed for information about the face, like the nose, eyes, and mouth may be extracted. Zhao et al. [37] described feature extraction in more detail as having three different approaches.

1) Appearance-based Approach - A holistic approach in which sub-windows of the whole face are scanned for facial features.

2) Feature-based Approach - A structured classifier that is based on local detected features in the face, along with feature locations and statistics.

3) Hybrid-based Approach - A combination of both Appearance- and Feature-based approaches that Zhao et al. [37] argue is closer to the method that humans use in human face recognition.

The Feature Matching step then uses these extracted features and compares them to a database of known faces (e.g. the Enrollment Database, or Gallery Database). As the extracted features are compared, an identity may be found that match these features. If so then the identity is returned. After this process, if the Face Recognition System is in a Verification mode, then the returned identity can be compared to the claimed identity to see if they match. 


\subsubsection{Face Recognition Sets}

A part of the process described in the Basic Procedure (Figure 1) includes a dataset of images to compare incoming images against. From an extensive number of papers and the Handbook of Face Recognition [22], three types of sets are commonly referred to as generic, gallery, and probe sets. All of these sets are distinct, and in some cases, including Singh et al. [29], [28], Erdogmus et al. [9], and Kose et al. [18], additional datasets are included to add in robustness of the face recognition algorithms.

In a chapter from the Handbook of Face Recognition, Gross et al. [11] look at illumination in images. To get a better accuracy, a generic, gallery, and probe set were used for their algorithm.

- Generic Set: A set of images for an algorithm to train on, which will be used to develop a generic face.

- Gallery Set: Using the generic face from the Generic Set, the gallery (i.e. the Enrollment Database) contains a set of known faces of people that is to be recognized by the algorithm.

- Probe Set: A set of images that have not been trained on or used for the generic set. This set is compared against the Gallery set to see if the algorithm knows the face. The probe set is also referred to as the test set.

In these sets, it appears some literature uses all three, while combining the generic and gallery set under the term, training set. In one particular example, Gross et al. [11] use a training set of independent images to generate a generic face. The generic face is then applied to the face recognition algorithm for increasing the accuracy of distinguishing between other known faces in the Gallery. When the testing set is presented, if the person exists in the gallery then face recognition should be able more accurately determine which person it is.

In other literature, only the training and the testing set are used by the face recognition systems. For example, Belhumeur et al. [2] considered a testing and the training sets. In other literature ([29], [28], and [18]), the experimental construction only mentions two sets. Usage of the three datasets (generic, gallery, and probe/testing) is an optimization for robustness of algorithms that are not always considered, or needed.

Another aspect concerning the number of images in the training and testing set has been looked at in Bourlai et al. [4]. Bourlai et al. [4] considered a computationally restricted device, the Smart Card, and used it to perform Face Verification. The results indicate that the performance of the system levels off, and increasing the number in the training set, could in fact decrease the performance. Although face verification restricts the number of test (probe) images that should be checked, facial recognition training size still needs to be considered when setting up the experiment. 


\subsubsection{Face Recognition Algorithm (Technique) Categorization}

Starting with the seminal work by Kanade in 1973 [16], numerous algorithms and approaches have been suggested improving in accuracy and robustness of environment conditions for acquiring input face images. The algorithms are applied during the Feature Matching Step (Figure 1) on the enrollment database, using the extracted facial features, to determine the identity of the face.

Singh et al.'s paper [29] build upon Zhao et al.'s [37] categorization of face recognition algorithms to define three categories: Appearance-, Feature-, and Texture-based approaches.

Appearance-based algorithms focus on the total combination of features of the face. As Singh et al. [29] stated it, "Appearance-based algorithms ... usually rely on the global semblance of features". This differs from Feature-based algorithms in that, Feature-based algorithms usually determine relationships between facial features, then try to match the pattern. Finally Texturebased algorithms differ from both Appearance- and Feature-based algorithms in that the texture of the face is classified then the texture is matched.

\subsubsection{Face Recognition Common Challenge}

One goal of proposed Face Recognition algorithms is generally to work in multiple environments and varying conditions. Variables that the algorithms must consider are categorized by Zhao et al. [37], as Aging, Pose, Illumination, and Expression (A-PIE). As new facial recognition algorithms are developed, initial consideration for these conditions has become necessary.

The aging of the face has an effect on facial recognition since as a person ages, the face could start having wrinkles, hair color can change, and even scars could appear on an aged face. Although some algorithms have been proposed to handle aging, the impact and unpredictability of how the face ages has been a difficult problem. There is a need to reduce the potential for aging to impact the results of the facial recognition algorithm.

The pose of the face is simply the angle of tilt of the face. By varying the tilt angle of the face, features and textures might not be captured in the image, geometric relationships between features might not be properly measured, or the feature extraction might not detect the features since they are not where they are expected to be at. For this reason, many techniques have arisen to address the pose problem. In addition, face normalization is an essential step to the facial recognition algorithm. Pose correcting algorithms can be complex mathematical functions dependent on various detected facial features, or computationally intensive techniques to manipulate the image. Complex mathematical functions (e.g. the affine transformation) and others were developed to warp the angle of the face in the image while not losing information in the transformation. In the 
physical world, this is similar to turning a photo. A recent technique was developed that morphs a generic face model and generates the dimensions extracted from the photos.

Illumination in the A-PIE problem deals with the amount of light captured in a photo adding to the level of variation for images. In some algorithms, the variation of the pixels (possible due to illumination) is categorized and distinguished between different known faces. Usually Illumination requires a standardized amount of lighting for both the training (gallery) and testing set, however not all images can have controlled lightning. As the compliment to illumination, occlusion, something covers part of or all of the face makes recognition more difficult. In the basic procedure for facial recognition systems, illumination is managed by step "Face Normalization". During this step, the pixels are converted into a standard gray scale, which is meant to be an intensity-based image without color. When dealing with illumination and occlusion in Linear Subspace Algorithms, like PCA and LDA, some variation in illumination and occlusion can be handled if in the training set various levels of illumination and occlusion is included. Some algorithm categories, like Feature-based algorithms, might be more robust to illumination and occlusion as long as critical features on the face are not blocked or distorted.

In the final core problem of Face Recognition algorithms, Expression of the face will change how certain features on the face are oriented, such as the mouth positioning, or openness of the eyes. For this problem, multiple techniques have been proposed to distinguish between and handle identification of an individual. For early algorithms, the necessity of providing a range of various emotions for individuals is needed in the training set in order for the algorithm to accurately distinguish between the people in the gallery set.

\subsection{Facial Recognition Algorithms}

Facial Recognition Algorithms can be broken down into three categories: Appearance-based, Feature-based, and Texture-based algorithms. For this work, two Appearance-based and one Texture-based algorithms will be examined.

Belhumeur et al. [2] were the first to propose LDA, also known as Fisherfaces, by applying Fisher Linear Discriminants to PCA to increase the performance and reduce the complexity of a high dimensionality face. High dimensionality faces are due to the number of pixels in the image that could contain a face as well as the pixel values. Fisherfaces fundamentally work by finding the linear subspaces (e.g. classes) that maximize the distance between clusters of facial components, while minimizing the distance within the clusters. Some component classes could consist of an average illumination level, common expressions, and aging marks (e.g. wrinkles) to name a few.

Although LDA maximizes the distance between clusters, the faces that one wishes to be verified or identified need to be in similar conditions as the training images. For example, if all of the images are well illuminated, then one component that can be factored out is the average intensity 
due to illumination of all of the photos. If an image is presented to LDA which is poorly illuminated, then much of the variation measured by LDA would probably be due to this. The image might not then be identified properly. However if the variations of illumination are in the training images, then LDA will normally handle this variation. LDA will handle the variations of illumination by grouping it as one component to consider for all subjects. Then a standard illumination will be considered for the images and the large variations of the cluster of images might be one discriminant component. In contrast, PCA tries to maximize the scatter of variation between faces. PCA will not make a distinction in the variations and distinguishing components of the face. Thus identifying a specific face might be solely based on the illumination.

Previous research has found that PCA can be more accurate than LDA when the training set and number of classes is small [23]. However, when larger numbers of images are used to train the algorithms, LDA outperforms PCA [3]. One study by Chen used the AT\&T dataset, which is the one being used for this research, and found that when 5 images are used to train, both LDA and PCA had fairly high results [6]. PCA had an 85.25\% recognition rate and LDA had an 87.08\% recognition rate [6].

Local Binary Patterns Histograms (LBPH) is a texture-based algorithm. The texture is made up of elements such as lines, edges, spots and corners in an image. It works by taking the local pixels in each area of an image and converting them into a binary pattern for that area of the image based on how many texture elements are present. Those patterns are then spatially grouped together, as was suggested by Ahonen et al. [1]. After being grouped, the image is represented by a series of binary histograms. This set of histograms becomes the signature for that particular image. The signature is compared against those in the training set to determine a match. Jain states that "The success of LBP in face description is due to the discriminative power and computational simplicity of the operator, and its robustness to monotonic gray scale changes caused by, for example, illumination variations. The use of histograms as features also makes the LBP approach robust to face misalignment and pose variations.’[22].

\subsection{Plastic Surgery and Facial Recognition}

As stated in the Problem Statement, the impact of nose modifications on facial recognition algorithms is being examined. In the medical industry, a modification of the nose is called "rhinoplasty". The American Society for Aesthetic Plastic Surgery found in 2014 [10] that rhinoplasty was the second most common procedure performed on the face. Rhinoplasty can alter the width, length, shape, alignment, and structure of the nose for both cosmetic and functional purposes.

Normally, in order to test the Problem Statement, a subject would need to undergo a rhinoplasty procedure to test the impact of nose modifications on facial recognition algorithms. Originally [29], [28], pre- and post-operation photos would need to be taken in order to test this problem. 
Others have simulated the procedure by implementing various algorithms focusing on making more realistic and controlled alterations to the nose through manipulating the pixels of the picture. Some algorithms, like that of Face-off [7] and other tools [24], guide both plastic surgeons and patients on what the desired post-surgery face would look like in a 2D photo. Then face recognition was used to detect the facial features and allow for manual and automatic feature alterations. Other algorithms, [20] and [21], relied more heavily on 3D information to simulate what the patients and surgeons should expect to see after the surgery.

In 2009, Singh et al. [29] originally showed the need for research in a subfield of Face Recognition, which considered the case of modifying facial features. Singh evaluated both local and global plastic surgery procedures on six facial recognition algorithms. As the name implies, local plastic surgery only impacts one particular facial feature, whereas global plastic surgery will change the underlying structure of the face, and will change multiple facial features. Rhinoplasty typically is classified as local plastic surgery. Out of the six facial recognition algorithms evaluated, Singh et al. [29] reported that LDA had a $22.1 \%$ verification accuracy for Rhinoplasty and a $20.4 \%$ accuracy for all the types of plastic surgery procedures they tested.

After opening up the field of Plastic Surgery to Facial Recognition, Singh et al. [29] added to the field by enumerating the challenges of the field. One challenge Singh et al. addressed was the lack of a Facial Recognition Dataset containing pre- and post-plastic surgery images. Singh et al. collected a dataset of 506 individuals composed of both pre- and post-operation photos. With the construction of this dataset, Singh et al. addressed the second contribution of their paper: a methodology for determining the identification accuracy of the six algorithms tested [29].

In 2012, Erdogmus et al. [9] extended the field by looking at nose alterations with a different dataset and other face recognition algorithms. In "Impact Analysis of Nose Alterations" [9], Erdogmus et al. use a newer publicly accessible dataset (FRGCv1.0) in which a simulated rhinoplasty procedure was applied to all of the individuals in the dataset. The FRGC v1.0 contained both 2D images and simulated 3D models with the same information. For consistency, the $3 \mathrm{D}$ model of the individual was used and a $2 \mathrm{D}$ equivalent transformation was applied to the respective image.

Erdogmus et al. [9] and Kose et al. [18] use simulated rhinoplasty to alter the photos of the people in their dataset and had more control than Singh et al. [29] had for collecting images. Singh et al. ([29] and [28]) gathered real photos of plastic surgery procedures, some of which are rhinoplasty, and tested those. Kose et al. [18] and Erdogmus et al. [9] followed a more randomized approach as proposed by Rabi et al. [26], in which one person's nose is used to replace another person's nose, versus 3D simulated procedures like that of Lee et al. [19]. 
The results from Erdogmus et al. [9], Kose et al. [18], and Singh et al. [29] and [28], all show that if a person changes their nose with plastic surgery then facial recognition has a harder time recognizing them. In other words, the facial recognition performance decreases when a person has plastic surgery. For all of the research from Erdogmus et al. [9], Kose et al. [18], and Singh et al. ([29] and [28]), the amount of plastic surgery is not quantified. So it is not known if small changes to the nose or other plastic surgery procedures produce this decrease in performance or only more drastic plastic surgery does. It would seem more changes produce a bigger decrease in performance, however that is an extrapolation from human recognition to computer facial recognition. No research was found addressing this intuitive relationship.

\subsection{Comparison of Works}

Erdogmus and Singh show the impact of nose modifications on Face Recognition Algorithms. This thesis however is focused on a more quantitative procedure giving controlled changes to the nose and its impact on Facial Recognition performance.

In the previous literature, Singh et al. [29], [28] talks about the impact plastic surgery has, including Rhinoplasty procedures, but does not report the amount of change to the nose. Erdogmus et al. [9] builds off of Singh by simulating a Rhinoplasty procedure for their subjects. They did not report any results regarding the amount of change for each person.

This thesis will quantitatively determine the amount of change a simulated plastic surgery procedure on the nose has on the recognition performance of three standard Facial Recognition Algorithms, Principal Component Analysis, Local Binary Patterns Histograms, and Linear Discriminant Analysis. 


\section{Chapter 3 : EXPERIMENT}

This chapter describes the structure, setup, and running of the tools used in the experiment. The dataset structure is described and any additional information about the dataset used. To conclude the chapter, the framework for running, and collecting the results from the experiment will be discussed.

\subsection{Setup}

For the investigation of this problem, Table 1 describes the environment used to develop and run the experiment.

\begin{tabular}{|l|l|}
\hline Operating System & Fedora $^{\circledR}{ }^{1} 21$ \\
\hline System Type & $32-$ Bit \\
\hline Memory & $3 \mathrm{~GB}$ \\
\hline Processor & Intel ${ }^{\circledR}$ Core ${ }^{\text {TM }}$ i5-2300 CPU @ $2.80 \mathrm{GHz}$ \\
\hline Hard Drive & $15.00 \mathrm{~GB}$ \\
\hline \multicolumn{2}{|c}{ Table 1: System Information }
\end{tabular}

Fedora was chosen to be the host Operating System of this experiment, since it provided a convenient way to install the most recent stable release of the OpenCV (Open Computer Vision) Package, version 2.4.9. [14] Another guiding factor was personal familiarity to the Operating System, so the transition to the development environment was seamless.

Within the environment, a few tools were installed. The entire set of tools that have been composed are written in the Python $\left(^{2}\right.$ programming language version 2.7.8. Python was selected for its ease of use, and speed to develop applications. Similar to the reason for selecting Fedora to be the test environment, prior knowledge and familiarity with the development language already existed.

It will be assumed that Fedora 21 is installed already, for details on installing Fedora, please reference Fedora's Installation Instructions ${ }^{3}$. Once the host Operating System is installed, Python and some of the tools that are used need to be installed.

\footnotetext{
${ }^{1}$ Fedora and the Infinity design logo are trademarks of Red Hat, Inc.

${ }^{2}$ Copyright (C) 2001-2015 Python Software Foundation\%3B All Rights Reserved

${ }^{3}$ https://docs.fedoraproject.org/en-US/Fedora/21/html/Installation_Guide/index.html
} 


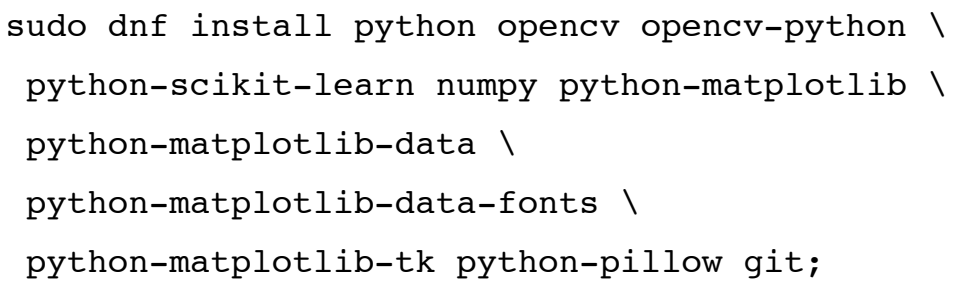

The Software versions that were installed and run are in the following table.

\begin{tabular}{|lll|}
\hline Software Package Name & Fedora Package Name & Version \\
\hline Python & Python & 2.7 .8 \\
\hline OpenCV & Opencv & 2.4 .9 \\
\hline SciKit Learn & opencv-python & 2.4 .9 \\
\hline NumPy & python-scikit-learn & 0.15 .2 \\
\hline matplotlib & Numpy & 1.8 .2 \\
\hline matplotlib & python-matplotlib & 1.3 .1 \\
\hline matplotlib & python-matplotlib-data & 1.3 .1 \\
\hline matplotlib & $\begin{array}{l}\text { python-matplotlib-data- } \\
\text { fonts }\end{array}$ & 1.3 .1 \\
\hline Python Imaging Library (PIL Fork) & python-matplotlib-tk & 1.3 .1 \\
\hline Git & python-pillow & 2.6 .2 \\
\hline & Git & 2.1 .0 \\
\hline
\end{tabular}

Table 2: List of Software Used in the Experiment as well as the Package Names (in Fedora) and the Software Version.

As Table 2 lists, multiple software packages are used. OpenCV with python hooks to the C++ OpenCV implementation was used for face detection and feature extraction in the Experiment, Section 3.3. Since OpenCV only returns Rank-1 results and OpenCV is in $\mathrm{C}++$, a ported version of the OpenCV Algorithms is used for this experiment which ported the language of the Facial Recognition Algorithms from C++ to Python as well as allowing multiple Ranks to be allowed, which was ported by an OpenCV contributor named Philipp Wagner. To get Philipp's port, the following commands will need to be run.

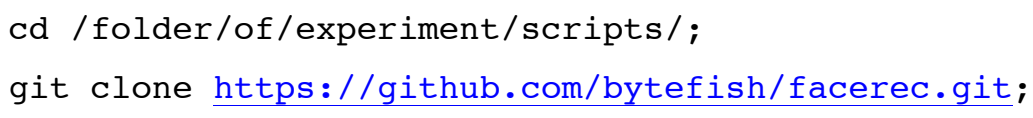


Once these commands have been run, Wagner's port will be available for the experiment. In addition to the additional face recognition package, the Python Imaging Library (Pillow) $\mathbb{C}^{4}$ is the module used to perform the nose modifications with. Scikit-learn [25] is used to split the experiment images into training and testing sets, which is where the Inter- and Intra-Trial Randomization factor in. Matplotlib [12] is used to generate most of the Graphs seen in Chapter 4. Finally NumPy [33] is used for storing the loaded in images of the faces as well as calculation tools in the results of Chapter 4.

Special Consideration should be taken if the standard paths for the tools described in Section 3.1. For example, if the Haarcascade Classifier files are not in their standard path, in /usr/share/OpenCV/haarcascades/, then the file paths will need to be set manually in the source code. If not, the observed issue will result in a lack of any feature detection from OpenCV, since the files could not be loaded successfully.

\subsection{Datasets}

\subsubsection{AT\&T (formally the ORL) Dataset}

The AT\&T dataset was created from 1992 to 1994, as a part of project conducted by the Cambridge University Engineering Department. Ferdinando and Harter [27] describe this dataset as containing images of faces from either students at Cambridge or from Olivetti Research Ltd (ORL), thus the reason for its alternative reference, the ORL Dataset. This data set was selected because it is openly available for use in research. The images are small enough that they did not slow down the experiment. Larger images would slow the experiment in such a way that would not allow for a workable timeline for completing the research. Additional the AT\&T dataset was used to initially assess the impact of facial recognition algorithms with the hope that future research on other datasets will be used (see Future Work).

The dataset contains 400 grayscale images of 40 individuals, 10 photos each. Each set of individual's photos is separated into distinct folders with the naming convention of 'sX', where 's' indicates subject number $\mathrm{X}$ (since there are 40 , s1 through s40 are used). Each image has the naming convention of 'Y.pgm' in each subject's folder, where $Y$ is a number assigned to the image (between 1 and 10). The numbering of each individual's photos has no meaning except for uniqueness to the Operating System's File Structure.

The break down of the structure of the AT\&T Dataset is as follows

\footnotetext{
${ }^{4}$ Copyright 1995-2015, Fredrik Lundh and Contributors, Alex Clark and Contributors. Revision 7729d889 https://pillow.readthedocs.org/en/3.0.x/
} 


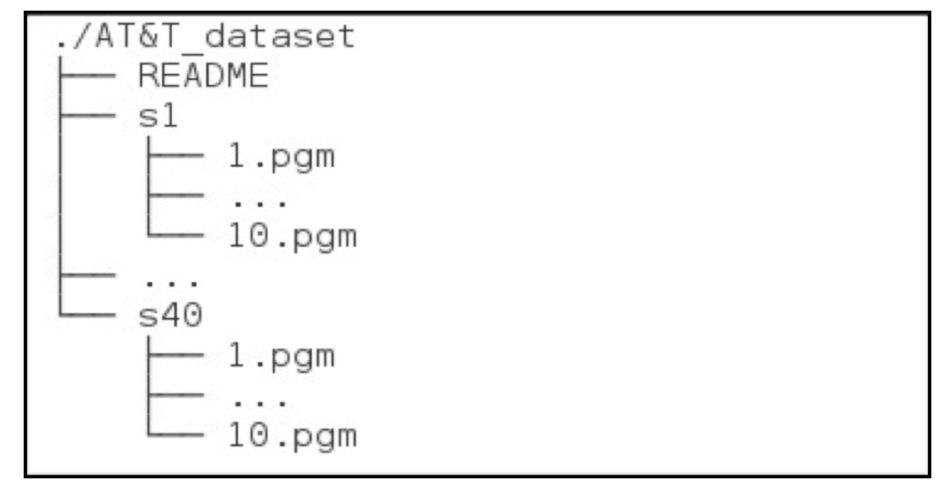

Figure 2: General Structure of the AT\&T (formally the ORL) Dataset. There are 40 subjects in the dataset denoted as s1 to s40 with 10 images per subject

The format of each image is PGM, which stands for Portable Graymap Format and allows for easy exchange of images between various computer systems. For uniform image collection, each image was constrained to $92 \times 112$ pixels, with 8 bits per pixels (or 256 variations of gray). For consistency, the images have only one face per image where each face is in a vertical frontal position. The final constraint is that each photo has the same smooth, dark background color behind each individual.

Some constraints not imposed on the dataset is a lack of control on variation to lighting, expression, time, and facial details. Variations to Facial details refers to some images of subjects might be wearing glasses, while the rest of the images they are not. Time was another constraint since the collection of photos is taken over two years.

Due to the number of photos, quality of the photos (consistent image size), variation between photos, and frequency of use by other researchers, the AT\&T dataset was selected for the evaluation of the simulated nose modifications impact on LDA, PCA, and LBPH.

\subsection{Proposed System}

Testing the impact of nose modifications for LDA, PCA, and LBPH can be broken down into three general tasks: preparing the data, running experiments on the data, and analyzing the results of the data. These three steps can be visualized as Figure 3. 


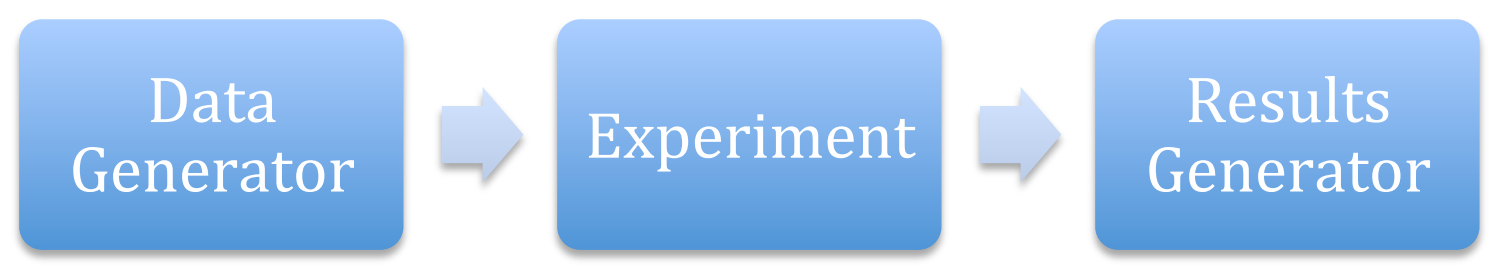

Figure 3: Visualization of the Experiment Process. Each entity is a distinct tool.

In these three steps, each task was separated as a distinct tool in the framework for testing. These tasks were named for their primary functionality: Data Generator, Experiment, and Results. By isolating the three tasks into separate tools, they allow functionality isolation in programming language implementation, procedure, and in their varying dataset structures. For example, the process of creating simulated datasets from the original dataset does not rely on a particular implementation.

One aspect that must be considered initially is how the control on nose modifications will be applied. Since both the width of the nose and the length of the nose will be tested in this work, modifications of increments of 5\% will be tested and examined. The percentage change in the nose is the total length (in pixels) of the original person's detected nose. By using a percentage, variations in resolution for an image are scaled to the resolution. As for the total amount of width or length variation that will be tested, no other works examined this problem, let alone in this fashion. The width of the nose will be tested for up to half the original width of the nose all the way up to twice the size of the original nose. To ensure clarity, if the original nose is at $0 \%$ modified (or no change/original), then a $+100 \%$ increase to the width would be doubling the nose. In contrast to shrink the nose, a modification of $-100 \%$ would need to be applied. Unlike the width, the length of the nose is more restricted, in that at a point the nose would be so long that it would start to impede other facial features like the mouth. So the length of the nose was kept as elongating or shortening the nose by a fifth of its original size (or $\pm 20 \%$ ).

Since each task for examining the nose modification effect have many sub-steps, each step is examined individually over the next few sub-sections. Briefly stated, the Data Generation step focuses on generating all of the various levels of modification for each image in a dataset; the Experiment step takes the various levels of modifications and runs multiple trials on them and generates results; the Results (Experiment Analysis) step takes the generated results and generates graphs of the results produced during the experiment trials.

\subsubsection{Data Generation}

The data generator runs a digitally simulated nose modification on all of the images in the dataset to allow for any dataset to have controlled digital nose modifications added to it. For this 
experiment a dataset, the AT\&T dataset, was selected and does not have an alternative dataset that contains controlled nose modifications. In producing a dataset with all of the nose modifications, an empty structure is first produced that is similar to the original structure. The only difference between the two resides in that each modification dataset is nested in a larger folder holding all variations to the original. For illustrative purposes, Figure 4 shows an example of what the modification dataset(s) would look like.

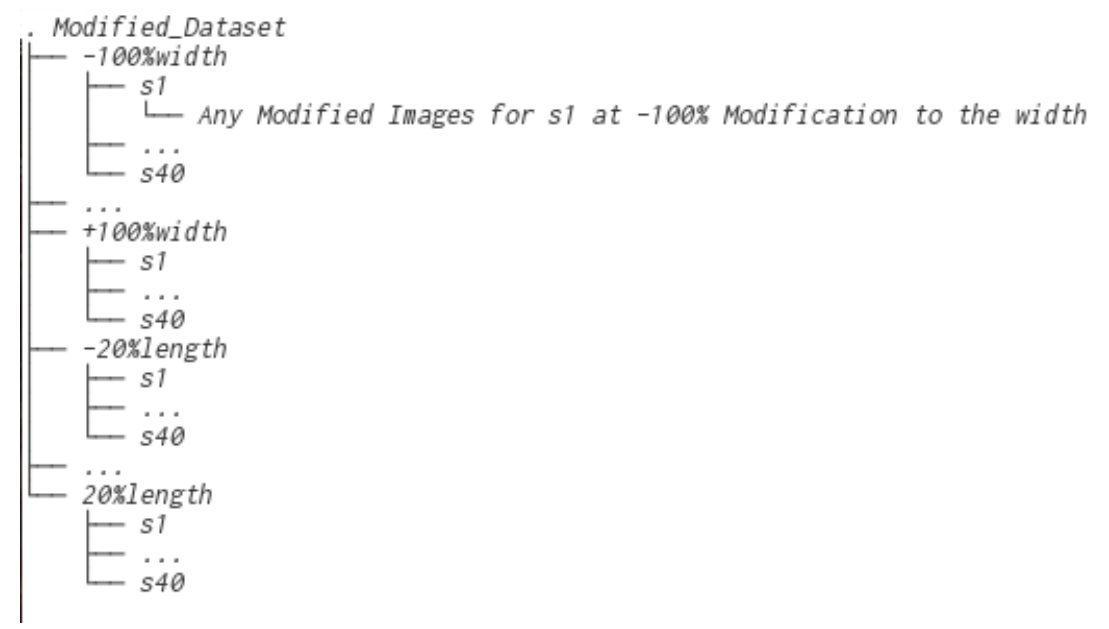

Figure 4: Tree Structure of how the modified images were stored

Once the empty dataset structure is created, the process for reading in the images of the dataset, modifying the nose by a set amount, and storing them can be seen in Figure 5. 


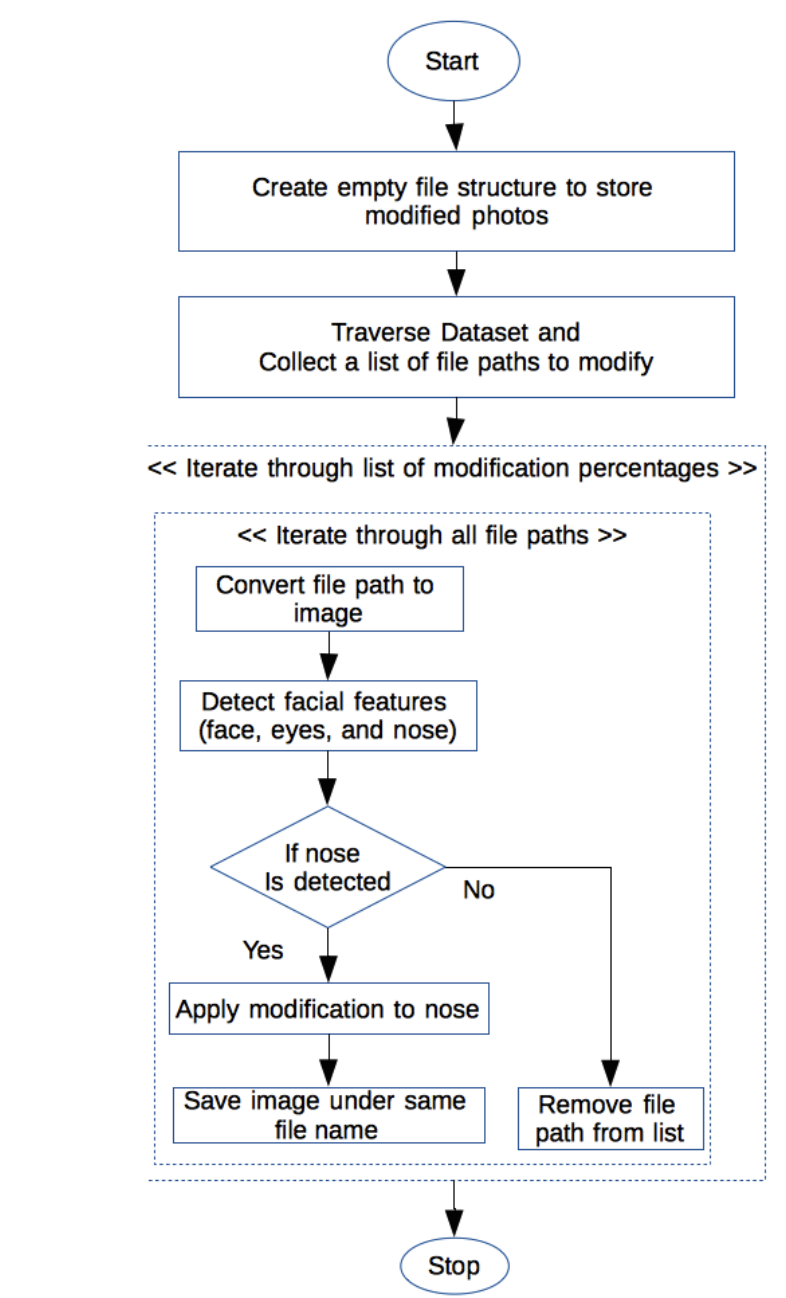

Figure 5: Process Diagram for generating a dataset full of simulated nose modifications

As Figure 5 shows, the first major step is to make sure an empty structure is created. The modified images can be filled into this structure as this process repeats. The next set of major steps that follow is to construct a set of nose-modified images. Since each file has a file path on the computer's filing system, a list of file paths will be maintained that will correspond with each image. This list allows for ease of batch processing or single image manipulations (list of one). To add in a layer of abstraction, a file system traversal class was constructed where the class has all of the details for extracting the desired file paths of the dataset. For the AT\&T dataset, each person's set of photos is grouped into a folder. All of the subject's folders are grouped in another folder together, as Figure 2 shows.

To aid in the generation and consistency of feature detection in the nose, the features of the face will be automatically detected and the coordinates of the nose. Although there are many automatic 
feature detection algorithms, like Active Shape Model, ASM [8], and the Haarcascade Classifier, the feature algorithm that was selected was the Haarcascade Classifier.

The Haarcascade is a quick and effective feature detector that allows for rectangle coordinates around a particular feature to be collected. Although there are other algorithms, like ASM [8], that were used in the other Facial Recognition Papers like Face-off [7], these algorithms are more accurate and computational intensive. Since no other research could be found that scientifically modifies the facial features as done herein, the more memory efficient and quicker feature detector was chosen for this experiment instead of the more memory intensive feature detector. Future work could use more powerful and elaborate feature detectors to explore the impact nose modifications impact feature detection.

If the Haarcascade Classifier cannot detect a person's nose (or face) then the modification for that particular image is not generated. It was not considered until after the experiment that one could allow for an interactive mode for noses that could not be detected by the algorithm. If an interactive mode were added to the experiment, a compromise to the consistency and control of a modification to the nose between iterations would exist.

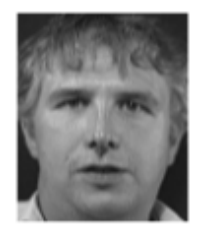

(a)

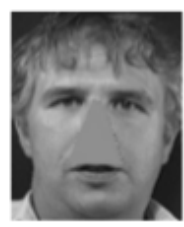

(d)

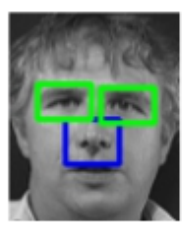

(b)

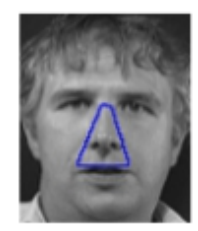

(c)

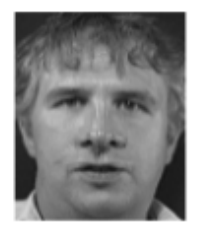

(e)

Figure 6: Example of how the facial features are detected and modified.

As an example of the process, a subject was selected from the dataset and some of the modifications were applied. As you can see in Figure 6a, the first image is of the original subject. Figure $6 \mathrm{~b}$ illustrates the features detected for this person, that is the detected eyes and nosedetected regions are shown. Modeled after the research procedure from Chou et al. [7] applied to simulate feature modification, a trapezoidal region around the nose is made. The trapezoid is determined by using the middle two points between the eyes, and the bottom of the detected nose rectangle. The trapezoid region is outlined in Figure 6c. This trapezoid is then used to isolate the nose, and apply the modifications to this region. To show the filtered out nose Figure $6 \mathrm{~d}$ is shown for illustrative purposes. Finally as Figure 6e shows the modified nose is applied back to the 
original image, yielding a modified image. A modification of $+50 \%$ growth to the width of the nose is shown in Figure 6e.

\subsubsection{Experiment}

The primary function of this tool is to run a trial of the experiment, or multiple trials if desired. Each trial that is run grabs both the original and modified photos (from generating the dataset in Section 3.3.1) and runs the experiment. After the trials are finished, a single file is created to store the results of the trials. For interoperability, the file is stored in the JSON file format. The libraries for reading in and writing to a JSON file are available in many general programming languages (e.g. Java, C, Lisp, Python, etc.).

When an Experimental Trial is being run, only one level of modification is considered in the trial. Each level of modification was generated with the Data Generation Tool. In terms of modifications, the original, unmodified, nose was said to be at $0 \%$. If the nose's width were doubled, then this would be $100 \%$ modification. Likewise for the nose to be shrunk in half, then this would be a modification of $-100 \%$. When running a trial, the original test images and the modified images (e.g. all modified images with a 100\% modification to the width of the nose) were used.

Since the main function of this tool is to run experiment trials, the process of the tool is to run an experiment trial or schedule multiple experiment trials to be run and collect the final results. The process can be visualized as such. 


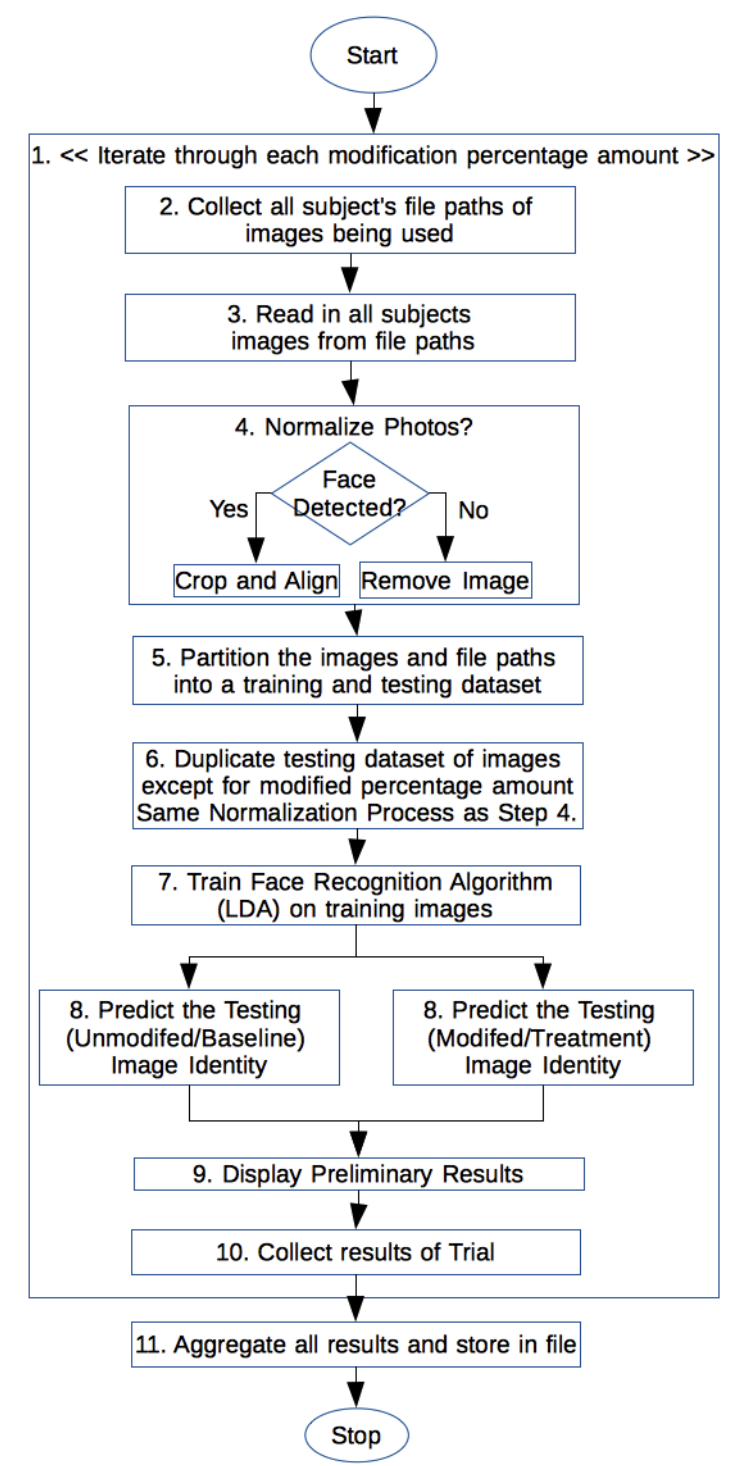

Figure 7: Process Diagram for running the experiment trial(s)

Figure 7 shows the process for setting up the experiment and running a trial is similar to the basic process described by Jain et al. [22]. Some differences to the basic Face Recognition procedures (Figure 1) can be reading in images and separating the images into the training and testing datasets for Face Recognition. The way that each experiment is executed is based on parallel lists. Each position in each list represents information about that person. For example, Figure 8 shows five lists. The first item in each list corresponds to a single person's image. Some of the information that is maintained throughout the trial is a string location on the hard drive of 1 . the person's image (both modified, and unmodified), 2. the original and modified photos of the person, and 3. the person's ground truth label (a unique number given to each person in the trial). 


\begin{tabular}{|c|}
\hline Original Filepath \\
\hline Modified Filepath \\
\hline $\begin{array}{c}\text { Ground Truth } \\
\text { Label }\end{array}$ \\
\hline Original Image \\
\hline Modified Image \\
\hline
\end{tabular}

\begin{tabular}{|c|c|c|}
\hline.$/$ dataset/s1/1.pgm &.$/$ dataset/s30/5.pgm & $\ldots$ \\
\hline.$/$ mod_dataset/-100\%width/s1/1.pgm &.$/$ mod_dataset/-100\%width/s30/5.pgm & $\ldots$ \\
\hline 9 & 23 & $\ldots$ \\
\hline s1 1.pgm original image array & s30 5.pgm Original Image Array & $\ldots$ \\
\hline s11.pgm modified image array & s30 5.pgm Modified Image Array & $\ldots$ \\
\hline
\end{tabular}

Figure 8: Parallel List Structure used while running experiment trials.

Although there are other ways to model the information in the lists for each person, the parallelism of the lists allowed for easy access and management of the information without adding the complexity of allocating additional memory for objects. Additionally this allows for non-Object Oriented Programs, such as Lisp, to be leveraged if so desired.

After a list of image locations is collected, the basic procedure for face recognition is followed, as proposed by Jain et al. [22]. With this procedure, step 4 of Figure 7 first looks to see if a face is detected in the image. If a face is detected, then the image will be normalized. Otherwise, that image will be removed from the trial. Normalization of each image, results in aligning the image so that the line between the eyes is horizontal, cropping the image to just the face, and normalizing each image to PGM (8-bytes of grey-scale intensity levels per pixel). Like with the Data Generation Step of this experiment, the algorithm used for detecting faces and facial features in an image is the Haarcascade Classifier.

Once all of the original images complete the preprocessing stage, the remaining images are partitioned into training (gallery) and testing (probe) sets. $40 \%$ of the images will be used for training and $60 \%$ of the images will be used for testing. This same split was applied in Singh et al. ([29] and [28]), Erdogmus et al. [9], and Kose et al. [18]. The 40/60\% split implies that at least one image of each person in the data is in the training set, and the training set images are not reused for the testing set.

From the testing set, another test set will be created from the original testing set called the "modified testing set". In the new "modified testing set", the same parallel list structure as the "original test set" is used. The only difference between these two test sets is the level of modifications to the nose. By creating these two sets, a comparison can be established between the original test set, and the modified test set.

Next the algorithms are trained on the training image set, and tries to "predict" who the person in the image is in each image of both the testing (probe) sets. These predictions are stored in yet another set of lists, parallel to the image lists. A prediction consists of both the label (unique number associated with each person in the trial) and the amount of distance that image is from the 
cluster of images for that particular person. If multiple predictions are grouped together for each image to the training (gallery) set, then ranks can be formed about each image. Ranks will be discussed in the next section in more detail.

Finally the predictions (results) and other information about the trial are stored, and the next trial is run. After all trials have been run, the results are aggregated and stored in a JSON file for the Experiment Analysis.

\subsubsection{Results (Experiment Analysis)}

The final tool that was created to examine the problem at hand is the Results/Experiment Analysis tool. Its primary function is to parse the file that was produced in the experiments, and provide a convenient model to access the results. For this tool, an object-based model was chosen to encapsulate the information about each image of each subject.

The results include predictions for each image in the test set that was created during the experiment trial(s), including both original (unmodified) and post-simulated nose plastic surgery (modified) images. These photos are identical except for the nose modification. Each prediction on an image has two parts, a predicted label and a predicted distance. The predicted label is a unique number associated with a particular person in the trial (this does not mean that the label, and the subjects number, sX, have to be the same) of the person that that test image is most similar to. When a test image's identity is being found, the most similar images are determined, and that is the predicted identity of the test image. The similarity of the image can be measured in terms of distance or confidence levels. When the gallery is set up, all of the training images are plotted in a multidimensional plane. Then, when a test image arrives, it is temporarily plotted and compared to all of the images in this training space. The total distance away from the each image is calculated. The closest distance to zero is desirable. The closest images (most similar) correspond to a subject label, which is returned as the predicted label and distance (identity and similarity) of the test image.

In most Face Recognition papers, Rank N performance metrics is talked about, which would be an extension of the most similar label/distance image in the gallery, also known as rank 1 . These ranks are accomplished by using the $\mathrm{k}$ Nearest Neighbor algorithm. The $\mathrm{k}$ Nearest Neighbor algorithm works by selecting the top k ranks (images/distances) and returned with each image. For the 10 nearest neighbors (rank 10), the top 10 most similar images are stored in a list, where the first element is rank 1 and most similar, the second element is rank 2 and the second most similar, etc.

A Picture Object is used to encapsulate the metadata and results of both the original and modified image. The results for the original and modified images are a list of ranked labels and distances for the guessed identity of the person in that image. A grouping of Pictures belongs to a Subject 
Object, which is a collection of Subjects make up a Trial Object. Each object is extracted from the results file, and has the basic process seen in Figure 9.

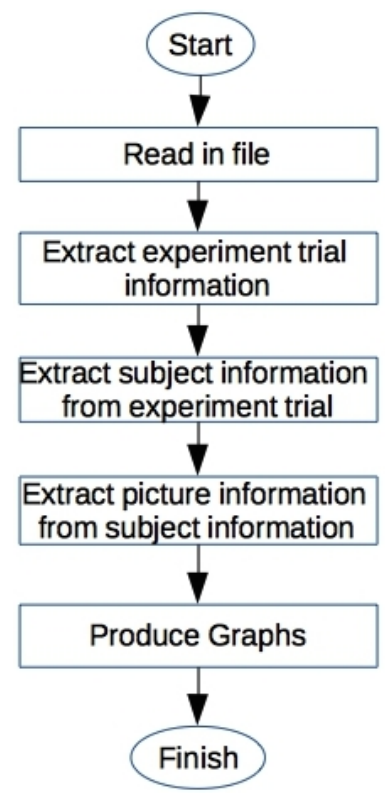

Figure 9: Process Diagram of how the Experiment Results Tool will import the experiment results file

As Figure 9 shows, the base object is the experiment trial, followed by a series of Subjects within each trial (still with the naming convention $\mathrm{SX}$, where $\mathrm{X}$ is the subjects number between 1 and 40). Finally each Subject in the Trial has a list of Pictures, which has the predicted labels/distances, and other information that can be viewed in a class diagram.

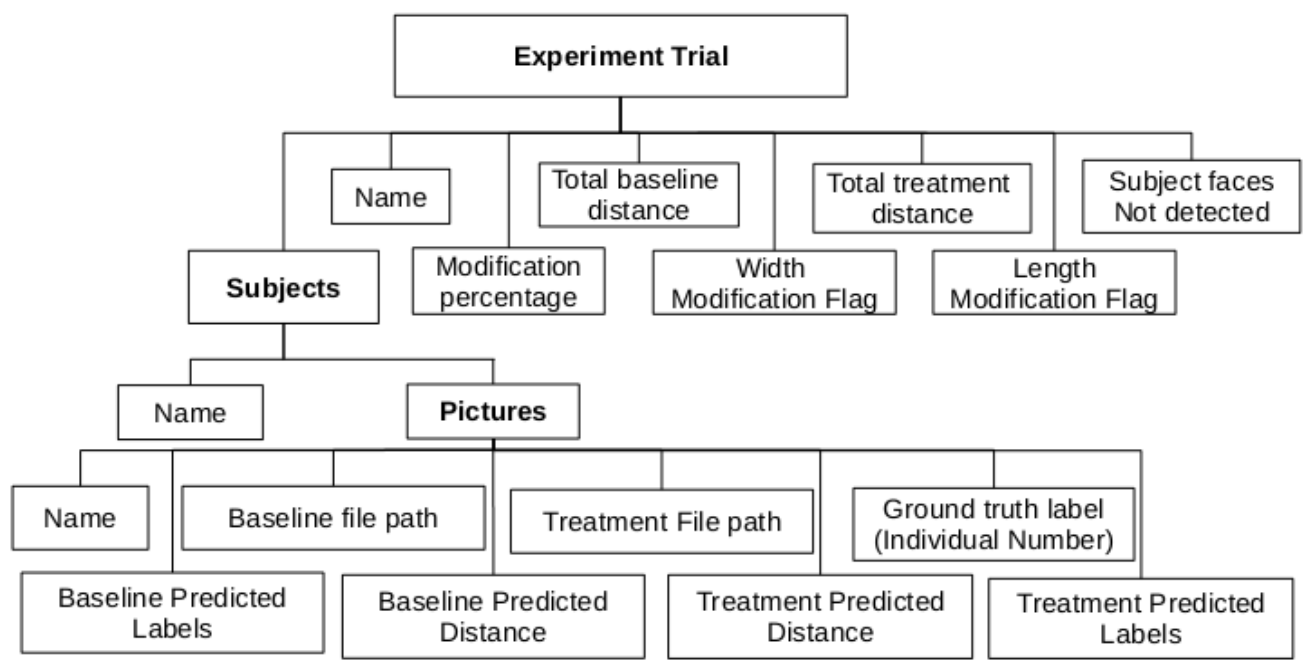

Figure 10: Class Diagram of the Experiment Results File 
As Figure 10 shows, other information besides the picture's information was stored. For example, information about the trial is stored such as if the width or length of the nose is being examined or the level of modification percentage the trial is considering. Although some values stored are for convenience, the total baseline predicted distance is just the summation of all rank 1 distances in the trial. The naming convention for the experiment results is a little different than the terminology for the last two tools. Based more on scientific terminology, the original photos go by the name of baseline, and the modified nose images are "treatments". Treatments refer to the changes after a process has been applied, which in this case is the simulated digital nose surgery on images. As Figure 10 also shows only a Picture Object contains both the baseline and treatment predicted labels and distances.

Figure 10 assumes that all of the images that have been recognized and had their identities predicted through the trained facial recognition algorithms. If an image was not detected, then it will not show up. For this reason, an additional list was stored in the Trial object that is of the "faces not detected". In the faces not detected list, a list of file paths, and labels are stored to allow for additional results to be gleaned from the results file.

Another assumption made in Figure 10 is that the training and testing sets are created through randomly selecting images from all possible images. For consistency, each trial had a single seed for the randomization for partitioning the dataset. This means that although the partition is random, the same partition will always be created between each trial since the seed is the same. This was chosen as a feasible route to allow tracking between photos for each trial. In this model, the same image can be looked at for all of the modifications of that particular image. To prevent selection bias of the training and testing images, a second set of experiments were run, that do not have the same randomization seed, allowing for a random split between. This second set of experiments will randomly split the images into training and testing sets for each modification increment. This reduces the chance that the selection of the training set has an impact on the results. Randomly splitting the training and testing set like this, no longer allows for the set of images being tracked like with the previous set of experiments.

With these assumptions, and the information from the experiment encapsulated in Objects, the next section 3.4, Evaluation of System, will talk about how the results of the system are analyzed.

\subsection{Evaluation of System}

With Figure 10's process, the objects are created but no tests for evaluation have been discussed. Since there is a baseline (the original) set of images, and a treatment (modified) set of images, a comparison between the two can be established. This is possible since the only variation between the treatment and baseline is the level of modification, the only uncontrolled variable is nose modification. A series of tests can be run on these results, including Closed-Set Identification Tests. 
Closed-Set Identification Tests are a special set of tests that focus on the available set of identities that the test (probe) images could be. For closed-set, it is known that the test image is of someone in the gallery, but it is up to the face recognition algorithm to determine who said person is. The alternative to Closed-Set is Open-Set Identification. In Open-Set Identification, the two-step process determines if the subject is known to the gallery and if so, what is the identity of the person in the image. A "best guess" for the identity will be guessed for the baseline and treatment sets since Closed-Set Identification assumes each testing image is of a known person in the training (gallery) set.

Additional tests that are used to evaluate the system do not associate with the Identity is the number of faces detected when creating the "modified" testing (probe) set and the use of Euclidean Distances to determine the guessed identities. These additional tests will only assist in the understanding of the Identity Tests.

\subsubsection{Randomization of the Test (Probe) Set}

For all of the Identity Tests, and the additional tests, one commonality between them all is reproducibility of setting the point where the partition is between the training and testing set. This will be applied once for way the Identity Test evaluates the system. For this work, two considerations were considered: a random partition is selected between trials of the experiment, and a set partition is repeated for each trial in the experiment.

The random partition selected for each trial means that the same images used in a trial will not be the same as the next trial. Using this type of partitioning decreases the chances of selection bias between trials of the experiment, but the impact of nose modification could not be monitored on a granular level for various photos in the experiment to see how the performance handles the modification.

When a set partition is repeated for successive runs of the experiment, the results will only be applicable for that set of images in the test set, not a full reflection of performance on the dataset. By using a particular example of partitioning, tracking of face recognition performance on a set of photos can be seen for different levels of nose modification.

Once the partition style is set, the original images are partitioned into training and testing sets. After this the testing set is duplicated to form a second test set except full of the corresponding modified image of original test set images. With this distinction, the randomly partitioned selection between trials will be called "intra-trial, randomly-selected test set" (Intra-Trial Randomization), and using only one partitioning for a set of trials will be called "inter-trial, randomly-selected testing set" (Inter-Trial Randomization). 


\subsubsection{Identity Tests}

One of the tests associated with Open- or Closed-Set Identification is the Cumulative Match Curve (CMC). The CMC works by graphing the percentage that the true identity of the test image is within the top $\mathrm{K}$ ranks. A CMC could be generated for both the baseline and the treatment images for each trial. This allows clarity and distinction between the baseline and treatment curves. A typical CMC graph has recognition values between 0.0 and 1.0 (or $0 \%$ and $100 \%$ ), which indicates the rate that the correct identity exists within the top $N$ ranks (guesses).

In order to glean understanding from all of the possible $\mathrm{CMC}$ graphs, numerical relationships between the baseline and the treatment curves are described as:

1. Baseline CMC curve is more accurate than the Treatment CMC Curve $(\mathrm{B}>\mathrm{T})$

2. Baseline $\mathrm{CMC}$ curve is less accurate than the Treatment $\mathrm{CMC}$ Curve $(\mathrm{B}<\mathrm{T})$

3. Baseline $\mathrm{CMC}$ curve is equal to the Treatment CMC Curve $(\mathrm{B}==\mathrm{T})$

4.

Once these numeric relationships are understood, each relationship is examined by looking at the recognition rates for all trials (baseline/treatment CMC curve pairs) within that category. One indicator of impact on the performance is examined by looking at the averages of the CMC curves start and finish. In this case, this would be considering Rank-1 rates against Rank-9 rates for both the baseline and treatment curves of each trial.

The Second Identity Test is to check the Image Similarity between the modified and original images and the respective Rank-1 labels (guesses). One such similarity check that can be used is the Peak Signal to Noise Ratio (PSNR). PSNR is an objective metric that has been used to compare the similarity of compression algorithms in terms of decibels (dB) [35]. For this work's purposes, the usage of PSNR will be used to show how similar the nose modification image compares with the original image. The more similar the two images are, the higher PSNR is. In the case of modifying the nose, only the modification differs between the original and modified image, so there should be expected some measurement above zero.

There are other measures that have been proven to more accurately show the difference between images, however, PSNR was selected for this project. It was available through OpenCV and could be used in a timely manner. Other measures would have pushed this project out of a realistic timeline. Previous research has shown that "one must be very cautious in interpreting its outcome" due to its limitations [5], [34], and [13]. However, PSNR still gives an indication of the difference between the modified and unmodified images, especially in conjunction with the CMC curve. 
One peculiar case arises when the same image is compared against itself. The PSNR value should be high, but the extent is not specified. There are two cases of PSNR values that will be considered:

a) Difference between two PSNR values (an optimal case and an original-modified case)

b) PSNR value of the original-modified image comparison only.

For both case (a) and (b) the original image and modified image is the cropped detected face, which is resized to resolution determined with the Resolution vs. Rank-1 Identity Test. For each case, two values will be considered, the average PSNR and the Median PSNR. These two values will show where the bulk of the values situate, and the non-outlier sensitive where the non-skewed middle value of the PSNR values is. Both types of PSNR graphs will be applied to both the Inter-Trial Random-Selection and Intra-Trial Random-Selection.

All of the graphs are plotted in 3-Dimensions in order to show how PSNR was impacted throughout all of the trials. The 3-Dimensions are PSNR, Modification Amount, and the Rank1 Recognition Rate. Within each of the graphs, four lines are plotted.

1. Average PSNR per Modification for the Baseline (unmodified) Rank-1 (R1) Rates

2. Average PSNR per Modification for the Treatment (modified) Rank-1 (R1) Rates

3. Median PSNR per Modification for the Baseline (unmodified) Rank-1 (R1) Rates

4. Median PSNR per Modification for the Treatment (modified) Rank-1 (R1) Rates

In the last Identification Test, Image Resolution vs. Rank-1 Recognition Scores is compared. This will examine the impact of image resolution (cropping, aligning, and resizing) has on Rank-1 Recognition Performance. As in the case with Image Similarity vs. Rank-1 Recognition, only the top predicted (guessed) label is considered in this test. Following Jain in the Basic Procedure of Face Recognition, Figure 1, all images are normalized before the person in the image is identified. After an image is cropped, the image of the face can be resized to any dimension. This test looks at the resolution of the detected and cropped faces. LDA depends on uniformity of resolution of images that it is comparing. Since the aligning and cropping allow for just the image of the face to be stored in the experiment, a series of resolution sizes is examined. When determining the ranges for image resolution, a square around the cropped face is extracted from the image, and then the resolutions are applied. For insight into the resolutions to test, Xu et al. [36] determined that usually higher resolution images help to improve the face recognition results, however there are other factors due to face recognition. For $\mathrm{Xu}$ et al. [36], they looked from a range of $8 \times 8$ up to $128 \times 128$ pixel images. Xu et al. [36] also found that $32 \times 32$ pixel images can be recognized by machines, but not by humans.

For this work, a range of 20x20 to 200x200 will be examined. Since a 32x32 pixel image was shown by Xu et al. [36] to be machine recognizable, a slightly smaller resolution was selected (20x20) and increments of 20 pixels in each dimension are considered. This range will overlap 
Xu et al. [36] and will increase past their initial testing. Since the images from the AT\&T dataset have a resolution of $92 \times 112$ pixels, enhancing the cropped and aligned face well past its original uncropped resolution limit seemed sufficient. The ideal resolution for rank-1 resolution is determined first and then used in all other Identity tests to reduce the number of graphs and considerations.

\subsubsection{Faces Not Detected}

The Faces that were not detected during the experiment were not identified face recognition, like CMC or Image Similarity Tests. It provides extra insight into the experiment on which images could be used in the testing set. In Section 3.3.2, the experiment goes through the basic face recognition steps described in Figure 1, such as Facial Detection, Normalization, Feature Extraction and Facial Identification. Facial Detection helps to provide a filter for the Facial Recognition System from non-face images. This is particularly useful, when the input images may have a face in them, so the Facial Recognition System only focuses on identifying faces that are detected.

One implementation of facial detection is the Haarcascade Classifier, where a series of particular features are precomputed and loaded into the detector. The detector then looks for a particular feature of the face, and will return coordinates around the feature of the detected face. The detection is run on a cascading series of rectangles looking at the particular feature. Some features that are provided from OpenCV out-of-the-box is the frontal face, side of the face, eyes, nose, mouth, etc. Unlike the computationally efficient Haarcascade, other implementations, such as ASM, uses a 3D Model of the face, and tries detecting the generic shape of the face. If the shape of the face can be detected then the 3D model is molded to the detected face for additional computation. ASM is more computationally intensive than the Haarcascade, but it is more robust to changes (such as expression, and posing/tilting of the face) and more power can be leveraged from it.

Since Facial Detection is the first step in Facial Recognition Systems, if a face is not detected then that image is not used in the experiment. From Section: 3.3.2, two parts use Facial Detection: collecting all images for partitioning into a training and testing set, and checking modified images for the duplicate modification testing set. When the images are initially being loaded in for the experiment, all images are run through Facial Detection. Following the basic facial recognition process, outlined in Figure 1, the face is extracted and cropped down to just the face in the image. The other time Facial Detection is used is when the modified dataset is being constructed. If the modified face is not detected, then the unmodified, corresponding, test image pair is also removed from the test set since it does not show any impact for face recognition. Since all of the images available in the AT\&T dataset are of peoples' faces, when an image is not detected, a person in the picture is not be identified by Facial Recognition. 


\subsubsection{Euclidean Distance}

The driving force behind identiciation in facial recognition algorithms when determining the identity of the test (probe) image is a distance metric. One such metric for determining the identity of the probe image is to find the closest most similar image to the test image. This work used a common similarity (distance) metric called the Euclidean Distance for determining the distance between two images. For example, when LDA is predicting the identity of the image, it first determines the discriminative information about the person's face, and plots that in a multi-dimensional space. The most similar image to the temporarily plotted test image is the "best-guess" for an identity. This process is repeated to get multiple ranks of guesses.

The Euclidean Distance measures the distance between two points. When a probe image is temporarily plotted in the algorithms testing space, the line between the test image and each image is calculated. The shortest line segment, distance, is the closest, most similar image. A distance of zero would mean that the same training image face was found that matched the test image exactly.

The distance measured between the training and test images act as a form of confidence of the guessed identity. The closer the test image is to zero the more confident the algorithms are of the identity of the person in the test image. If the image is further away, then there is a better chance that another image may be "closer" to the test image. 


\section{Chapter 4 : RESULTS}

The final chapter of this paper will discuss the results of the experiment, as outlined in Section 3.4.
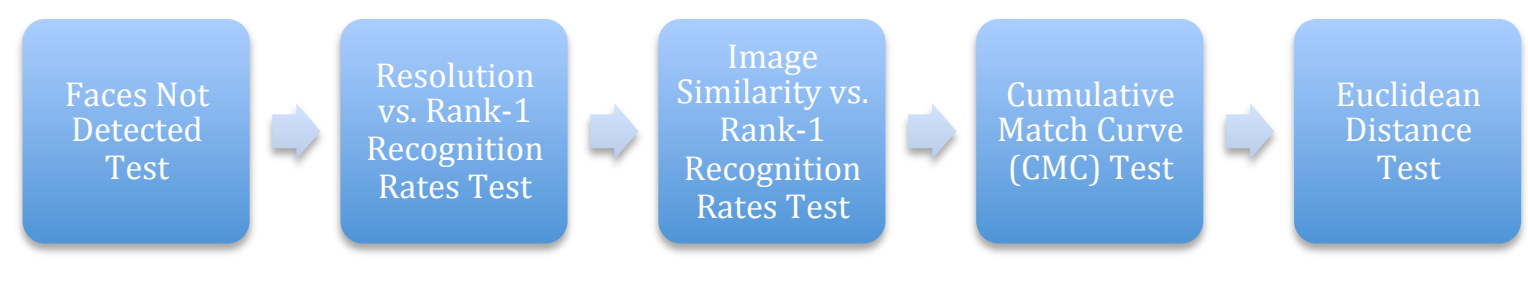

Figure 11: Visualization of the Results Section Organization

As Figure 11, the Faces Not Detected Test will be discussed first since it shows the test images removed from the test set for both Intra-Trial and Inter-Trial Randomization test sets as a results of the modified face not being detected. Section 4.2 describes the ideal resolution that will be used for the remaining Identity Tests. Section 4.3 illustrates the test of how similar the original and modified images are. Section 4.4 discusses the recognition rate as the number of included ranks is increased. Section 4.5 is an additional test that adds additional insight into the distances used for determining the Rank-1 labels. After the tests, Section 4.6 is used to tie the previous tests together and compare them to previous works. Section 4.7 and 4.8 is a summary of the results and conclusions. Finally Section 4.9, wraps up this work with suggestions for Future Work.

\subsection{Faces Not Detected During Trials}

As mentioned before, once an image has a detected face, it is then cropped down and resized to the specified resolution. Since resolution does not come into play until after the facial detection, this section will not be considering resolution size. Figure 12 and Figure 13 is of the second part from Section: 3.3.2. 

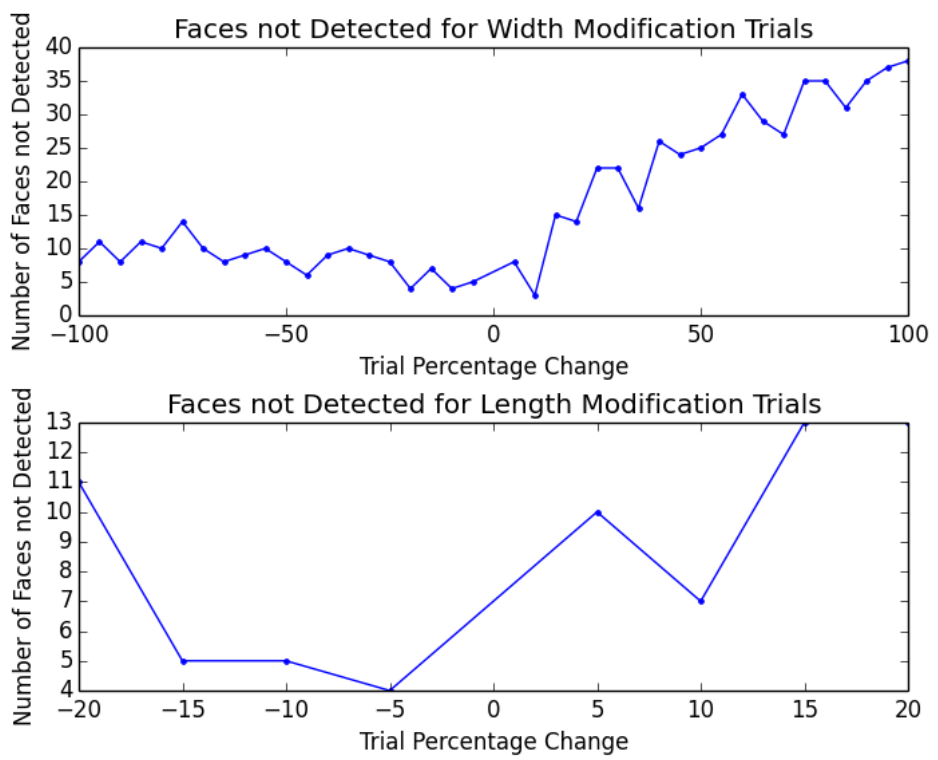

Figure 12: Faces Not Detected when loading the Intra-Trial Randomized images for the Experiment. Top graph is width modifications, and the bottom is length modifications.
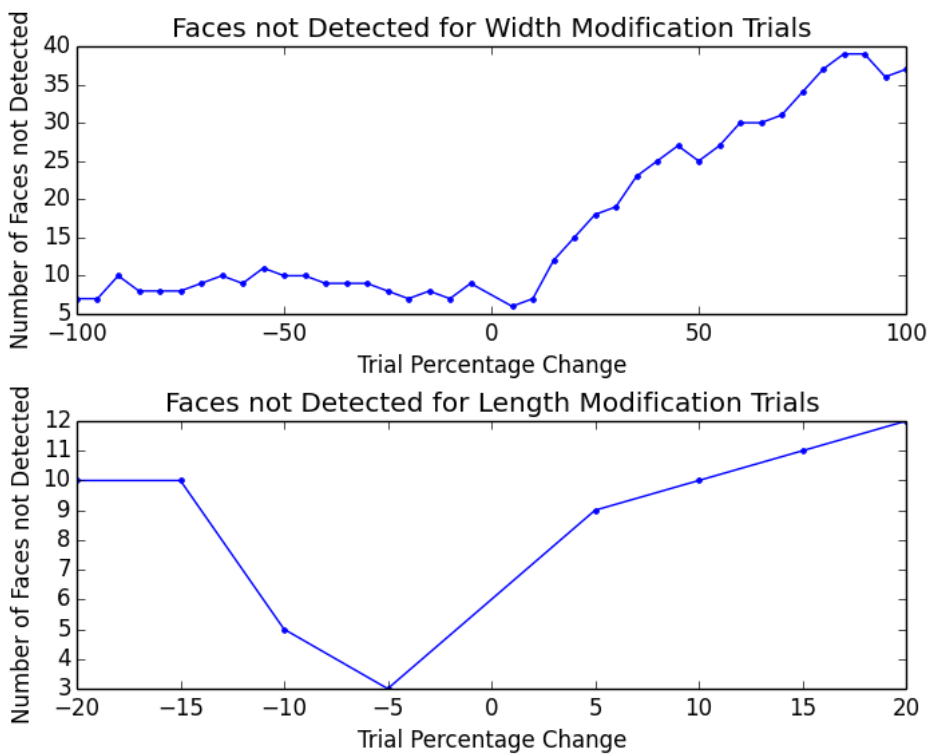

Figure 13: Faces Not Detected when loading in the Inter-Trial Randomized images for the Experiment. Top graph is width modifications, and the bottom is length modifications 
For both Figure 12 and Figure 13 shows, the top graph is of nose width modifications and the bottom graph is of nose length modifications. Figure 12 is of the Intra-Trial Randomizations, where the test images are randomly broken into two distinct training and testing sets. Figure 13 is of the Inter-Trial Randomization, where the same training/testing split is maintained for all of the trials. It is evident that Figure 12 and Figure 13 show similar trends both the width and length modification trials. Additionally the non-detected faces in Figure 12 are slightly higher than Figure 13 for shrinking the nose width but as the nose widens the same number of non-detected faces can be seen.

As Figure 13 shows, the top graph is of the Faces not Detected due to the Nose width modification. The lower graph focuses on the Faces not Detected due to the Nose length modification. As width modifications in Figure 13 go from $-100 \%$ to $0 \%$ (no modification), they stay between 5 and 10 detections. Once the nose goes from $0 \%$ to $100 \%$ (double the original size of the nose), the number of detection increases to about 40 non-detected faces. This high number of non-detected faces could make rank-1 scores more sensitive since there is less images in each trial. Similar trends can be seen for Figure 12.

On a far smaller scale, the nose length modification images had between 3 and 12 non-detected faces in Figure 13. The non-detected faces for the nose length modifications is can be read as, as the modification for the nose elongates or shrinks the harder it is for the Haarcascade Classifier to detect the face. Figure 12 shows similar trends for the nose length modification trials. Put specific percentages here. i.e. Modifying the length of the nose did not have a significant impact on facial detection. At most, about 5\% of the faces were not detected. Modifying the width of the nose had a more significant impact, at $75 \%$ increase, about $15 \%$ of faces were not detected. This shows that there is a threshold at which modifying the width of the nose makes the face undetectable. Since facial detection is the first step in recognition, this is certainly a negative impact on recognition.

\subsection{Resolution vs. Rank-1 Recognition Scores}

Intra-trial, randomly-selecting images refers to the experiment that splits all of the detected face images into $40 \%$ for training (gallery) and 60\% for testing (probe) sets. The split is fully randomized between each trial of the experiments. A trial is composed of a single set of unmodified (baseline) images and a single set of modified (treatment) images. To test the modified levels for the nose modifications, 48 independent trial runs are conducted (in 5\% intervals to the noses width and length). All of the resolutions are compared against the recognition rate for the trial, which is the rate that face recognition is able to correctly identify the person in the photo as its best guess (rank-1 label). In Figure 14, 40\% of the images are used for training and $60 \%$ are used for testing using Intra-Trial Randomization. 

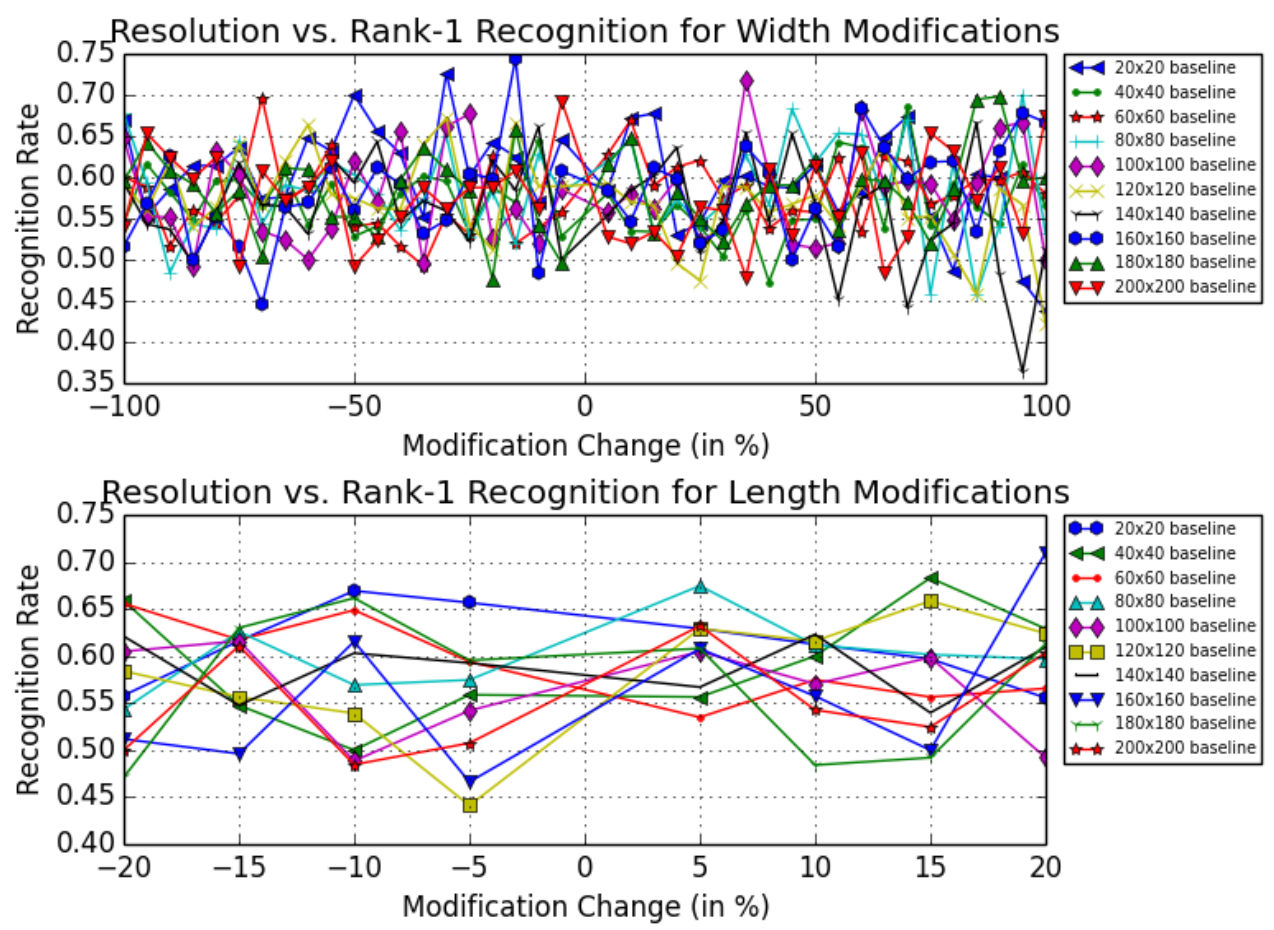

Figure 14: Intra-Trial Randomization, Resolution vs. Rank-1 Recognition Rates (top: Width \& bottom: Length).

As Figure 14 shows, the top graph is of the modifications to the width of the nose, and the bottom graph is to the length of the nose. In the width modifications of Figure 14 shows, the recognition rates for various recognition rates are very scattered. Some key features of the width modification graphs show that some of the higher resolutions, like 160x160, have higher recognition peaks but do not maintain a higher recognition rate for more modification trials. For some recognition rates, like 180x180 and 200×200, sit in the middle of the recognition rates and linger around a 0.56 recognition rate. Each Rank-1 Recognition Rate is enumerated for the nose width modifications in Table 8, which also shows these same conclusions. In the bottom graph, for nose length modifications, the amount of scatter is less, but no definitive ideal resolution. Some of the middle resolutions, such as 140x140, stay in the middle of the recognition rates, while not bouncing from the highest recognition rate to the lowest recognition rates. Table 9 lists the Rank-1 Recognition Rates for each resolution for each amount of modification to the nose length.

In comparison to using intra-trial randomization, inter-trial randomization splits all of the detected faces into $40 \%$ training (gallery) and $60 \%$ testing (probe) sets the same way between modification levels. Although selection bias could exist for this set, that is to say the images used to train on could factor into better results whereas intra-trial, randomly-selecting the training/testing split does not allow for that type of bias. An inherit property of inter-trial partitions is that a set of testing images can be tracked between trials. When the split (inter-trial randomization) is run 
against the resolution and measured by recognition rates, just like Figure 14, Figure 15 was produced.
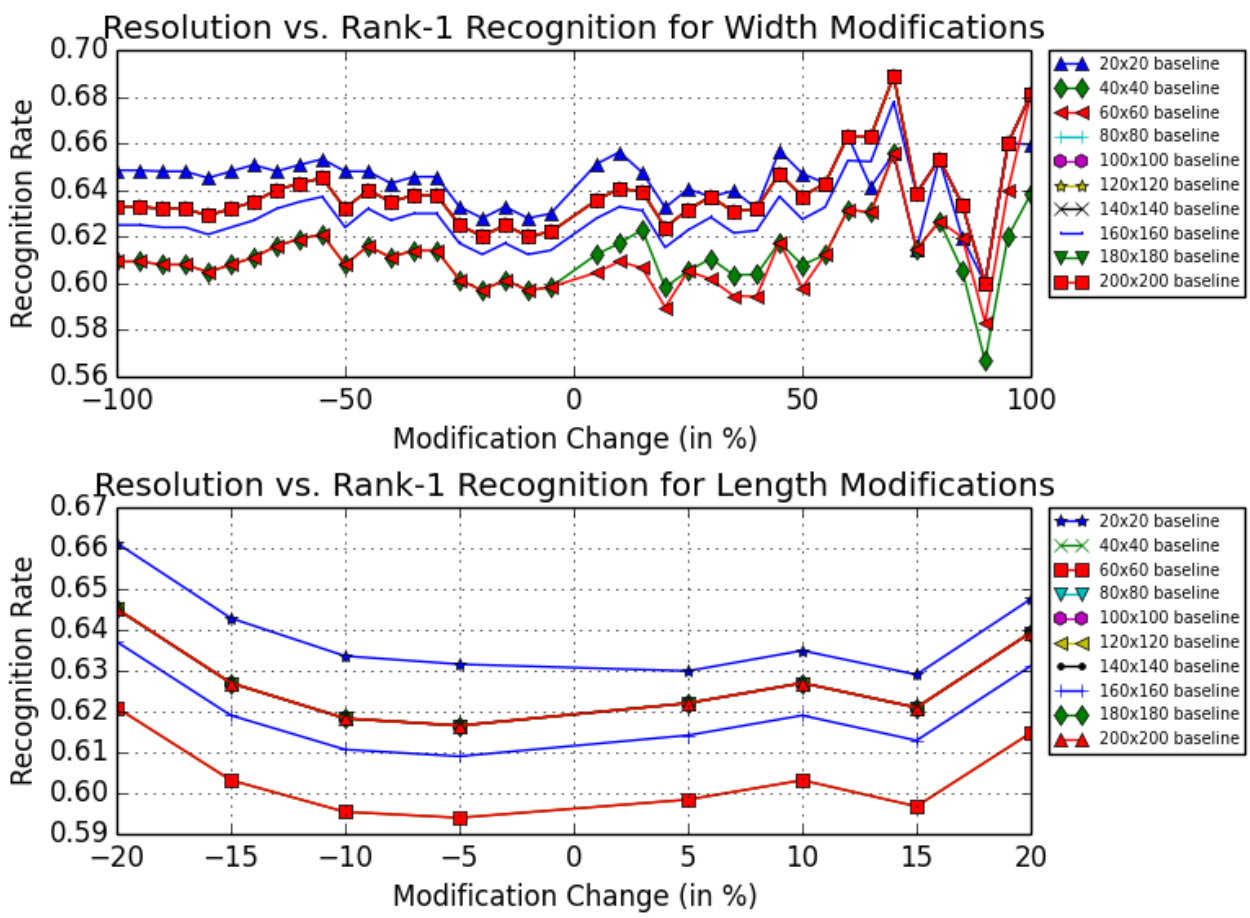

Figure 15: Inter-Trial Randomization, Resolution vs. Rank-1 Recognition Rates (top: Width \& bottom: Length)

In Figure 15, recognition rates seem to follow a common pattern as opposed to Figure 14. Just like with Figure 14, Figure 15 splits images in the same 40/60\% for training and testing. Figure 15 has two graphs, the top is for the modifications to the width of the nose, and the bottom graphs the modifications to the length of the nose. In Figure 15, fewer lines appear in the graph, which happens since the recognition rates for multiple resolutions overlapped. To illustrate this point, Table 10 and Table 11 in APPENDIX A shows the rank 1 recognition rates for each resolution for nose width and length modifications, respectively.

For both the width and the length trials in Figure 15, the highest recognition rate happens for a resolution of 20x20. The second highest recognition rate curve is $200 \times 200$, the highest resolution tested, which is only slightly lower than $20 \times 20$. As the modification gets larger for the width and length modifications, the gap between recognition rates for 20x20 and 200x200 decreases. For the width modifications, after a level of $+60 \%$ modification the $200 \times 200$ resolution line has a higher recognition rate than the $20 \times 20$ resolution line. 
When looking at Figure 14 and Figure 15, the same results follow [36], where higher resolution images are suggested. The higher resolution is both in the middle of Figure 14, as well as some of the highest recognition rates for Figure 15. This resolution will be used since the higher resolutions allow for higher dimensionality in the resized, cropped images, but are still human recognizable, as per Xu et al.'s [36] suggestion. For this reason, 200x200 resolution will be used for the remaining Identity Tests. It will also be used for all other algorithms for the sake of consistency.

\subsection{Image Similarity vs. Rank-1 Recognition Scores}

A table for each type of Randomization and Modification is provided in Table 24 of APPENDIX C. For conciseness and redundancy in the graphs, a few figures in this section are shown, but explanations for the remaining redundant graphs are also presented. All of the figures in this section have the same graph style, where the dotted line is the baseline recognition rate with the average PSNR in blue and the median PSNR in red. The solid line is the treatment recognition rate with the average PSNR in green and the median PSNR in green.

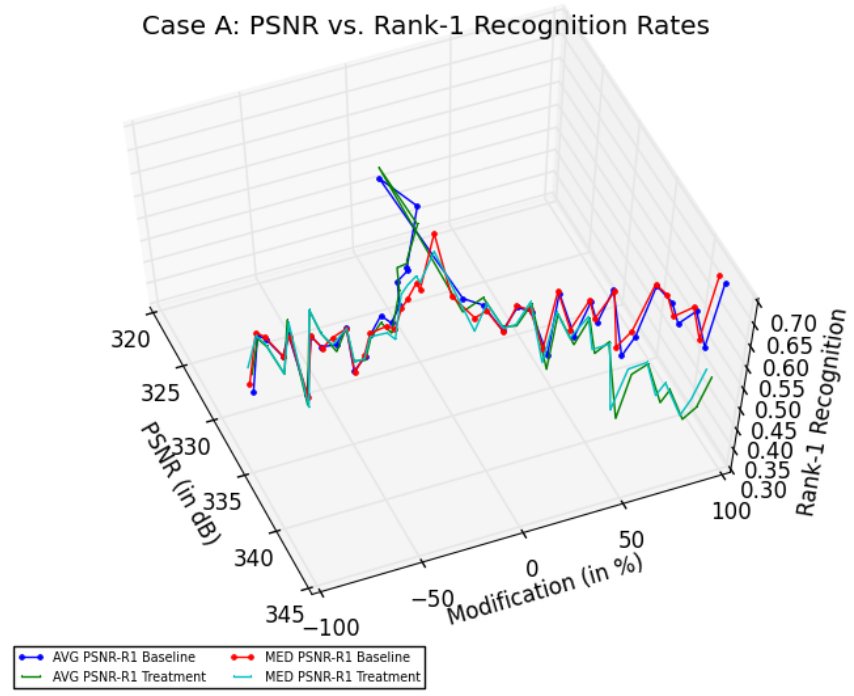

Figure 16: LDA Case (A) PSNR vs. Rank-1 Recognition Rates for IntraTrial Randomization for Width Modifications LDA

In Figure 16, the recognition axis is less prominently shown, however this shows the PSNR vs. Modification for the Intra-Trial Randomization Trials for Nose Width Modifications. As the modification amount grows from $-100 \%$ (half the nose's original width) to $-5 \%$, the PSNR value is 
consistently decreasing in similarity. As the modification amount moves from $-5 \%$ to $+5 \%$, a drop of $9 \mathrm{~dB}$ is observed for the average PSNR. For the same modification amount, the median is consistent. As the modification amount continues to increase from $+5 \%$ to $100 \%$ there is a steady rise in the similarity.

Case B: PSNR vs. Rank-1 Recognition Rates

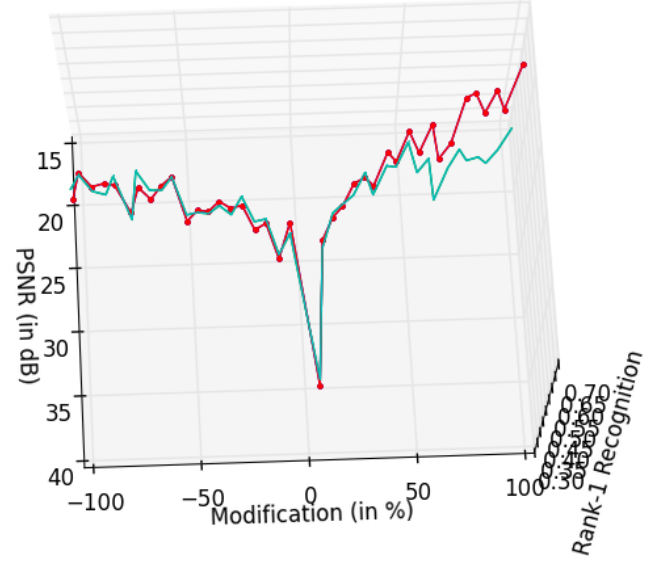

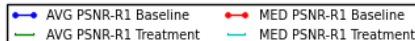

Figure 17: LDA Case (B) PSNR vs. Rank-1 Recognition Rates for IntraTrial Randomization for Width Modifications

Figure 17 plots just the similarity measurements between the original image and the modified image, as opposed to Figure 16. As the values in Figure 17 increase, the corresponding PSNR values in Figure 16 decrease. Unlike in Figure 16, Figure 17 only has two lines. However all four lines in Figure 17 are plotted but the baseline average and median mirror the treatment average and median lines. 
Case B: PSNR vs. Rank-1 Recognition Rates

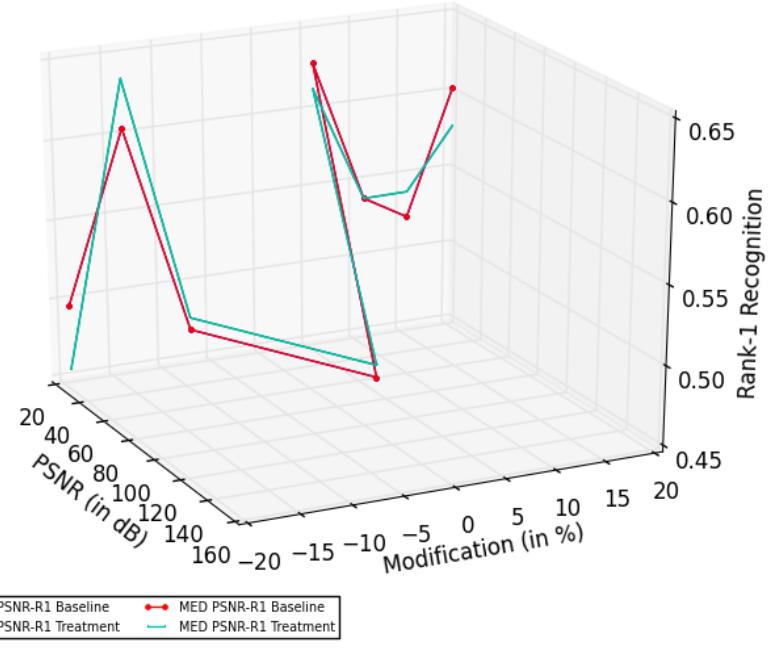

Figure 18: LDA Case (B) PSNR vs. Rank-1 Recognition Rates for IntraTrial Randomization for Length Modifications

Similar to Figure 17, Figure 18 has the same overlap in lines, but only differs in what type of nose modification is plotted. Figure 18 also has a lower recognition rate than that of Figure 16.

The redundant PSNR graphs were for the Inter-Trial Randomization nose width modification graphs, which had the same shape as seen Figure 16 and Figure 17 for the specific PSNR cases. The nose length modifications had a similar shape and PSNR values to Figure 18, and PSNR values are in the same range as in the Case (a) Intra-Trial Randomization nose width modifications (Figure 16). For all Inter-Trial Randomizations, a smoother curve was observed for each type of Inter-Trial Randomization as was shown in Figure 16, Figure 17, and Figure 18. As Figure 17 shows a complementary shape to Figure 16, so does Figure 18 have a complementary shape to its Case (a) equivalent graph. As with all of the figures in this section, similar figures and numbers were seen with the other facial recognition algorithms (PCA and LBPH) graphs.

As with all of the graphs in Section 4.3, no matter the level of modification, there is some similarity between the original image and the modified image. It is also seen that as the level of modification gets closer to no modification $(0 \%)$, the higher the similarity is between the two images. Even the minimal amount of modifications tested, $\pm 5 \%$ modification, has a similarity between the original and the modified image of $\sim 143 \mathrm{~dB}$ for the length of the nose, and $\sim 35 \mathrm{~dB}$ for the width. 


\subsection{Cumulative Match Curve (CMC)}

\subsubsection{CMC Graph Relationships}

Intra-Trial Randomization in the experiment refers to the method of selecting images for the training and testing set. When a new nose modification is considered, a new random partition between the training set and testing set is created as long as at least one image for each person is in each set, and there is no reuse of the same image in both sets.

When all of the CMC graphs were generated for this more randomized set of trials, the graphs were categorized as Section 3.4.2 suggested and compiled into Table 3

\begin{tabular}{|l|l|c|c|c|}
\hline Category & $\begin{array}{l}\text { Category } \\
\text { Description }\end{array}$ & $\begin{array}{l}\text { LDA } \\
\text { Frequency }\end{array}$ & $\begin{array}{l}\text { PCA } \\
\text { Frequency }\end{array}$ & $\begin{array}{l}\text { LBPH } \\
\text { Frequency }\end{array}$ \\
\hline 1. & $\begin{array}{l}\text { Baseline greater than } \\
\text { Treatment }\end{array}$ & 29 & 15 & 20 \\
\hline 2. & $\begin{array}{l}\text { Baseline less than } \\
\text { Treatment }\end{array}$ & 19 & 29 & 27 \\
\hline 3. & $\begin{array}{l}\text { Baseline equal to } \\
\text { Treatment }\end{array}$ & 0 & 4 & 48 \\
\hline Total & 48 & 48 & 1 \\
\hline
\end{tabular}

Table 3: Categorization of CMC Graphs by the CMC curve orientation for Intra-Trial, Randomly Selected Test Sets.

As Table 3 shows, category 1 shows 29 of the 48 CMC graphs had more accurate Baseline CMC curves than Treatment CMC curves. Category 1 can also be considered as nasal modifications have a negative impact on the Facial Recognition Performance. The remaining trials fell into category 2, where the treatment enhanced Facial Recognition Performance. Of the 29 category-1 graphs, 25 (of a possible 40) were width modification trials, and 4 (of a possible 8 ) where to length. Figure 19 is provided as an illustration of a typical Category 1 CMC curve. 
CMC Rate for 15\% Width Nose Modifications at Resolution 200x200, inter-trial randomly-selectec

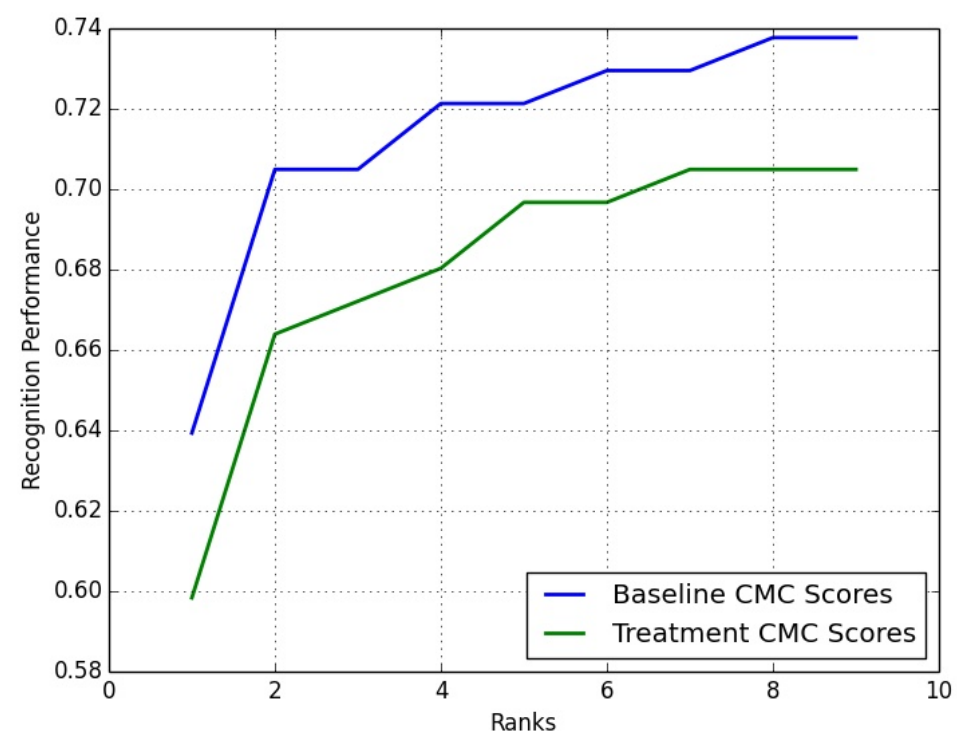

Figure 19: Example of a CMC graph where the Baseline (unmodified test images) CMC curve is greater than the Treatment (modified test images) CMC curve (also defined as Category 1).

As Figure 19 shows, the example is of $15 \%$ modification to the width. As can be seen in Figure 19, the Baseline Curve's recognition rate for each rank is more accurate on a rank-byrank basis. As more ranks are included, the baseline curve increases from a 0.64 rate to almost 0.74 , whereas the treatment increases from 0.60 to about 0.70 .

Out of the 19 Category 2 graphs, 15 were attributed to width modifications whereas 4 were to the length. A Category 2 graph would look similar to Figure 19, except the lines would be reversed. All of the categories when looked at for each nose modification level can be shown in Figure 20 and Figure 21. 


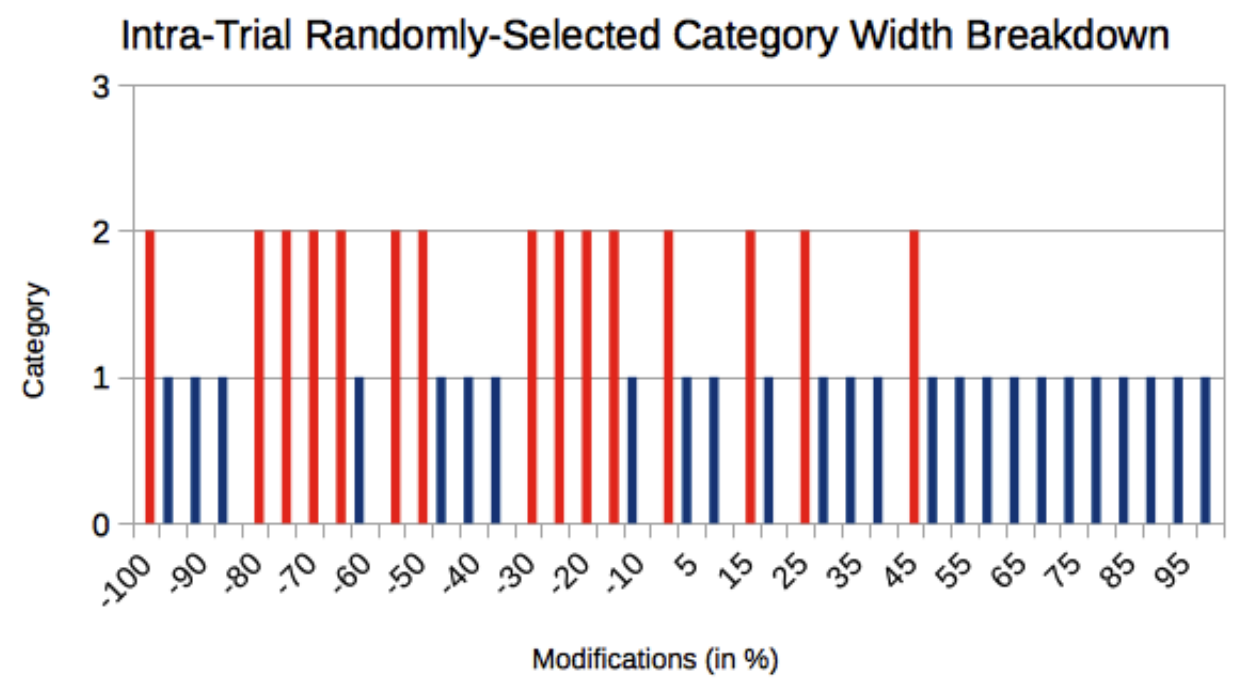

Figure 20: Distribution of which Width Modification falls into the CMC categories described above.

Intra-Trial Randomly-Selected Category Length Breakdown

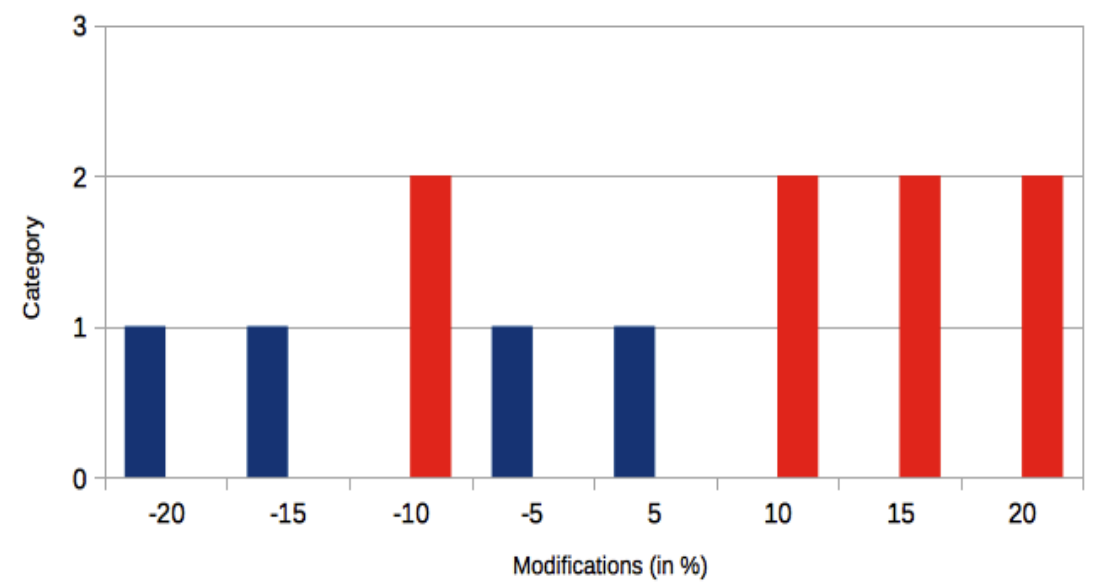

Figure 21: Distribution of which Length Modifications falls into the CMC categories described above.

Out of Figure 20 and Figure 21, five width modification trials, $-80 \%,-40 \%,-10 \%,-5 \%$, and $25 \%$, are close between Category 1 and Category 2 . In particular, trial $-80 \%,-5 \%$, and $25 \%$ 
where categorized as Category 2, but only by a little. For these particular trials, the Baseline CMC Values only differ from the Treatment CMC Values by $0.016,0.0079$, and 0.068 recognition rates, respectively. Similarly, $-40 \%$ and $-10 \%$ are categorized as Category 1 , but by a difference of 0.0079 , and 0.0079 in recognition rates, respectively.

As Table 3 and the distribution of CMC curves in categories, Figure 20 and Figure 21, indicate, the nose modifications have an impact on the recognition rate. The majority, 29 of the 48 (60.4\%), of the CMC results shows a decrease in Rank-1 Recognition performance indicated as Category 1 in Table 3. The remaining 39.6\% indicated that the nose modification had better recognition rates than the original, unmodified, image set. If the five close CMC graphs are classified as equal, then $54.2 \%$ belong to Category $1,35.4 \%$ belong to Category 2 , and the remaining $10.4 \%$ would be roughly no difference in Recognition Performance due to Nose Modifications.

Inter-Trial, Randomly-Selected Images in the experiment refers to the method of maintaining the same set of images for training and testing between trials but the initial partition is random. Similar to Intra-Trial Random-Selection, at least one image for each person is in the training and testing set, in which no image overlaps between the training and testing set.

\begin{tabular}{|l|l|l|l|l|}
\hline Category & $\begin{array}{l}\text { Category } \\
\text { Description }\end{array}$ & $\begin{array}{l}\text { LDA } \\
\text { Frequency }\end{array}$ & $\begin{array}{l}\text { PCA } \\
\text { Frequency }\end{array}$ & $\begin{array}{l}\text { LBPH } \\
\text { Frequency }\end{array}$ \\
\hline 1. & $\begin{array}{l}\text { Baseline greater than } \\
\text { Treatment }\end{array}$ & 38 & 31 & 25 \\
\hline 2. & $\begin{array}{l}\text { Baseline less than } \\
\text { Treatment }\end{array}$ & 10 & 11 & 19 \\
\hline 3. & $\begin{array}{l}\text { Baseline equal to } \\
\text { Treatment }\end{array}$ & 0 & 6 & 4 \\
\hline Total & 48 & 48 & 48 \\
\hline
\end{tabular}

Table 4: Categorization of CMC Graphs by the CMC curve orientation for Inter-Trial, Randomly Selected Test Sets.

As Table 4 shows, category 1 has more graphs than in the Intra-Trial CMC Graphs (Table 3). Since category 1 has more graphs, category 2 decreases in number since the total number of trials, 48, is consistent. An example of Inter-Trial CMC graphs is illustrated in Figure 22. 


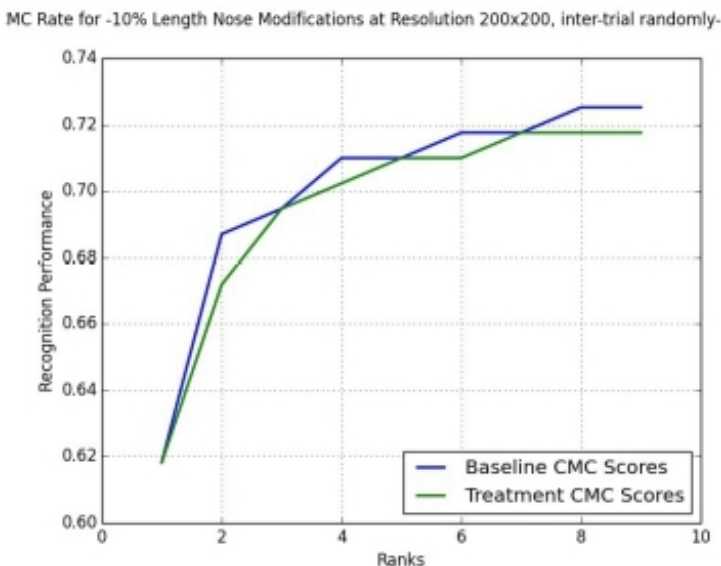

(a)

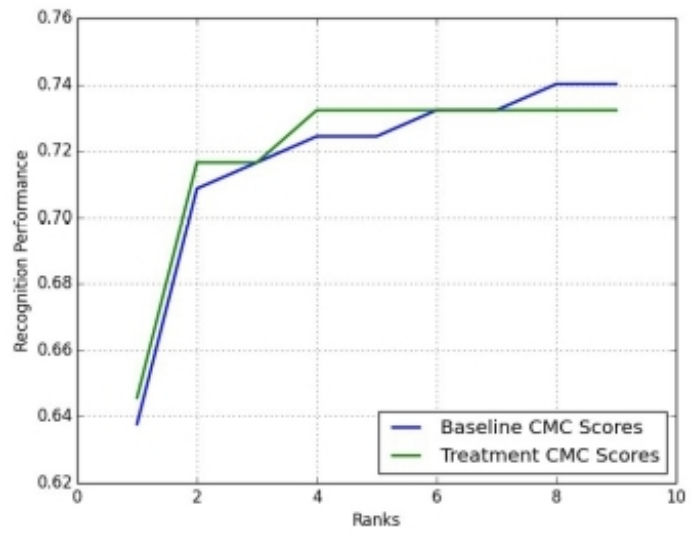

(b)

Figure 22: Examples of CMC Graphs for Inter-Trial, Randomly Selecting the test images. (a) Example of Baseline Curve greater than the Treatment (Category 1) (b) Treatment Curve is greater than the Baseline (Category 2).

Figure 22(a) shows an example of a Category $1 \mathrm{CMC}$ Graph, for a $-10 \%$ length modification to the nose. Figure 22(b) shows an example of a Category 2 CMC Graph, for $-35 \%$ width modification to the nose. Figure 22 is just two examples of the all 48 trials, so Figure 23 and Figure 24 shows which trials fell into each category for the Inter-Trial, Randomly Selected test images for the LDA trials.

Inter-Trial Randomly-Selected CMC Category Length Breakdown

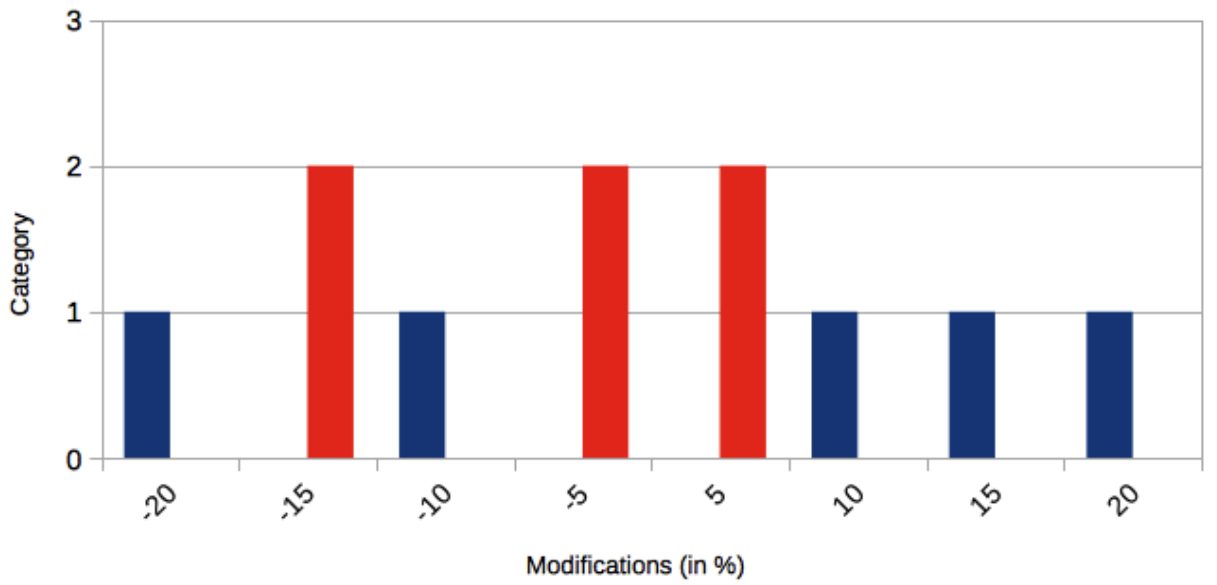

Figure 23: Distribution of which Length Modification falls into the CMC categories for Inter-Trial Randomization 
Inter-Trial Randomly-Selected CMC Category Width Breakdown

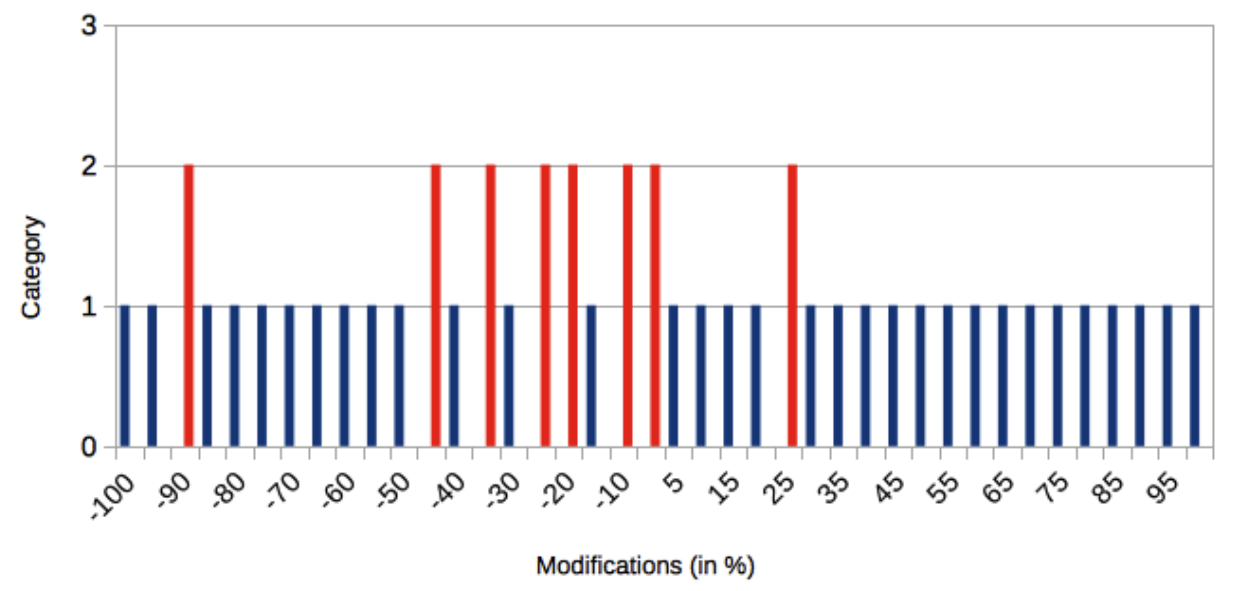

Figure 24: Distribution of which Width Modifications falls into the CMC categories for Inter-Trial Randomization

For both the Inter- (Figure 23 and Figure 24) and Intra-(Figure 20 and Figure 21) Trial Distribution of CMC categories, no graphs had baseline and treatment curves that were equal to one another. For Figure 23 and Figure 24, seven trials have close recognition rates between the Baseline and Treatment Curves. The trials that are classified as Category 1 but are close to Category 2 are $-95 \%$ and $-70 \%$ nose width modified trials, and $-10 \%$ nose length modified trials. The trials classified as Category 2 but are close to Category 1 are $-45 \%$ and $-10 \%$ nose width modified trials, and $-15 \%$ and $5 \%$ nose length modified trials.

As Table 4 lists and Figure 23 and Figure 24 show, the negative impact on Rank-1 Recognition Performance is increased when the same training/testing partition is used between trials. The majority, 38 of the 48 trials $(79.2 \%)$, of the CMC graphs show the same negative impact as is seen in the Intra-trial Randomly-Selected Results (Section 4.4.1). Similar results were found for the PCA and LBPH trials, so figures are not provided here. 


\subsubsection{Comparing CMC Categories}

\begin{tabular}{|l|l|l|l|l|}
\hline \multirow{2}{*}{ Category 1 } & \multicolumn{2}{|l|}{ Intra-Trial Randomly Selected } & \multicolumn{2}{l|}{ Inter-Trial Randomly Selected } \\
\cline { 2 - 5 } & $\begin{array}{l}\text { Baseline Rank } \\
\text { Value }\end{array}$ & $\begin{array}{l}\text { Treatment Rank } \\
\text { Value }\end{array}$ & $\begin{array}{l}\text { Baseline Rank } \\
\text { Value }\end{array}$ & $\begin{array}{l}\text { Treatment } \\
\text { Rank Value }\end{array}$ \\
\hline $\begin{array}{l}\text { LDA } \\
\text { Rank 1 }\end{array}$ & 0.5731 & 0.5095 & 0.639 & 0.5781 \\
\hline $\begin{array}{l}\text { LDA } \\
\text { Rank 9 }\end{array}$ & 0.715 & 0.6693 & 0.7398 & 0.7069 \\
\hline $\begin{array}{l}\text { PCA } \\
\text { Rank 1 }\end{array}$ & 0.5963 & 0.5508 & 0.5841 & 0.5878 \\
\hline $\begin{array}{l}\text { PCA } \\
\text { Rank 9 }\end{array}$ & 0.8014 & 0.777 & 0.804 & 0.7811 \\
\hline $\begin{array}{l}\text { LBPH } \\
\text { Rank 1 }\end{array}$ & 0.6741 & 0.5876 & 0.7153 & 0.6473 \\
\hline $\begin{array}{l}\text { LBPH } \\
\text { Rank 9 }\end{array}$ & 0.8128 & 0.7668 & 0.8187 & 0.7658 \\
\hline
\end{tabular}

Table 5: Comparison of CMC Rank Values between Intra- and Inter-Trial Randomly Selecting the Test Set where the Baseline CMC curve was greater than the Treatment CMC curve.

The average rates for LDA in Table 5 show an increase of 0.14 between the Rank- 1 and Rank9 baseline curves for Intra-Trial Randomization. A 0.16 increase is seen from Rank-1 to Rank9 for the treatment curve for Intra-Trial Randomization. For the corresponding baseline and treatment rates for Inter-Trial Randomly-Selecting the two sets show an increase of 0.10 and 0.13 for Rank-1 and Rank-9 rates. LBPH shows similar results, with a 9\% difference for Rank-1 and a 5\% difference for Rank-9.For PCA, there is an increase of 21\% from Rank 1 to Rank 9 for the Intra-Trial Randomization, and 22\% for Inter-Trial. Between the baseline and treatment rates there is difference of 0.05 Rank- 1 and 0.03 for Rank-9, showing that PCA was not affected by nose modification, while LBPH and LDA were negatively affected. As was previously mentioned, PCA has outperforms LDA when the number of training images is small. This may account for the higher overall rates of the PCA trials as well as the smaller impact made by modifying the nose.

Although a small difference, the Intra-Trial Results show a lower Rank-1 Rates for the Baseline and Treatment Curves while the Inter-Trial Rank-1 rates are higher. This is also true for Rank-9 scores as well. In the Intra-Trial Randomization, the difference in Rank-1 and Rank-9 rates had a slightly higher increase than is seen in the Inter-Trial Randomization. Numerically the difference between Rank-1 Baseline and Treatment curves shows Inter-Trial Randomization is 0.066 and 0.069 higher than the respective Intra-Trial Randomization. The 
same is seen for the Rank-9 scores, which are 0.025 and 0.038 higher for the Inter-Trial curves.

When the same information is compiled for the Category 2 curves, Table 6 shows the trials in which the treatment curves had a higher recognition rate over the baseline curve.

\begin{tabular}{|l|l|l|l|l|}
\hline \multirow{2}{*}{ Category 2 } & \multicolumn{2}{|l|}{ Intra-Trial Randomly Selected } & \multicolumn{2}{l|}{ Inter-Trial Randomly Selected } \\
\cline { 2 - 5 } & $\begin{array}{l}\text { Baseline Rank } \\
\text { Value }\end{array}$ & $\begin{array}{l}\text { Treatment Rank } \\
\text { Value }\end{array}$ & $\begin{array}{l}\text { Baseline Rank } \\
\text { Value }\end{array}$ & $\begin{array}{l}\text { Treatment } \\
\text { Rank Value }\end{array}$ \\
\hline $\begin{array}{l}\text { LDA } \\
\text { Rank 1 }\end{array}$ & 0.5668 & 0.5737 & 0.6263 & 0.6301 \\
\hline $\begin{array}{l}\text { LDA } \\
\text { Rank 9 }\end{array}$ & 0.7093 & 0.7258 & 0.7321 & 0.7328 \\
\hline $\begin{array}{l}\text { PCA } \\
\text { Rank 1 }\end{array}$ & 0.5863 & 0.5853 & 0.591 & 0.6039 \\
\hline $\begin{array}{l}\text { PCA } \\
\text { Rank 9 }\end{array}$ & 0.7806 & 0.7863 & 0.7997 & 0.7984 \\
\hline $\begin{array}{l}\text { LBPH } \\
\text { Rank 1 }\end{array}$ & 0.6768 & 0.6842 & 0.7223 & 0.7085 \\
\hline $\begin{array}{l}\text { LBPH } \\
\text { Rank 9 }\end{array}$ & 0.8053 & 0.8184 & 0.8136 & 0.8119 \\
\hline
\end{tabular}

Table 6: Comparison of CMC Rank Values between Intra- and Inter-Trial Randomly Selecting the Test Set where the Baseline CMC curve was less than the Treatment CMC curve.

For the Baseline Inter-Trial Randomly-Selected Results, an increase of 0.1058 is seen in Table 6 for LDA. The Intra-Trial Baseline rate has an increase of 0.1425 in its performance.

In comparison to the LDA Inter- and Intra-Trial Baseline average Rank-1 to Rank-9 rates, the Inter- and Intra-Trial Treatment average rates is 0.1027 and 0.1521 , respectively. This increase is almost the same amount as the Rank-1 and Rank-9 average rates for the baseline.

When comparing the baseline Rank-1 rates between Intra-trial and Inter-trial Randomization, the Inter-Trial is more accurate by 0.0595 over the Intra-Trial for LDA. Likewise to the baseline Rank-1 rates, the Inter-Trial Randomization rate for the treatments is 0.0564 more accurate than the Intra-Trial Randomization rates for LDA. 
Similarly to comparing the Rank-1 Results, the Inter-Trial baseline Rank-9 rate is more accurate to the Intra-Trial baseline Rank- 9 rates by 0.0228 . The Inter-trial treatment Rank- 9 rates are also more accurate by 0.007 over Intra-Trial Randomization. Similar in differences between the Rank-1 rates for both PCA and LBPH, the difference in the recognition rates are very close to one another.

As Table 5 and Table 6 shows, the Inter-Trial Random-Selected Curves are more accurate than the Intra-Trial Curves. The Baseline CMC curve's rate is higher for Rank 1 and Rank 9 rates in Table 5, and the Treatment CMC Curve's rate is higher than the Baseline Curve rates for Rank 1 and 9 rates in Table 6

When looking at the CMC ranks for the various algorithms (in APPENDIX B), the major decrease in performance occurs when the nose width increased by $40 \%$ or more.

\begin{tabular}{|l|l|l|l|l|}
\hline & \multicolumn{2}{|l|}{ Intra-Trial Randomization } & \multicolumn{2}{l|}{ Inter-Trial Randomization } \\
\cline { 2 - 5 } & Average & Median & Average & Median \\
\hline PCA Rank-1 & 0.0566 & 0.02632 & 0.0142 & 0.0141 \\
\hline PCA Rank-9 & 0.0183 & 0.0248 & 0.001 & 0.0 \\
\hline LDA Rank-1 & 0.1334 & 0.1451 & 0.1275 & 0.1356 \\
\hline LDA Rank-9 & 0.0752 & 0.660 & 0.050 & 0.0549 \\
\hline LBPH Rank-1 & 0.1232 & 0.0952 & 0.1060 & 0.1052 \\
\hline LBPH Rank-9 & 0.0639 & 0.0508 & 0.0639 & 0.0704 \\
\hline
\end{tabular}

Table 7: Average Difference between the Baseline and Treatment for $40 \%$ to $100 \%$ Nose Width Modifications

As Table 7 shows, the average difference between the baseline and treatment Rank-1 Recognition Rates for LDA and LBPH are between $10.6 \%$ and $13.3 \%$. PCA however is less than $5 \%$ different. The corresponding median differences show similar ranges.

When the differences between the baseline and treatment Rank-1 Recognition Rates for nose length modifications, the average difference were all under 3\%. For Rank-9 Recognition Rates, they were under $0.5 \%$. There is no point at which the modification levels exceeded a $10 \%$ difference between the baseline and treatment recognition rates. 


\subsection{Euclidean Distances and Confidence}

Similar to the format of previous sections, two types of Euclidean Distances will be considered with respect to the intra-trial and inter-trial randomization for partitioning the training/testing sets. As a quick reminder, intra-trial random partitioning exists when the training and testing images are partitioned randomly between trials (only one modification level of the nose is considered). Inter-trial random partitioning exists when a random partition is created for a series of trial runs and repeated between modification levels of nose.

When looking at the Euclidean Distances for Intra-Trial Randomization, Figure 25 plots the accumulated Euclidean Distances for all of the test images over all nose width modifications. Figure 25 's upper graph has two curves, a baseline and treatment curve. The baseline curve is the test images that have no modifications to the images. The treatment curve is the modified images that correspond to the baseline (unmodified) images. The lower graph in Figure 25 is the net difference between the baseline-accumulated trial distance from the treatment-accumulated trial distance. If the curve is above zero, then the baseline results have a higher accumulated distance, which would mean less confidence for the baseline test images, and if the curve is below zero the treatment has a higher accumulated distance (and would be less confident for the modified test images).

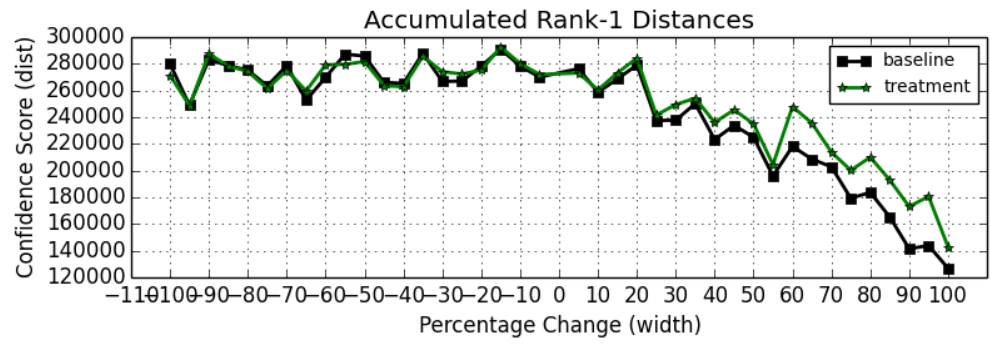

Figure 25: Intra-Trial Randomization Accumulated baseline and treatment distances for LDA

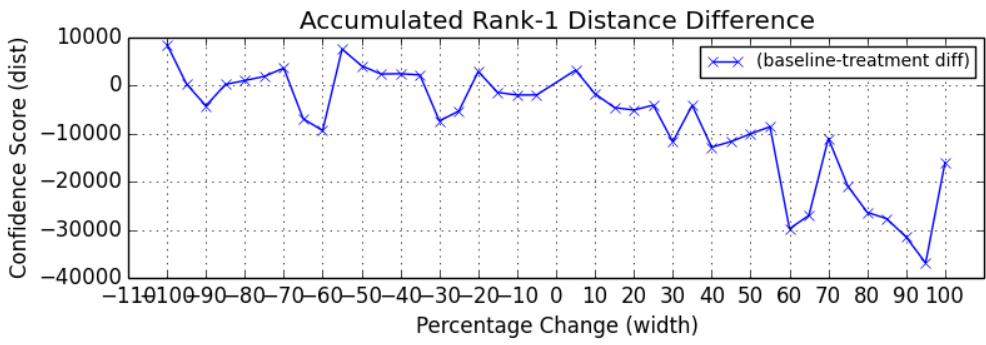

Figure 26: Intra-Trial Randomization Difference between Baseline and Treatment Accumulated Distances for LDA 
Figure 25 shows that as the nose modification enlarges, the Rank-1 identity distance grows. In Figure 25, when the treatment curve is above the baseline curve then the confidence in the identity of the modified photos decrease. As modifications increase the distance in the guessed identity, the potential for an incorrect identification of the person in the photo increases. In Figure 26, the difference between the baseline and treatment curves is shown for Figure 25.

Subtler than the Euclidean Distance for modification's to the nose's width, the nose length modification was also plotted in Figure 27. The format is the same as Figure 25.

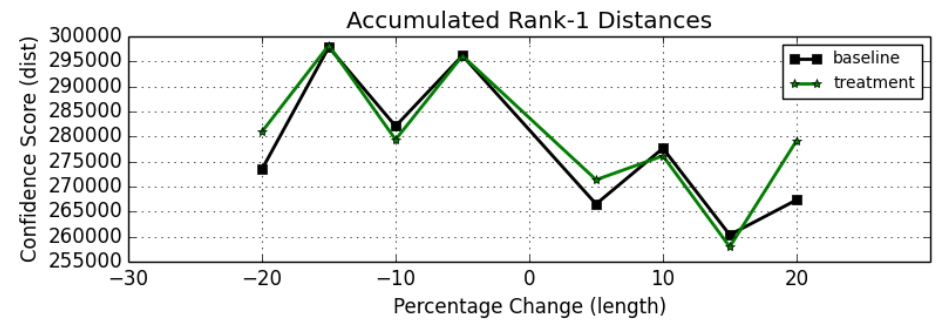

Figure 27: Intra-Trial Randomization Accumulated Baseline and Treatment Distances for LDA

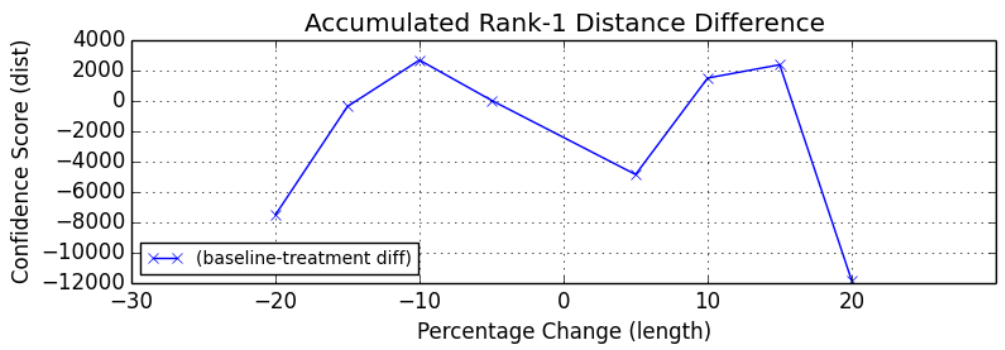

Figure 28: Intra-Trial Randomization Accumulated Difference between Baseline and Treatment Distances for LDA

As Figure 27 shows, the accumulated Euclidean Distances is smaller than that of distances in the width (Figure 25). A few trials, such as $-20 \%,+5 \%$, and $+20 \%$, all show that the distance for rank1 identities is much lower. The accumulated distances seen for both Figure 27 and Figure 25 are in the same ranges as well. As is also shown, for the trials $-20 \%, 5 \%$, and $+20 \%$, each has a higher treatment distance than the baseline, this would mean that the modified images in the test set was less confident in the identification. For the remaining trials, the trial scores are close to one another.

Unlike the random partitioning of the training/testing set between degrees of modification to the nose, Inter-Trial partitioning examined as well. In this the only change between trials is the 
modified images of the person's nose. In Figure 29, the accumulated distance is plotted against all width modifications.

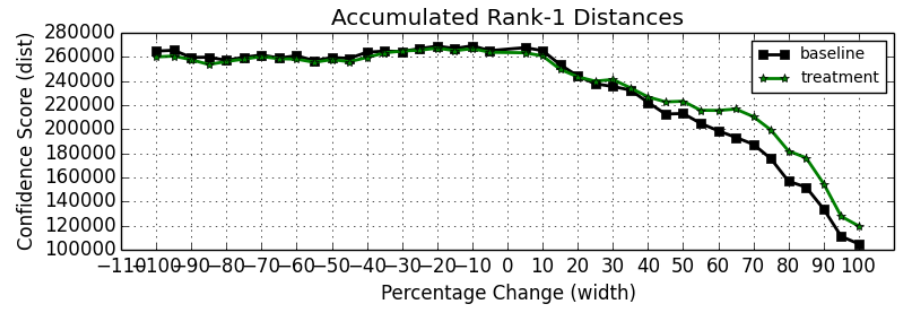

Figure 29: Inter-Trial Randomization for Accumulated Baseline and Treatment Distances for LDA

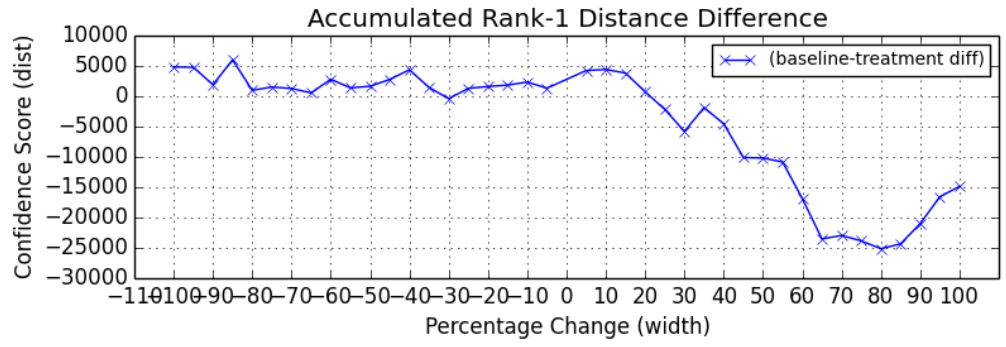

Figure 30: Inter-Trial Randomization Accumulated Difference between Baseline and Treatment Distances for LDA

Figure 29 shows that the distance curves are smoother than that of Figure 25. The same separation between the treatment (modified images) curve and the baseline (unmodified images) curve still increases when enlarging the nose. One difference between Inter-Trial width distance (Figure 29), and the Intra-Trial width distance (Figure 25) is that Figure 25 has a bigger gap as the modifications approach double the original size of the nose. Another is that Figure 25 has a higher accumulated distance as the nose widens. In other words, the Inter-Trial Randomization test images have closer distances than Intra-Trial Randomization, and is more "confident" in the guesses. Figures 29 and 30 show the same two graphs for the other algorithms. The same increase in the difference between the accumulated distance scores of the modified compared to the unmodified images exists, especially after the width of the nose is increased by $40 \%$ for LDA and LBPH. For PCA, the difference between the confidence scores is smaller but still visible. This could be due to some of the people in the training set had a small sample size. With a small sample size, previous research has shown that PCA has a higher chance to outperform LDA [23], [3], and [6].

In Figure 31, the Intra-Trial Randomization to nose length modifications is introduced. In the same format as the other graphs in this section, the upper graph shows the accumulated distances 
for the baseline (unmodified) images and the treatment (modified images), the lower is the difference between the baseline and treatment curves.

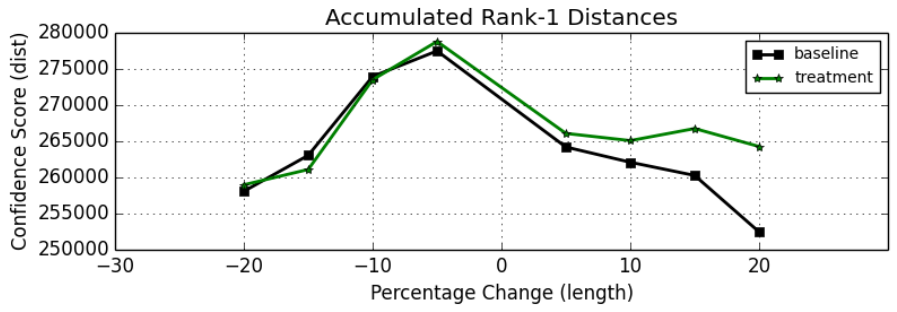

Figure 31: Inter-Trial Randomization Accumulated Baseline and Treatment Distances

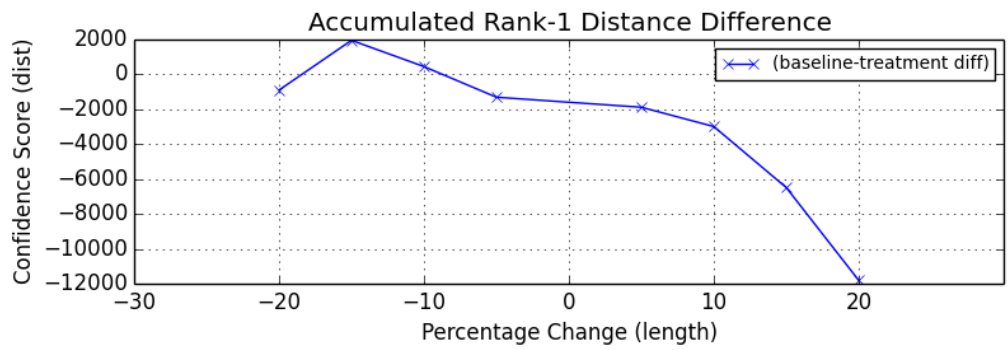

Figure 32: Inter-Trial Randomization Accumulated Differences between Baseline and Treatment Distances

Figure 31 shows that as the modification to the nose's length shrinks the accumulated distance in the images is close to the accumulated distance of the baseline. However when the nose's length is elongated, the accumulated distance between the modified and unmodified images increase. The distances in Figure 27 (Intra-Trial Randomization for nose length modifications) have bigger differences between the baseline and the treatment accumulated distances than in Figure 31. Figure 31 has more consistently higher accumulated distances for the nose modifications. Graphs of the results of the other two algorithms are in APPENDIX D. They are not shown here because they show the same insignificant difference in distance scores as the LDA trials. Changing the length of the nose does not appear to have a major effect on any of the algorithms run.

\subsection{Comparisons with Other Experiments}

This section discusses all of the results and ties together multiple sections and compares them to previous published works.

As Section 4.1 (Faces not Detected) shows, an image can be removed from the training/testing set before the training and testing set is created, and when the modified testing set is formed. The results from Section 4.1 show the removal of images from creating the modified testing set. A 
small number of faces were removed from the trials for the length modification and negative modification to the nose width, but a steady increase was observed for increasing the nose width.

The Haarcascade is a fast, and computationally efficient detector that relies on previous training of the detector to determine the particular feature. The standard Haarcascade training may not have been robust enough to detect the modified features.

Another possibility could be have been the nose modification images. Although the process follows Chou et al. [7] example, the implementation and usage of automatic detection is different. In this work, the nose is filtered like in Chou et al. [7]. In Chou et al. [7], the examples of detected features when using ASM was closer to the feature's edge than was observed for this work's detected feature edge with the Haarcascade. Once the feature is filtered, the nose is either stretched or compressed along the length or the width. One issue found is on some faces as the width of the nose is stretched, the region around the nose is stretched off the side of the face, as is illustrated in Figure 33.

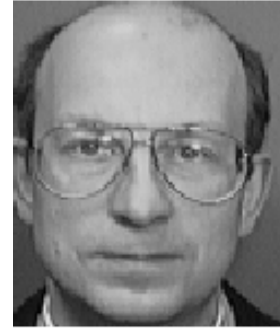

(a)

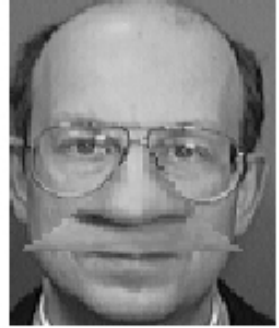

(b)

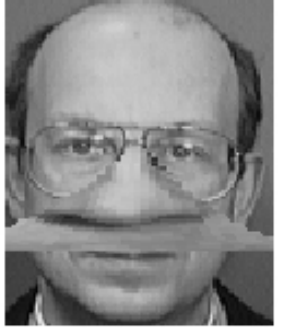

(c)

Figure 33: Examples of nose modification that extends beyond the width of the face. (a) original (b) $75 \%$ width modification (c) $100 \%$ width modification

As Figure 33 shows, the images of the faces in which the nose extended over the side of the face is for width modifications of $75 \%$ or greater. Because of the problems of nose modification in width, the length of the nose was restricted to $+20 \%$ so that it does not spill over the mouth. In spite of these problems with the generated nose modifications, the recognition rates of recognized and cropped down faces for recognition show a negative impact. Since the recognition is of cropped faces only, the images with double the size of the nose, which extends past the region of the face, will be limited to the detected face for recognition. Although the nose goes over the side of the face, cropping the detected faces to just the face helps manage the impact of this over-stretching.

Since non-detected faces are removed from the test before the Identity Tests can be run, these images do not directly impact the results. However the non-detected faces indirectly make the 
Identity Tests a little more sensitive to quicker changes in recognition rates and image similarity since there are less test images factoring into the results. If 40 is the maximum number of nondetected (greater than the maximum number of detected faces in Figure 12 and Figure 13), the testing set would have $83.3 \%$ (200 of the maximum 240) of the original test set images. Although the number of non-detected faces is at most 40, this number is small to report the remaining $83.3 \%$ of the remaining test images. Some of the spikes in Recognition Rate and Image Similarity in Section 4.3 and Section 4.4 could result from this slight sensitivity.

Although no previous work was found that assesses the impact of nose modifications in the way this work does, the work from Singh et al. [28] has CMC graphs and Rank-1 Recognition Rates. Although the process and the datasets differ from this work, Singh et al. [28] indicates that for their 192 subjects that have had real nose modifications, LDA has a drop of $\sim 24.1 \%$ in Rank-1 Identification Rate between their non-surgery based dataset and their plastic surgery based dataset.

Despite the number of subjects, Erdogmus et al. [9] pointed out a flaw in the process of Singh et al. [29], [28] in that the plastic surgery dataset only has a single pre- and post-surgery photo. Additionally Erdogmus et al. [9] state that the level on control in the pre- and post-surgery images to expression, lighting, make-up, etc. is not mentioned in Singh et al.'s work [28].

Erdogmus et al. [9] took a different approach than Singh. Similar to the process of Singh et al. [28], Erdogmus et al. [9] simulated the alterations and presented Rank-1 Recognition Rates and only used a single image of each person being matched for their gallery set. To increase the robustness of matching, an additional training set was used to help the Facial Recognition Algorithms determine the most distinctive facial attributes. Erdogmus et al. [9] reported that the Rank-1 Recognition Rate decreased by $\sim 10 \%$ for LDA when comparing the recognition rate for the original images in the test set and recognition rate when half of the test images of the person are modified, or $64.74 \%$ and $51.56 \%$, respectively.

Neither Erdogmus nor Singh mention the amount of nose modification for the subjects in their experiment, just that there is some level of modification. With the results indicated by Singh et al. [28], the closest level of difference in identification rates between pre- and post-modification is double the size of the original nose $(+100 \%)$. Unlike Singh, Erdogmus et al. [9] rate is closer to $+60 \%$ modification to the width of the nose with a max of $+100 \%$.

One major topic presented in each Identity Test section is with Inter-Trial and Intra-Trial Randomization. From the Identity Tests, the Intra-Trial Randomization (between each trial) the results are lower than that of the results from Inter-Trial Randomization. One precaution given for using Inter-Trial Randomization is that selection bias could be present in the results. In the IntraTrial Randomization, the results could be generalized to the results for the AT\&T dataset. The 
Inter-Trial Randomization is a specific instance of the Intra-Trial Randomization and only applies to that training/testing split.

\subsection{Summary}

This thesis focuses on determining the amount of impact that nose alterations have on LDA, PCA, LBPH. A framework is needed to test the impact on facial recognition. The framework that was constructed has three major components: a way to simulate nose modifications, a way to run experiments, and a way to read in and organize the post-experiment results in a meaningful way.

The framework for simulating nose modifications is based on the procedure used in by Chou et al. [7]. Chou et al. [7] used an automatic feature detector, ASM, to accurately identify facial features. Once the features were detected, the features of the person could be modified to that person's desire. Chou et al. [7] created their tool to assist in a patient wishing to undergo plastic surgery for particular features. When a patient wishes to modify their nose, a trapezoid formed between the eyes to the base of the nose was formed and manipulated. Other methods for manipulating the nose could have been pursued, such as the use of Thin Plate Spline (TPS) by Erdogmus et al. [9] on 3D models of the person's face, but were not for the sake of complexity. Although Chou et al. [7] has an inherent level of interaction on part of the user, this work is modeled after this.

The experiment execution was modeled vaguely off of Singh et al. [29], and Erdogmus et al. [9], but mostly from Figure 1. Through implementing the experiment component, two separate partitioning types were considered, one that was a specific example (Inter) that could show how a set of images impacted the facial recognition performance. The other (Intra) would be one that is more generalized to all situations for running on the dataset.

The final piece of the framework is a way to read in and interpret the results from the experiment. The results were stored in a JSON file, and read into an object-oriented based model for representing the data. Once read in, graphs were produced to show the results described in great detail in Chapter 4 as the Identity Tests, where the usage of recognition rate was heavily analyzed.

\subsection{Conclusions}

There is a level of similarity between the original and the modified image since the nose was the only region in the face that was changed. It was shown that nose modifications have a negative impact on automated facial recognition. Unlike other works, that lumped the nose modification (of any degree) together. When tested, this work quantitatively shows specific nose modification in pictures can frustrate automated Facial Recognition Algorithms. 
It was found that facial detection is negatively impacted by increasing nose width. Nose length modification did not have a significant impact, nor did decreasing the nose width. At 50\% increase in width, about 20-40 faces were not detected. In a small data set such as this one, that is a large negative impact.

There is very little impact on recognition when changing nose length by $20 \%$ in either direction. There is also very little impact when decreasing the width of the nose. There is, however, a negative impact when the width of the nose is increased. At about $40 \%$ increase in width, Rank-1 Recognition Rates reduced by about $10 \%$ in LDA and LBPH. For PCA, the average decrease in confidence was smaller, somewhere around 3\%. This could be due to the fact that with few training images, PCA outperforms LDA. LBPH focuses on texture, and the modified nose changes the texture of the face in the area around the nose. This change in texture is the reason LBPH confidence decreases when nose width is increased.

\subsection{Future Work}

Even though the impact of nose modification has been tested for LDA, PCA, and LBPH on the AT\&T dataset, this is only a single evaluation. Other face modifications, Facial Recognition algorithms, and Facial Recognition datasets need to be tested in order to expand the scope of these results from just AT\&T dataset to Facial Recognition Algorithms on multiple datasets. Other Facial Recognition Algorithms (both 2D and 3D Algorithms) can be used on the AT\&T dataset to explore the impact on Facial Recognition Algorithms. Other more robust and accurate feature detectors, i.e. ASM, etc., could detect the area around the nose more accurately, and would reduce the chance that the nose grows beyond the width of the face. Usage of other datasets, besides the AT\&T dataset, like the Face Recognition Grand Challenge (FRGC), Yale, or FERET datasets could be used to reduce the dependency upon a limited number of subjects, original image resolution, lighting, pose, and expression. Also, other measures of the accuracy of facial recognition could be used such as Mean Squared Error, Mean Absolute Error, or Mean Root Squared Error. The experiment could also be "flipped" so that the algorithms are trained on the modified images and then try to recognize the original face. Through exploring these changes in this work, nose modification's impact on Facial Recognition, as a whole, could be assessed even more accurately.

Besides changing the components of the experiment, one method not addressed was modifying both the width and the length of the nose modification at the same time. This change may achieve the level of dropping in LDA's performance as reported by Singh et al. [28]. The structure of the experiment can be changed to include multiple nose variations in one trial run, which would speed up the gathering of data from the experiment. Finally cross validation, the usage of all of the possible images of a person as part of the training set, could be incorporated so that selection bias can be reduced to nominal levels. These types of changes can be incorporated and expanded to add additional insight into how nose modification impacts LDA. 


\section{BIBLIOGRAPHY}

[1] T. Ahonen, A. Hadid, and M. Pietikainen. Face description with local binary patterns: Application to face recognition. IEEE Transactions on Pattern Analysis and Machine Intelligence, 28(12):2037, 2006.

[2] P. N. Belhumeur, J. P. Hespanha, and D. J. Kriegman. Eigenfaces vs. fisher-faces: recognition using class specific linear projection. IEEE Transactions on Pattern Analysis and Machine Intelligence, 19(7):711-720, 1997.

[3] Sushma N. Borade and Ramesh P. Adgaonkar. Comparative analysis of pca and lda. pages 203-206, 2011.

[4] T. Bourlai, J. Kittler, and K. Messer. Database size effects on performance on a smart card face verification system. pages 6 pp.-66. IEEE, 2006.

[5] Thirimachos Bourlai, Arun Ross, and Anil K. Jain. Restoring degraded face images: A case study in matching faxed, printed, and scanned photos. IEEE Transactions on Information Forensics and Security, 6(2):371-384, 2011.

[6] Lih-Heng Chan, Sh-Hussain Salleh, Chee-Ming Ting, and A. K. Ariff. Face identification and verification using pca and lda. volume 2, pages 1-6. IEEE, 2008.

[7] Jia-Kai Chou, Chuan-Kai Yang, and Sing-Dong Gong. Face-off: automatic alteration of facial features. Multimedia Tools and Applications, 56(3):569-596, 2012;2010;.

[8] T. F. Cootes, C. J. Taylor, D. H. Cooper, and J. Graham. Active shape models-their training and application. Computer Vision and Image Understanding, 61(1):38-59, 1995.

[9] N. Erdogmus, N. Kose, and J-L Dugelay. Impact analysis of nose alterations on $2 \mathrm{~d}$ and 3d face recognition. pages 354-359. IEEE, 2012.

[10] The American Society for Aesthetic Plastic Surgery. Cosmetic surgery national data bank statistics, March 2015. http://www.surgery.org/sites/default/files/2014-Stats.pdf

[11] Ralph Gross, Simon Baker, Iain Matthews, and Takeo Kanade. Face recognition across pose and illumination. In Stan Z. Li and Anil K. Jain, editors, Handbook of Face Recognition, pages 197-221. Springer London, 2011.

[12] J. D. Hunter. Matplotlib: A 2d graphics environment. Computing In Science \& Engineering, 9(3):90-95, 2007.

[13] Q. Huynh-Thu and M. Ghanbari. Scope of validity of psnr in image/video quality assessment. ELECTRONICS LETTERS, 44(13):800-U35, 2008.

[14] Itseez. The OpenCV Reference Manual, 2.4.9.0 edition, April 2014.

[15] Eric Jones, Travis Oliphant, Pearu Peterson, et al. SciPy: Open source scientific tools for Python, 2001-. [Online].

[16] Takeo Kanade. Picture processing system by computer complex and recognition of human faces. In doctoral dissertation, Kyoto University. November 1973.

[17] M. Kirby and L. Sirovich. Application of the karhunen-loeve procedure for the characterization of human faces. IEEE Transactions on Pattern Analysis and Machine Intelligence, 12(1):103-108, 1990.

[18] N. Kose, N. Erdogmus, and J. . Dugelay. Block based face recognition approach robust to nose alterations. pages 121-126. IEEE, 2012.

[19] Ton-Yee Lee, Yung-Nien Sum, Yung-Ching Lin, Leewen Lin, and Chungnan Lee. Three-dimensional facial model reconstruction and plastic surgery simulation. IEEE Transactions on Information Technology in Biomedicine, 3(3):214-220, 1999. 
[20] Tong-Yee Lee, Chao-Hung Lin, and Han-Ying Lin. Computer-aided prototype system for nose surgery. IEEE Transactions on Information Technology in Biomedicine, 5(4):271278, 2001.

[21] Jinshun Li and Yanyu Hu. Nose surgery simulation based on volumetric laplacian deformation. pages 1482-1487, 2011.

[22] S. Z. Li and Anil K. Jain. Handbook of face recognition. Springer, New York; London; 2nd;2;secondition; edition, 2011.

[23] A. M. Martinez and A. C. Kak. Pca versus lda. IEEE Transactions on Pattern Analysis and Machine Intelligence, 23(2):228-233, 2001.

[24] M. H. Ozkul and T. Ozkul. Computer simulation tool for rhinoplasty planning. Computers in Biology and Medicine, 34(8):697-718, 2004.

[25] Fabian Pedregosa, Gäel Varoquaux, Alexandre Gramfort, Vincent Michel, Bertrand Thirion, Olivier Grisel, Mathieu Blondel, Peter Prettenhofer, Ron Weiss, Vincent Dubourg, Jake Vanderplas, Alexandre Passos, David Cournapeau, Matthieu Brucher, Matthieu Perrot, and E' douard Duchesnay. Scikit-learn: Machine learning in python. $J$. Mach. Learn. Res., 12:2825-2830, November 2011.

[26] S. A. Rabi and P. Aarabi. Face fusion: An automatic method for virtual plastic surgery. pages 1-7. IEEE, 2006.

[27] F. S. Samaria and A. C. Harter. Parameterisation of a stochastic model for human face identification. pages 138-142, 1994.

[28] Richa Singh, Mayank Vatsa, Himanshu S. Bhatt, Samarth Bharadwaj, Afzel Noore, and Shahin S. Nooreyezdan. Plastic surgery: A new dimension to face recognition. IEEE Transactions on Information Forensics and Security, 5(3):441-448, 2010.

[29] Richa Singh, Mayank Vatsa, and Afzel Noore. Effect of plastic surgery on face recognition: A preliminary study. pages 72-77. IEEE, 2009.

[30] L. Sirovich and M. Kirby. Low-dimensional procedure for the characterization of human faces. Journal of the Optical Society of America. A, Optics and image science, 4(3):519524, 1987.

[31] Chiara Turati, Viola Macchi Cassia, Francesca Simion, and Irene Leo. Newborns' face recognition: Role of inner and outer facial features. Child Development, 77(2):297-311, 2006.

[32] Matthew Turk and Alex Pentland. Eigenfaces for recognition. Journal of Cognitive Neuroscience, 3(1):71-86, 1991. doi: 10.1162/jocn.1991.3.1.71.

[33] S. van der Walt, S.C. Colbert, and G. Varoquaux. The numpy array: A structure for efficient numerical computation. Computing in Science Engineering, 13(2):22-30, March 2011.

[34] Zhou Wang and A. C. Bovik. A universal image quality index. IEEE Signal Processing Letters, 9(3):81-84, 2002.

[35] Zhou Wang, A. C. Bovik, H. R. Sheikh, and E. P. Simoncelli. Image quality assessment: from error visibility to structural similarity. IEEE Transactions on Image Processing, 13(4):600-612, 2004.

[36] Xiang Xu, Wanquan Liu, and Ling Li. Face hallucination: How much it can improve face recognition. pages 93-98. Engineers Australia, 2013. 
[37] W. Zhao, R. Chellappa, and P. J. Phillips. Face recognition: A literature survey. $A C M$ Computing Surveys [H.W. Wilson - AST], 35(4):399, 2003. 


\section{APPENDICES}

\section{APPENDIX A : RESOLUTION VS. RANK-1 RECOGNITION RATES}

As Section 4.2 discusses, the chosen resolution is $200 \times 200$ pixels. This is determined by the graphs in Section 4.2, as well as the tables in this Appendix. A set of two tables will be presented showing the resolution vs. the modification.

\begin{tabular}{|c|c|c|c|c|c|c|c|c|c|c|}
\hline & $20 \times 20$ & $40 \times 40$ & $60 \times 60$ & $80 \times 80$ & $100 \times 100$ & $120 \times 120$ & $140 \times 140$ & $160 \times 160$ & $180 \times 180$ & $200 \times 200$ \\
\hline$-100 \%$ & 0.669355 & 0.544000 & 0.600000 & 0.677686 & 0.647541 & 0.593496 & 0.593496 & 0.515873 & 0.595238 & 0.539683 \\
\hline$-95 \%$ & 553846 & 616000 & 588710 & 593750 & 552846 & .559055 & 0.543307 & 0.568000 & 642857 & .653226 \\
\hline$-90 \%$ & 0.585366 & 0.581967 & 0.516129 & 0.483871 & 0.551181 & 0.619835 & 0.536585 & 0.626016 & 0.608333 & 0.624000 \\
\hline$-85 \%$ & 0.614173 & 0.548387 & 0.560976 & 0.543307 & 0.491803 & 0.539063 & 0.496063 & 0.500000 & 0.592000 & 0.598361 \\
\hline$-80 \%$ & 0.614173 & 0.595238 & 0.544715 & 0.537815 & 0.632813 & 0.563492 & 0.556452 & 0.552846 & 0.556452 & 0.624000 \\
\hline$-75 \%$ & 0.636364 & & 0.577236 & & & & 0.617886 & & 0.584000 & 0.491667 \\
\hline$-70 \%$ & 0.573770 & 0.565574 & 0.696721 & 0.543307 & & 0.565574 & 0.566929 & 0.445313 & 0.503937 & 0.608333 \\
\hline$-65 \%$ & 0.576000 & 0.606299 & 0.570313 & 0.590551 & & 0.619048 & 0.564516 & & & 0.572581 \\
\hline$-60 \%$ & 0.648438 & 0.542373 & 0.588710 & 0.584000 & 0.500000 & 0.664063 & 0.531250 & 0.569231 & 0.609375 & 0.588710 \\
\hline$-55 \%$ & & & & & & & & & 0.552000 & 0.619835 \\
\hline$-50 \%$ & 0.700000 & 28000 & 0.539683 & 0.603175 & 0.619048 & 0.574803 & 0.593750 & 0.560976 & 0.552000 & 0.491935 \\
\hline$-45 \%$ & 0.656000 & 0.540984 & 0.544715 & 0.580153 & 0.570313 & & 0.643411 & 0.611111 & 0.527559 & 0.523077 \\
\hline$-40 \%$ & 0.629032 & 0.579365 & 0.516129 & 0.536000 & 0.656000 & 0.560976 & 0.546875 & 0.592000 & 0.596899 & 0.551181 \\
\hline$-35 \%$ & 0.552000 & 0.601504 & 0.492308 & 0.572581 & 0.496000 & 0.640000 & 0.571429 & 0.531250 & 0.635659 & 0.587302 \\
\hline$-30 \%$ & 0.726563 & 0.595041 & 0.554688 & 0.650794 & 0.661290 & 0.671756 & 0.559055 & 0.548387 & 0.609375 & 0.562500 \\
\hline$-25 \%$ & 0.601563 & 0.532258 & 0.530769 & 0.527132 & 0.677165 & 0.543307 & 0.522388 & 0.604839 & 0.584000 & 0.587302 \\
\hline$-20 \%$ & 0.642857 & 0.587786 & 0.625954 & 0.582677 & 0.526718 & 0.512000 & 0.620155 & 0.598485 & 0.476923 & 0.587786 \\
\hline$-15 \%$ & 0.625000 & 0.569231 & 0.519380 & 0.519084 & 0.561538 & 0.666667 & 0.584615 & 0.744361 & 0.658915 & 0.608000 \\
\hline$-10 \%$ & 0.569231 & 0.643411 & 0.539063 & 0.628788 & 0.519380 & 0.589147 & 0.661417 & 0.484848 & 0.542636 & 0.562500 \\
\hline$-5 \%$ & 0.645669 & 0.528455 & 0.558140 & 0.581395 & 0.585366 & 0.589147 & 0.500000 & 0.607692 & 0.496063 & 0.692913 \\
\hline $5 \%$ & 0.584000 & 0.580645 & 0.627907 & 0.619048 & 0.558140 & 0.592308 & 0.553846 & 0.584000 & 0.616000 & 0.528000 \\
\hline $10 \%$ & 0.672000 & 0.534884 & 0.669355 & 0.645669 & 0.580153 & 0.570313 & 0.584615 & 0.546875 & 0.648438 & 0.519380 \\
\hline $15 \%$ & 0.677686 & 0.533898 & 0.589744 & 0.532787 & 0.561983 & 0.565574 & 0.601626 & 0.611570 & 0.532787 & 0.533898 \\
\hline $20 \%$ & 0.529915 & 0.566667 & 0.612613 & 0.573913 & 0.598214 & 0.495798 & 0.636364 & 0.598214 & 0.582609 & 0.504202 \\
\hline
\end{tabular}




\begin{tabular}{|c|c|c|c|c|c|c|c|c|c|c|}
\hline $25 \%$ & 0.544643 & 0.540541 & 0.620690 & 0.542373 & 0.521739 & 0.474576 & 0.508929 & 0.520661 & 0.550000 & 0.564103 \\
\hline $30 \%$ & 0.596491 & 0.504274 & 0.573913 & 0.567568 & 0.539130 & 0.589286 & 0.547009 & 0.535714 & 0.522523 & 0.559633 \\
\hline $35 \%$ & 0.601770 & 0.560345 & 0.590909 & 0.627273 & 0.718182 & 0.587719 & 0.654867 & 0.637168 & 0.567568 & 0.478992 \\
\hline $40 \%$ & 0.588785 & 0.472727 & 0.537736 & 0.566372 & 0.603774 & 0.557692 & 0.546296 & 0.605505 & 0.590476 & 0.609524 \\
\hline $45 \%$ & 0.587156 & 0.548077 & 0.560000 & 0.683168 & 0.518519 & 0.567308 & 0.653061 & 0.500000 & 0.590476 & 0.529412 \\
\hline $50 \%$ & 0.615385 & 0.549020 & 0.557692 & 0.617647 & 0.514019 & 0.578431 & 0.568627 & 0.561905 & 0.621359 & 0.613208 \\
\hline $55 \%$ & 0.557895 & 0.642857 & 0.623762 & 0.653465 & 0.520000 & 0.541667 & 0.451613 & 0.515789 & 0.536842 & 0.551020 \\
\hline $60 \%$ & 0.581633 & 0.636364 & 0.533333 & 0.652174 & 0.681319 & 0.588235 & 0.574257 & 0.684783 & 0.597938 & 0.630000 \\
\hline $65 \%$ & 0.648352 & 0.538462 & 0.626374 & 0.573171 & 0.590361 & 0.640449 & 0.590909 & 0.636364 & 0.595506 & 0.483516 \\
\hline$\overline{70 \%}$ & 0.673913 & 0.685393 & 0.619565 & 0.670588 & 0.595506 & 0.550562 & 0.441860 & 0.597826 & 0.569620 & 0.528090 \\
\hline $75 \%$ & 0.552941 & 0.541176 & 0.567901 & 0.457831 & 0.592593 & 0.552632 & 0.523810 & 0.618421 & 0.520548 & 0.653846 \\
\hline $80 \%$ & 0.486842 & 0.592105 & 0.578125 & 0.617647 & 0.547945 & 0.506494 & 0.544118 & 0.619718 & 0.585714 & 0.631579 \\
\hline $85 \%$ & 0.603175 & 0.564516 & 0.597015 & 0.457143 & 0.594203 & 0.458333 & 0.666667 & 0.534247 & 0.694444 & 0.571429 \\
\hline $90 \%$ & 0.600000 & 0.543860 & 0.596774 & 0.540984 & 0.660714 & 0.586207 & 0.482759 & 0.631579 & 0.698413 & 0.612903 \\
\hline $95 \%$ & 0.473684 & 0.615385 & 0.606557 & 0.700000 & 0.666667 & 0.566667 & 0.363636 & 0.678571 & 0.596154 & 0.532258 \\
\hline $100 \%$ & 0.437500 & 0.566038 & 0.580000 & 0.531915 & 0.500000 & 0.422222 & 0.511628 & 0.666667 & 0.600000 & 0.673913 \\
\hline
\end{tabular}

As Table 8 enumerates and Figure 14 top graph shows, there is no clear dominate resolution that the Rank-1 Rate is particularly consistent with. All of the tables in this section break down as the nose width modifications vertically and the Rank-1 Recognition Rate for each resolution listed horizontally. As an additional consideration, the resolution is of only the face in each image that is cropped and resized to the particular resolution.

\begin{tabular}{|r|c|c|c|c|c|c|c|c|c|c|}
\hline & $\mathbf{2 0 \times 2 0}$ & $\mathbf{4 0 \times 4 0}$ & $\mathbf{6 0 \times 6 0}$ & $\mathbf{8 0 \times 8 0}$ & $\mathbf{1 0 0 \times 1 0 0}$ & $\mathbf{1 2 0 \times 1 2 0}$ & $\mathbf{1 4 0 \times 1 4 0}$ & $\mathbf{1 6 0 \times 1 6 0}$ & $\mathbf{1 8 0 \times 1 8 0}$ & $\mathbf{2 0 0 \times 2 0 0}$ \\
\hline $\mathbf{- 2 0} \%$ & 0.558140 & 0.658730 & 0.655738 & 0.543307 & 0.604839 & 0.584000 & 0.620968 & 0.511811 & 0.471074 & 0.500000 \\
\hline$-\mathbf{1 5} \%$ & 0.616000 & 0.547619 & 0.617188 & 0.625954 & 0.616000 & 0.556452 & 0.547619 & 0.496124 & 0.629921 & 0.610687 \\
\hline$-\mathbf{1 0} \%$ & 0.669291 & 0.500000 & 0.648855 & 0.569231 & 0.488550 & 0.539063 & 0.603053 & 0.615385 & 0.661538 & 0.484615 \\
\hline $\mathbf{- 5} \%$ & 0.656716 & 0.558824 & 0.593985 & 0.574627 & 0.541985 & 0.441176 & 0.592593 & 0.466165 & 0.595420 & 0.507463 \\
\hline $\mathbf{5 \%}$ & 0.629032 & 0.556452 & 0.534884 & 0.674603 & 0.604839 & 0.629032 & 0.566929 & 0.607692 & 0.608000 & 0.632000 \\
\hline
\end{tabular}




\begin{tabular}{|c|c|c|c|c|c|c|c|c|c|c|}
\hline $10 \%$ & 0.612403 & 0.600000 & 0.574803 & 0.611570 & 0.570313 & 0.616000 & 0.622951 & 0.557377 & 0.484127 & 0.542636 \\
\hline $15 \%$ & 0.596899 & 0.682927 & 0.556452 & 0.601626 & 0.598425 & 0.658537 & 0.539683 & 0.500000 & 0.491935 & 0.524590 \\
\hline $20 \%$ & 0.555556 & 0.629032 & 0.565574 & 0.596774 & 0.492063 & 0.624000 & 0.609375 & 0.709677 & 0.612903 & 0.603306 \\
\hline
\end{tabular}

Just like Table 8, Table 9 shows the nose length modifications for the Intra-Trial Randomizations. Table 9 enumerates Figure 14 bottom graph.

Unlike Table 8 and Table 9, the specific case for randomly choosing the test images, Inter-trial Randomization, had slightly more parallel results. Similar to Table 8 and Table 9, there are overlapping recognition rates as shown in Table 10 and Table 11.

\begin{tabular}{|c|c|c|c|c|c|c|c|c|c|c|}
\hline & $20 \times 20$ & $40 \times 40$ & $60 \times 60$ & $80 \times 80$ & $100 \times 100$ & $120 \times 120$ & $140 \times 140$ & $160 \times 160$ & $180 \times 180$ & $200 \times 200$ \\
\hline$-100 \%$ & 0.648438 & 0.609375 & 0.609375 & 0.632813 & 0.632813 & 0.632813 & 0.632813 & 0.625000 & 0.632813 & 0.632813 \\
\hline$-95 \%$ & 0.648438 & 0.609375 & 0.609375 & 0.632813 & 0.632813 & 0.632813 & 0.632813 & 0.625000 & 0.632813 & 0.632813 \\
\hline$-90 \%$ & 0.648000 & 0.608000 & 0.608000 & 0.632000 & 0.632000 & 0.632000 & 0.632000 & 0.624000 & 0.632000 & 0.632000 \\
\hline$-85 \%$ & 0.648000 & 0.608000 & 0.608000 & 0.632000 & 0.632000 & 0.632000 & 0.632000 & 0.624000 & 0.632000 & 0.632000 \\
\hline$-80 \%$ & 0.645161 & 0.604839 & 0.604839 & 0.629032 & 0.629032 & 0.629032 & 0.629032 & 0.620968 & 0.629032 & 0.629032 \\
\hline$-75 \%$ & 0.648000 & 0.608000 & 0.608000 & 0.632000 & 0.632000 & 0.632000 & 0.632000 & 0.624000 & 0.632000 & 0.632000 \\
\hline$-70 \%$ & 0.650794 & 0.611111 & 0.611111 & 0.634921 & 0.634921 & 0.634921 & 0.634921 & 0.626984 & 0.634921 & 0.634921 \\
\hline$-65 \%$ & 0.648000 & 0.616000 & 0.616000 & 0.640000 & 0.640000 & 0.640000 & 0.640000 & 0.632000 & 0.640000 & 0.640000 \\
\hline$-60 \%$ & 0.650794 & 0.619048 & 0.619048 & 0.642857 & 0.642857 & 0.642857 & 0.642857 & 0.634921 & 0.642857 & 0.642857 \\
\hline$-55 \%$ & 0.653226 & 0.620968 & 0.620968 & 0.645161 & 0.645161 & 0.645161 & 0.645161 & 0.637097 & 0.645161 & 0.645161 \\
\hline$-50 \%$ & 0.648000 & 0.608000 & 0.608000 & 0.632000 & 0.632000 & 0.632000 & 0.632000 & 0.624000 & 0.632000 & 0.632000 \\
\hline$-45 \%$ & 0.648000 & 0.616000 & 0.616000 & 0.640000 & 0.640000 & 0.640000 & 0.640000 & 0.632000 & 0.640000 & 0.640000 \\
\hline$-40 \%$ & 0.642857 & 0.611111 & 0.611111 & 0.634921 & 0.634921 & 0.634921 & 0.634921 & 0.626984 & 0.634921 & 0.634921 \\
\hline$-35 \%$ & 0.645669 & 0.614173 & 0.614173 & 0.637795 & 0.637795 & 0.637795 & 0.637795 & 0.629921 & 0.637795 & 0.637795 \\
\hline$-30 \%$ & 0.645669 & 0.614173 & 0.614173 & 0.637795 & 0.637795 & 0.637795 & 0.637795 & 0.629921 & 0.637795 & 0.637795 \\
\hline$-25 \%$ & 0.632813 & 0.601563 & 0.601563 & 0.625000 & 0.625000 & 0.625000 & 0.625000 & 0.617188 & 0.625000 & 0.625000 \\
\hline$-20 \%$ & 0.627907 & 0.596899 & 0.596899 & 0.620155 & 0.620155 & 0.620155 & 0.620155 & 0.612403 & 0.620155 & 0.620155 \\
\hline$-15 \%$ & 0.632813 & 0.601563 & 0.601563 & 0.625000 & 0.625000 & 0.625000 & 0.625000 & 0.617188 & 0.625000 & 0.625000 \\
\hline$-10 \%$ & 0.627907 & 0.596899 & 0.596899 & 0.620155 & 0.620155 & 0.620155 & 0.620155 & 0.612403 & 0.620155 & 0.620155 \\
\hline
\end{tabular}




\begin{tabular}{|c|c|c|c|c|c|c|c|c|c|c|}
\hline$-5 \%$ & 0.629921 & 0.598425 & 0.598425 & 0.622047 & 0.622047 & 0.622047 & 0.622047 & 0.614173 & 0.622047 & 0.622047 \\
\hline $5 \%$ & 0.651163 & 0.612403 & 0.604651 & 0.635659 & 0.635659 & 0.635659 & 0.635659 & 0.627907 & 0.635659 & 0.635659 \\
\hline $10 \%$ & 0.656250 & 0.617188 & 0.609375 & 0.640625 & 0.640625 & 0.640625 & 0.640625 & 0.632813 & 0.640625 & 0.640625 \\
\hline $15 \%$ & 0.647541 & 0.622951 & 0.606557 & 0.639344 & 0.639344 & 0.639344 & 0.639344 & 0.631148 & 0.639344 & 0.639344 \\
\hline $20 \%$ & 0.632479 & 0.598291 & 0.589744 & 0.623932 & 0.623932 & 0.623932 & 0.623932 & 0.615385 & 0.623932 & 0.623932 \\
\hline $25 \%$ & 0.640351 & 0.605263 & 0.605263 & 0.631579 & 0.631579 & 0.631579 & 0.631579 & 0.622807 & 0.631579 & 0.631579 \\
\hline $30 \%$ & 0.637168 & 0.610619 & 0.601770 & 0.637168 & 0.637168 & 0.637168 & 0.637168 & 0.628319 & 0.637168 & 0.637168 \\
\hline $35 \%$ & 0.639640 & 0.603604 & 0.594595 & 0.630631 & 0.630631 & 0.630631 & 0.630631 & 0.621622 & 0.630631 & 0.630631 \\
\hline $40 \%$ & 0.632075 & 0.603774 & 0.594340 & 0.632075 & 0.632075 & 0.632075 & 0.632075 & 0.622642 & 0.632075 & 0.632075 \\
\hline $45 \%$ & 0.656863 & 0.617647 & 0.617647 & 0.647059 & 0.647059 & 0.647059 & 0.647059 & 0.637255 & 0.647059 & 0.647059 \\
\hline $50 \%$ & 0.647059 & 0.607843 & 0.598039 & 0.637255 & 0.637255 & 0.637255 & 0.637255 & 0.627451 & 0.637255 & 0.637255 \\
\hline $55 \%$ & 0.642857 & 0.612245 & 0.612245 & 0.642857 & 0.642857 & 0.642857 & 0.642857 & 0.632653 & 0.642857 & 0.642857 \\
\hline $60 \%$ & 0.663158 & 0.631579 & 0.631579 & 0.663158 & 0.663158 & 0.663158 & 0.663158 & 0.652632 & 0.663158 & 0.663158 \\
\hline $65 \%$ & 0.641304 & 0.630435 & 0.630435 & 0.663043 & 0.663043 & 0.663043 & 0.663043 & 0.652174 & 0.663043 & 0.663043 \\
\hline $70 \%$ & 0.655556 & 0.655556 & 0.655556 & 0.688889 & 0.688889 & 0.688889 & 0.688889 & 0.677778 & 0.688889 & 0.688889 \\
\hline $75 \%$ & 0.614458 & 0.614458 & 0.614458 & 0.638554 & 0.638554 & 0.638554 & 0.638554 & 0.638554 & 0.638554 & 0.638554 \\
\hline $80 \%$ & 0.653333 & 0.626667 & 0.626667 & 0.653333 & 0.653333 & 0.653333 & 0.653333 & 0.653333 & 0.653333 & 0.653333 \\
\hline $85 \%$ & 0.619718 & 0.605634 & 0.619718 & 0.633803 & 0.633803 & 0.633803 & 0.633803 & 0.633803 & 0.633803 & 0.633803 \\
\hline $90 \%$ & 0.600000 & 0.566667 & 0.583333 & 0.600000 & 0.600000 & 0.600000 & 0.600000 & 0.600000 & 0.600000 & 0.600000 \\
\hline $95 \%$ & 0.660000 & 0.620000 & 0.640000 & 0.660000 & 0.660000 & 0.660000 & 0.660000 & 0.660000 & 0.660000 & 0.660000 \\
\hline \multirow[t]{2}{*}{$100 \%$} & 0.659574 & 0.638298 & 0.680851 & 0.680851 & 0.680851 & 0.680851 & 0.680851 & 0.680851 & 0.680851 & 0.680851 \\
\hline & & & $\begin{array}{l}\text { Table } \\
\text { Randon }\end{array}$ & & 3. $\mathrm{I}$ & & Rates $\mathrm{f}$ & & & \\
\hline
\end{tabular}

Table 10 shows the same level of modifications as Table 8, but for the Inter-Trial Randomization, which means that the initial training and testing partition is randomly generated and reused for the set all modification amounts. Unlike Table 8, Table 10 shows a less sporadic set of values and when graphed looks like the upper graph of Figure 15. In Figure 15, only a few lines are plotted. This is a result of overlap as Table 10 shows. In Table 10, the Rank-1 Recognition Rates overlap for resolutions of 80x80, 100x100, 120x120, 140x140, 180x180, and 200x200. These overlapping Rank-1 Recognition Rates are also the second highest rates for all of the recognition rates for all resolutions. The highest Rank-1 Recognition Rates exist for a resolution of 20x20, however Xu et al. [36] warned against the use of this low of a dimensionality since the image is only machine- 
recognizable and harder for human-recognition. The same conclusions can be drawn from Table 11 as well (for nose length modifications).

\begin{tabular}{|r|c|c|c|c|c|c|c|c|c|c|}
\hline & $\mathbf{2 0 \times 2 0}$ & $\mathbf{4 0 \times 4 0}$ & $\mathbf{6 0 \times 6 0}$ & $\mathbf{8 0 \times 8 0}$ & $\mathbf{1 0 0 \times 1 0 0}$ & $\mathbf{1 2 0 \times 1 2 0}$ & $\mathbf{1 4 0 \times 1 4 0}$ & $\mathbf{1 6 0 \times 1 6 0}$ & $\mathbf{1 8 0 \times 1 8 0}$ & $\mathbf{2 0 0 \times 2 0 0}$ \\
\hline $\mathbf{- 2 0} \%$ & 0.661290 & 0.620968 & 0.620968 & 0.645161 & 0.645161 & 0.645161 & 0.645161 & 0.637097 & 0.645161 & 0.645161 \\
\hline $\mathbf{- 1 5} \%$ & 0.642857 & 0.603175 & 0.603175 & 0.626984 & 0.626984 & 0.626984 & 0.626984 & 0.619048 & 0.626984 & 0.626984 \\
\hline $\mathbf{- 1 0} \%$ & 0.633588 & 0.595420 & 0.595420 & 0.618321 & 0.618321 & 0.618321 & 0.618321 & 0.610687 & 0.618321 & 0.618321 \\
\hline $\mathbf{- 5} \%$ & 0.631579 & 0.593985 & 0.593985 & 0.616541 & 0.616541 & 0.616541 & 0.616541 & 0.609023 & 0.616541 & 0.616541 \\
\hline $\mathbf{5} \%$ & 0.629921 & 0.598425 & 0.598425 & 0.622047 & 0.622047 & 0.622047 & 0.622047 & 0.614173 & 0.622047 & 0.622047 \\
\hline $\mathbf{1 0} \%$ & 0.634921 & 0.603175 & 0.603175 & 0.626984 & 0.626984 & 0.626984 & 0.626984 & 0.619048 & 0.626984 & 0.626984 \\
\hline $\mathbf{1 5} \%$ & 0.629032 & 0.596774 & 0.596774 & 0.620968 & 0.620968 & 0.620968 & 0.620968 & 0.612903 & 0.620968 & 0.620968 \\
\hline $\mathbf{2 0} \%$ & 0.647541 & 0.614754 & 0.614754 & 0.639344 & 0.639344 & 0.639344 & 0.639344 & 0.631148 & 0.639344 & 0.639344 \\
\hline
\end{tabular}

Table 11: Resolution vs. Rank-1 Recognition Rates for Inter-Trial Randomization on Nose Length Modifications for LDA 


\section{APPENDIX B : CUMULATIVE MATCH CURVE}

Section 4.3 discusses the Cumulative Match Curves (CMC), which looks at the Recognition Rates for each trial as the number of ranks is increased. As the Rank ( $R N)$ increases, the top $N$ guessed identity labels are checked. If the correct identity label exists within the top $N$ labels, then its Cumulative Match Curve increases for that rank.

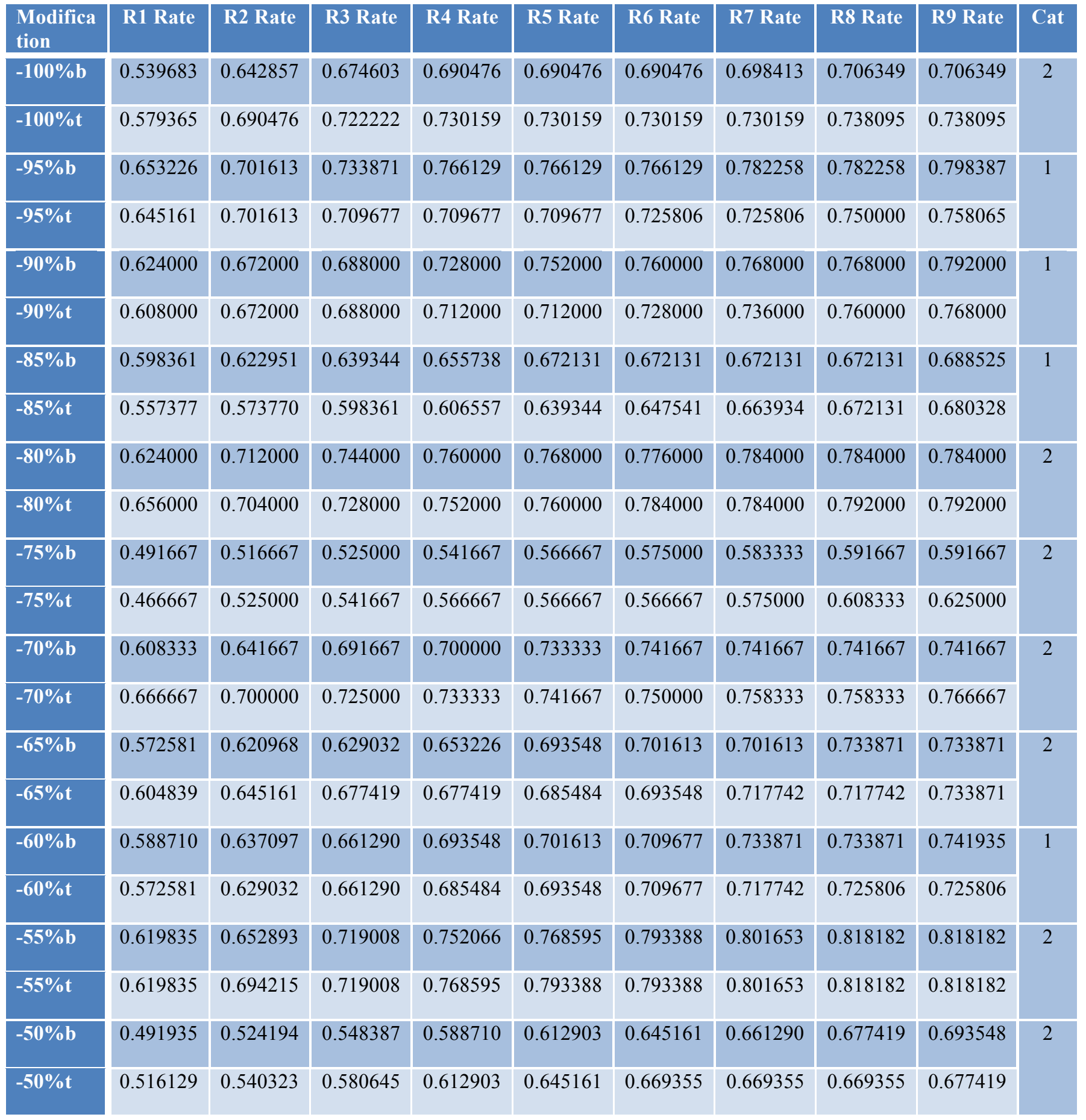




\begin{tabular}{|c|c|c|c|c|c|c|c|c|c|c|}
\hline$-45 \% b$ & 0.523077 & 0.538462 & 0.584615 & 0.584615 & 0.615385 & 0.630769 & 0.638462 & 0.638462 & 0.646154 & \multirow[t]{2}{*}{1} \\
\hline$-45 \% \mathrm{t}$ & 0.515385 & 0.561538 & 0.561538 & 0.576923 & 0.584615 & 0.630769 & 0.630769 & 0.630769 & 0.630769 & \\
\hline$-40 \% b$ & 0.551181 & 0.574803 & 0.582677 & 0.606299 & 0.614173 & 0.622047 & 0.637795 & 0.637795 & 0.645669 & \multirow[t]{2}{*}{1} \\
\hline$-40 \% t$ & 0.543307 & 0.566929 & 0.606299 & 0.614173 & 0.614173 & 0.622047 & 0.629921 & 0.629921 & 0.637795 & \\
\hline$-35 \% b$ & 0.587302 & 0.642857 & 0.666667 & 0.682540 & 0.690476 & 0.706349 & 0.706349 & 0.706349 & 0.714286 & \multirow[t]{2}{*}{1} \\
\hline$-35 \% t$ & 0.571429 & 0.595238 & 0.626984 & 0.642857 & 0.650794 & 0.666667 & 0.674603 & 0.674603 & 0.682540 & \\
\hline$-30 \% b$ & 0.562500 & 0.617188 & 0.648438 & 0.687500 & 0.695312 & 0.710938 & 0.726562 & 0.726562 & 0.742188 & \multirow[t]{2}{*}{2} \\
\hline$-30 \% t$ & 0.539062 & 0.625000 & 0.679688 & 0.710938 & 0.742188 & 0.773438 & 0.781250 & 0.781250 & 0.781250 & \\
\hline$-25 \% b$ & 0.587302 & 0.626984 & 0.658730 & 0.674603 & 0.674603 & 0.674603 & 0.682540 & 0.698413 & 0.698413 & \multirow[t]{2}{*}{2} \\
\hline$-25 \% t$ & 0.619048 & 0.642857 & 0.666667 & 0.690476 & 0.698413 & 0.722222 & 0.722222 & 0.722222 & 0.722222 & \\
\hline$-20 \% b$ & 0.587786 & 0.625954 & 0.656489 & 0.679389 & 0.694656 & 0.709924 & 0.717557 & 0.725191 & 0.732824 & \multirow[t]{2}{*}{2} \\
\hline$-20 \% t$ & 0.618321 & 0.656489 & 0.679389 & 0.694656 & 0.702290 & 0.732824 & 0.732824 & 0.732824 & 0.732824 & \\
\hline$-15 \% b$ & 0.608000 & 0.648000 & 0.672000 & 0.688000 & 0.720000 & 0.720000 & 0.728000 & 0.728000 & 0.728000 & \multirow[t]{2}{*}{2} \\
\hline$-15 \% t$ & 0.624000 & 0.664000 & 0.688000 & 0.712000 & 0.736000 & 0.736000 & 0.736000 & 0.736000 & 0.744000 & \\
\hline$-10 \% b$ & 0.562500 & 0.617188 & 0.648438 & 0.679688 & 0.695312 & 0.695312 & 0.695312 & 0.718750 & 0.718750 & \multirow[t]{2}{*}{1} \\
\hline$-10 \% t$ & 0.578125 & 0.609375 & 0.640625 & 0.671875 & 0.687500 & 0.687500 & 0.710938 & 0.726562 & 0.726562 & \\
\hline$-5 \% b$ & 0.692913 & 0.748031 & 0.755906 & 0.763780 & 0.771654 & 0.779528 & 0.779528 & 0.779528 & 0.787402 & \multirow[t]{2}{*}{2} \\
\hline$-5 \% t$ & 0.653543 & 0.724409 & 0.748031 & 0.755906 & 0.771654 & 0.795276 & 0.795276 & 0.795276 & 0.811024 & \\
\hline $5 \% b$ & 0.528000 & 0.584000 & 0.616000 & 0.648000 & 0.688000 & 0.688000 & 0.704000 & 0.704000 & 0.720000 & \multirow[t]{2}{*}{1} \\
\hline $5 \% t$ & 0.552000 & 0.576000 & 0.608000 & 0.632000 & 0.648000 & 0.672000 & 0.680000 & 0.680000 & 0.712000 & \\
\hline $10 \% b$ & 0.519380 & 0.519380 & 0.534884 & 0.565891 & 0.573643 & 0.589147 & 0.596899 & 0.604651 & 0.604651 & \multirow[t]{2}{*}{1} \\
\hline $10 \% \mathrm{t}$ & 0.488372 & 0.511628 & 0.534884 & 0.534884 & 0.550388 & 0.550388 & 0.565891 & 0.565891 & 0.573643 & \\
\hline $15 \% b$ & 0.533898 & 0.601695 & 0.627119 & 0.661017 & 0.686441 & 0.720339 & 0.720339 & 0.737288 & 0.745763 & \multirow[t]{2}{*}{2} \\
\hline $15 \% t$ & 0.550847 & 0.635593 & 0.661017 & 0.711864 & 0.711864 & 0.728814 & 0.745763 & 0.745763 & 0.754237 & \\
\hline $20 \% b$ & 0.504202 & 0.554622 & 0.571429 & 0.596639 & 0.613445 & 0.630252 & 0.638655 & 0.655462 & 0.655462 & \multirow[t]{2}{*}{1} \\
\hline $20 \% t$ & 0.512605 & 0.554622 & 0.554622 & 0.571429 & 0.579832 & 0.588235 & 0.605042 & 0.613445 & 0.621849 & \\
\hline
\end{tabular}




\begin{tabular}{|c|c|c|c|c|c|c|c|c|c|c|}
\hline $25 \% b$ & 0.564103 & 0.581197 & 0.589744 & 0.606838 & 0.606838 & 0.615385 & 0.632479 & 0.632479 & 0.632479 & \multirow[t]{2}{*}{2} \\
\hline $25 \% t$ & 0.521368 & 0.5555566 & 0.564103 & 0.598291 & 0.606838 & 0.623932 & 0.632479 & 0.641026 & 0.649573 & \\
\hline $30 \% b$ & 0.559633 & 0.651376 & 0.688073 & 0.724771 & 0.733945 & 0.743119 & 0.770642 & 0.770642 & 0.788991 & \multirow[t]{2}{*}{1} \\
\hline $30 \% t$ & 0.577982 & 0.633028 & 0.688073 & 0.697248 & 0.715596 & 0.733945 & 0.733945 & 0.743119 & 0.761468 & \\
\hline $35 \% b$ & 0.478992 & 0.571429 & 0.579832 & 0.588235 & 0.596639 & 0.596639 & 0.613445 & 0.630252 & 0.630252 & \multirow[t]{2}{*}{1} \\
\hline $35 \% t$ & 0.445378 & 0.495798 & 0.546218 & 0.554622 & 0.554622 & 0.554622 & 0.563025 & 0.571429 & 0.579832 & \\
\hline $40 \% b$ & 0.609524 & 0.657143 & 0.666667 & 0.666667 & 0.695238 & 0.714286 & 0.733333 & 0.742857 & 0.742857 & \multirow[t]{2}{*}{1} \\
\hline $40 \% t$ & 0.561905 & 0.628571 & 0.657143 & 0.666667 & 0.676190 & 0.695238 & 0.733333 & 0.733333 & 0.742857 & \\
\hline $45 \% b$ & 0.529412 & 0.568627 & 0.588235 & 0.588235 & 0.607843 & 0.617647 & 0.627451 & 0.666667 & 0.666667 & \multirow[t]{2}{*}{2} \\
\hline $45 \% t$ & 0.509804 & 0.568627 & 0.588235 & 0.598039 & 0.617647 & 0.637255 & 0.647059 & 0.656863 & 0.666667 & \\
\hline $50 \% b$ & 0.613208 & 0.679245 & 0.698113 & 0.716981 & 0.735849 & 0.735849 & 0.735849 & 0.745283 & 0.754717 & \multirow[t]{2}{*}{1} \\
\hline $50 \% t$ & 0.575472 & 0.622642 & 0.632075 & 0.632075 & 0.660377 & 0.669811 & 0.669811 & 0.679245 & 0.688679 & \\
\hline $55 \% b$ & 0.551020 & 0.591837 & 0.602041 & 0.622449 & 0.622449 & 0.642857 & 0.642857 & 0.663265 & 0.663265 & \multirow[t]{2}{*}{1} \\
\hline $55 \% t$ & 0.479592 & 0.510204 & 0.540816 & 0.581633 & 0.591837 & 0.591837 & 0.602041 & 0.602041 & 0.602041 & \\
\hline $60 \% b$ & 0.630000 & 0.670000 & 0.700000 & 0.740000 & 0.740000 & 0.760000 & 0.760000 & 0.760000 & 0.770000 & \multirow[t]{2}{*}{1} \\
\hline $60 \% \mathrm{t}$ & 0.510000 & 0.580000 & 0.580000 & 0.610000 & 0.640000 & 0.660000 & 0.690000 & 0.690000 & 0.690000 & \\
\hline $65 \% b$ & 0.483516 & 0.505495 & 0.538462 & 0.549451 & 0.571429 & 0.604396 & 0.626374 & 0.648352 & 0.670330 & \multirow[t]{2}{*}{1} \\
\hline $65 \% t$ & 0.329670 & 0.417582 & 0.461538 & 0.505495 & 0.549451 & 0.571429 & 0.604396 & 0.604396 & 0.626374 & \\
\hline $70 \% b$ & 0.528090 & 0.595506 & 0.640449 & 0.685393 & 0.707865 & 0.719101 & 0.730337 & 0.730337 & 0.741573 & \multirow[t]{2}{*}{1} \\
\hline $70 \% t$ & 0.438202 & 0.460674 & 0.483146 & 0.516854 & 0.528090 & 0.550562 & 0.561798 & 0.573034 & 0.584270 & \\
\hline $75 \% b$ & 0.653846 & 0.666667 & 0.692308 & 0.717949 & 0.717949 & 0.730769 & 0.730769 & 0.730769 & 0.730769 & \multirow[t]{2}{*}{1} \\
\hline $75 \% t$ & 0.474359 & 0.551282 & 0.615385 & 0.641026 & 0.653846 & 0.666667 & 0.666667 & 0.679487 & 0.717949 & \\
\hline $80 \% b$ & 0.631579 & 0.657895 & 0.671053 & 0.697368 & 0.710526 & 0.710526 & 0.710526 & 0.710526 & 0.723684 & \multirow[t]{2}{*}{1} \\
\hline $80 \% t$ & 0.394737 & 0.460526 & 0.500000 & 0.500000 & 0.552632 & 0.552632 & 0.552632 & 0.552632 & 0.565789 & \\
\hline $85 \% b$ & 0.571429 & 0.614286 & 0.671429 & 0.714286 & 0.728571 & 0.728571 & 0.757143 & 0.757143 & 0.785714 & \multirow[t]{2}{*}{1} \\
\hline $85 \% \mathrm{t}$ & 0.414286 & 0.457143 & 0.514286 & 0.528571 & 0.600000 & 0.628571 & 0.642857 & 0.685714 & 0.700000 & \\
\hline
\end{tabular}




\begin{tabular}{|l|l|l|l|l|l|l|l|l|l|l|}
\hline $\mathbf{9 0 \% b}$ & 0.612903 & 0.661290 & 0.693548 & 0.709677 & 0.725806 & 0.725806 & 0.725806 & 0.741935 & 0.758065 & 1 \\
\hline $\mathbf{9 0 \% t}$ & 0.354839 & 0.403226 & 0.419355 & 0.451613 & 0.483871 & 0.516129 & 0.532258 & 0.564516 & 0.580645 \\
\hline $\mathbf{9 5 \% b}$ & 0.532258 & 0.596774 & 0.596774 & 0.629032 & 0.645161 & 0.661290 & 0.677419 & 0.709677 & 0.709677 & 1 \\
\hline $\mathbf{9 5 \% t}$ & 0.387097 & 0.467742 & 0.516129 & 0.564516 & 0.580645 & 0.612903 & 0.612903 & 0.629032 & 0.661290 \\
\hline $\mathbf{1 0 0 \% b}$ & 0.673913 & 0.717391 & 0.739130 & 0.739130 & 0.739130 & 0.739130 & 0.739130 & 0.739130 & 0.739130 & 1 \\
\hline $\mathbf{1 0 0 \% t}$ & 0.456522 & 0.521739 & 0.543478 & 0.543478 & 0.608696 & 0.608696 & 0.630435 & 0.630435 & 0.652174 \\
\hline
\end{tabular}

Table 12: Cumulative Match Curve For Intra-Trial Randomization on Nose Width Modifications for LDA

Table 12 shows multiple lines for the Intra-Trial Randomization for the Nose Width Modifications. When looking at the Modifications, two rows for each modification are shown, one for the baseline (b) for the unmodified test images, and the treatment ( $\mathrm{t}$ ) row for the modified test images. For each row, the rank $N(1-9)$ recognition rate is shown as R1 Rate (meaning Rank-1 Recognition Rate), and the Category Number (as explained in Section 3.4.2). Each pair modification results have a single category number, since the two are compared between the other. Table 12 is preferred over the graphs in Section 4.4.1 since this provides a clear numerical comparison between the baseline and treatment curves. The alternative would have been to provide 40 Nose Width and 8 Nose Length Graphs for Intra-Trial Modifications, then an additional 48 for Inter-Trial Modifications. The table allows for clarity as well as the ability to reconstruct the graphs if deemed necessary. As Table 12 shows, for 30\% Nose Width Modifications to $100 \%$, they are all category 1, which is that the baseline curve was higher than the treatment curve. This result and more conclusions can be found in Section 4.4.1.

\begin{tabular}{|r|l|l|l|l|l|l|l|l|l|l|}
\hline $\begin{array}{c}\text { Modific } \\
\text { ation }\end{array}$ & R1 Rate & R2 Rate & R3 Rate & R4 Rate & R5 Rate & R6 Rate & R7 Rate & R8 Rate & R9 Rate & Cat \\
\hline $\mathbf{- 2 0 \% b}$ & 0.500000 & 0.540984 & 0.557377 & 0.565574 & 0.573770 & 0.581967 & 0.590164 & 0.606557 & 0.606557 \\
\hline $\mathbf{- 2 0 \%}$ & 0.459016 & 0.500000 & 0.508197 & 0.516393 & 0.532787 & 0.557377 & 0.565574 & 0.573770 & 0.573770 \\
\hline $\mathbf{- 1 5} \% \mathbf{b}$ & 0.610687 & 0.656489 & 0.725191 & 0.763359 & 0.786260 & 0.801527 & 0.801527 & 0.801527 & 0.809160 & 1 \\
\hline $\mathbf{- 1 5} \% \mathbf{t}$ & 0.641221 & 0.709924 & 0.732824 & 0.732824 & 0.748092 & 0.755725 & 0.763359 & 0.770992 & 0.786260 \\
\hline $\mathbf{- 1 0} \% \mathbf{b}$ & 0.484615 & 0.569231 & 0.592308 & 0.615385 & 0.623077 & 0.630769 & 0.646154 & 0.661538 & 0.669231 & 2 \\
\hline $\mathbf{- 1 0} \% \mathbf{t}$ & 0.492308 & 0.546154 & 0.584615 & 0.615385 & 0.630769 & 0.638462 & 0.653846 & 0.661538 & 0.661538 \\
\hline
\end{tabular}




\begin{tabular}{|r|l|l|l|l|l|l|l|l|l|l|}
\hline $\mathbf{5 \% b}$ & 0.507463 & 0.552239 & 0.567164 & 0.582090 & 0.604478 & 0.619403 & 0.619403 & 0.626866 & 0.641791 & 1 \\
\hline $\mathbf{- 5 \% t}$ & 0.514925 & 0.544776 & 0.574627 & 0.582090 & 0.597015 & 0.604478 & 0.604478 & 0.619403 & 0.634328 \\
\hline $\mathbf{5 \% b}$ & 0.632000 & 0.664000 & 0.672000 & 0.704000 & 0.704000 & 0.728000 & 0.736000 & 0.744000 & 0.744000 & 1 \\
\hline $\mathbf{5 \% t}$ & 0.616000 & 0.648000 & 0.688000 & 0.696000 & 0.704000 & 0.712000 & 0.720000 & 0.736000 & 0.744000 \\
\hline $\mathbf{1 0} \% \mathbf{b}$ & 0.542636 & 0.558140 & 0.565891 & 0.589147 & 0.589147 & 0.596899 & 0.596899 & 0.604651 & 0.604651 & 2 \\
\hline $\mathbf{1 0 \% t}$ & 0.542636 & 0.612403 & 0.620155 & 0.627907 & 0.635659 & 0.651163 & 0.651163 & 0.651163 & 0.651163 & \\
\hline $\mathbf{1 5 \% b}$ & 0.524590 & 0.540984 & 0.573770 & 0.598361 & 0.622951 & 0.631148 & 0.639344 & 0.680328 & 0.680328 & 2 \\
\hline $\mathbf{1 5 \% t}$ & 0.540984 & 0.565574 & 0.598361 & 0.631148 & 0.639344 & 0.680328 & 0.688525 & 0.696721 & 0.713115 \\
\hline $\mathbf{2 0} \% \mathbf{b}$ & 0.603306 & 0.652893 & 0.669421 & 0.685950 & 0.685950 & 0.694215 & 0.710744 & 0.719008 & 0.719008 & 2 \\
\hline $\mathbf{2 0 \% t}$ & 0.578512 & 0.644628 & 0.677686 & 0.710744 & 0.727273 & 0.727273 & 0.735537 & 0.743802 & 0.752066 \\
\hline
\end{tabular}

Table 13: Cumulative Match Curve For Intra-Trial Randomization on Nose Length Modifications for LDA

Similar to Table 12, Table 13 shows all of the Intra-Trial Nose Length Modifications. In Table 13, most of the Category 2 (where the baseline curve rates are less than the treatment) is close together and finish slightly higher than the other. Even for the Category 1 rates, 0.03 difference between the treatment and the baseline curves exists. The rest of the results and comparisons can be seen in Section 4.4.1.

\begin{tabular}{|c|c|c|c|c|c|c|c|c|c|c|}
\hline $\begin{array}{c}\text { Modific } \\
\text { ation }\end{array}$ & R1 Rate & R2 Rate & R3 Rate & R4 Rate & R5 Rate & R6 Rate & R7 Rate & R8 Rate & R9 Rate & Cat \\
\hline $\mathbf{- 1 0 0 \% b}$ & 0.632812 & 0.695312 & 0.703125 & 0.718750 & 0.718750 & 0.726562 & 0.726562 & 0.734375 & 0.742188 & 1 \\
\hline $\mathbf{- 1 0 0 \% t}$ & 0.648438 & 0.671875 & 0.687500 & 0.703125 & 0.710938 & 0.718750 & 0.734375 & 0.734375 & 0.734375 \\
\hline $\mathbf{- 9 5 \% b}$ & 0.632812 & 0.695312 & 0.703125 & 0.718750 & 0.718750 & 0.726562 & 0.726562 & 0.734375 & 0.742188 & 1 \\
\hline $\mathbf{- 9 5} \% \mathbf{t}$ & 0.648438 & 0.695312 & 0.703125 & 0.703125 & 0.710938 & 0.710938 & 0.726562 & 0.726562 & 0.734375 \\
\hline $\mathbf{- 9 0 \% b}$ & 0.632000 & 0.696000 & 0.704000 & 0.720000 & 0.720000 & 0.728000 & 0.728000 & 0.736000 & 0.744000 & 2 \\
\hline $\mathbf{- 9 0 \% t}$ & 0.656000 & 0.696000 & 0.696000 & 0.704000 & 0.728000 & 0.728000 & 0.728000 & 0.728000 & 0.744000 \\
\hline $\mathbf{- 8 5} \% \mathbf{b}$ & 0.632000 & 0.696000 & 0.704000 & 0.720000 & 0.720000 & 0.728000 & 0.728000 & 0.736000 & 0.736000 & 1 \\
\hline $\mathbf{- 8 5} \% \mathbf{t}$ & 0.624000 & 0.688000 & 0.696000 & 0.696000 & 0.712000 & 0.712000 & 0.712000 & 0.712000 & 0.712000 \\
\hline
\end{tabular}




\begin{tabular}{|c|c|c|c|c|c|c|c|c|c|c|}
\hline$-80 \% b$ & 0.629032 & 0.693548 & 0.701613 & 0.717742 & 0.717742 & 0.725806 & 0.725806 & 0.733871 & 0.733871 & \multirow[t]{2}{*}{1} \\
\hline$-80 \% t$ & 0.620968 & 0.685484 & 0.701613 & 0.701613 & 0.717742 & 0.717742 & 0.717742 & 0.717742 & 0.717742 & \\
\hline$-75 \% b$ & 0.632000 & 0.696000 & 0.704000 & 0.720000 & 0.720000 & 0.728000 & 0.728000 & 0.736000 & 0.736000 & \multirow[t]{2}{*}{1} \\
\hline$-75 \% \mathrm{t}$ & 0.640000 & 0.664000 & 0.688000 & 0.696000 & 0.704000 & 0.712000 & 0.712000 & 0.712000 & 0.720000 & \\
\hline$-70 \% b$ & 0.634921 & 0.698413 & 0.706349 & 0.722222 & 0.722222 & 0.730159 & 0.730159 & 0.738095 & 0.746032 & \multirow[t]{2}{*}{1} \\
\hline$-70 \% t$ & 0.650794 & 0.698413 & 0.706349 & 0.706349 & 0.722222 & 0.722222 & 0.722222 & 0.722222 & 0.730159 & \\
\hline$-65 \% b$ & 0.640000 & 0.704000 & 0.712000 & 0.720000 & 0.720000 & 0.728000 & 0.728000 & 0.736000 & 0.744000 & \multirow[t]{2}{*}{1} \\
\hline$-65 \% t$ & 0.624000 & 0.672000 & 0.696000 & 0.704000 & 0.704000 & 0.712000 & 0.712000 & 0.720000 & 0.720000 & \\
\hline$-60 \% b$ & 0.642857 & 0.706349 & 0.714286 & 0.722222 & 0.722222 & 0.730159 & 0.730159 & 0.738095 & 0.746032 & \multirow[t]{2}{*}{1} \\
\hline$-60 \% t$ & 0.619048 & 0.674603 & 0.682540 & 0.682540 & 0.698413 & 0.714286 & 0.722222 & 0.722222 & 0.722222 & \\
\hline$-55 \% b$ & 0.645161 & 0.709677 & 0.717742 & 0.725806 & 0.725806 & 0.733871 & 0.733871 & 0.741935 & 0.741935 & \multirow[t]{2}{*}{1} \\
\hline$-55 \% t$ & 0.637097 & 0.669355 & 0.685484 & 0.685484 & 0.709677 & 0.733871 & 0.733871 & 0.733871 & 0.733871 & \\
\hline$-50 \% b$ & 0.632000 & 0.696000 & 0.704000 & 0.720000 & 0.720000 & 0.728000 & 0.728000 & 0.736000 & 0.736000 & \multirow[t]{2}{*}{1} \\
\hline$-50 \% t$ & 0.608000 & 0.680000 & 0.688000 & 0.688000 & 0.696000 & 0.704000 & 0.704000 & 0.704000 & 0.712000 & \\
\hline$-45 \% b$ & 0.640000 & 0.704000 & 0.712000 & 0.720000 & 0.720000 & 0.728000 & 0.728000 & 0.736000 & 0.736000 & \multirow[t]{2}{*}{2} \\
\hline$-45 \% t$ & 0.632000 & 0.688000 & 0.712000 & 0.720000 & 0.720000 & 0.720000 & 0.728000 & 0.728000 & 0.736000 & \\
\hline$-40 \% b$ & 0.634921 & 0.698413 & 0.706349 & 0.714286 & 0.714286 & 0.722222 & 0.722222 & 0.730159 & 0.730159 & \multirow[t]{2}{*}{1} \\
\hline$-40 \% \mathrm{t}$ & 0.626984 & 0.682540 & 0.682540 & 0.698413 & 0.706349 & 0.706349 & 0.706349 & 0.706349 & 0.706349 & \\
\hline$-35 \% b$ & 0.637795 & 0.708661 & 0.716535 & 0.724409 & 0.724409 & 0.732283 & 0.732283 & 0.740157 & 0.740157 & \multirow[t]{2}{*}{2} \\
\hline$-35 \% t$ & 0.645669 & 0.716535 & 0.716535 & 0.732283 & 0.732283 & 0.732283 & 0.732283 & 0.732283 & 0.732283 & \\
\hline$-30 \% b$ & 0.637795 & 0.708661 & 0.716535 & 0.724409 & 0.724409 & 0.732283 & 0.732283 & 0.740157 & 0.740157 & \multirow[t]{2}{*}{1} \\
\hline$-30 \% t$ & 0.614173 & 0.700787 & 0.708661 & 0.716535 & 0.716535 & 0.724409 & 0.724409 & 0.732283 & 0.732283 & \\
\hline$-25 \% b$ & 0.625000 & 0.695312 & 0.703125 & 0.710938 & 0.710938 & 0.718750 & 0.718750 & 0.726562 & 0.726562 & \multirow[t]{2}{*}{2} \\
\hline$-25 \% t$ & 0.625000 & 0.687500 & 0.703125 & 0.710938 & 0.710938 & 0.726562 & 0.726562 & 0.726562 & 0.726562 & \\
\hline$-20 \% b$ & 0.620155 & 0.689922 & 0.697674 & 0.705426 & 0.705426 & 0.713178 & 0.713178 & 0.720930 & 0.728682 & \multirow[t]{2}{*}{2} \\
\hline$-20 \% t$ & 0.643411 & 0.689922 & 0.705426 & 0.713178 & 0.728682 & 0.728682 & 0.728682 & 0.736434 & 0.744186 & \\
\hline
\end{tabular}




\begin{tabular}{|c|c|c|c|c|c|c|c|c|c|c|}
\hline$-15 \% b$ & 0.625000 & 0.695312 & 0.703125 & 0.710938 & 0.710938 & 0.718750 & 0.718750 & 0.726562 & 0.734375 & \multirow[t]{2}{*}{1} \\
\hline$-15 \% t$ & 0.609375 & 0.664062 & 0.671875 & 0.695312 & 0.703125 & 0.710938 & 0.710938 & 0.726562 & 0.734375 & \\
\hline$-10 \% b$ & 0.620155 & 0.689922 & 0.697674 & 0.705426 & 0.705426 & 0.713178 & 0.713178 & 0.720930 & 0.728682 & \multirow[t]{2}{*}{2} \\
\hline$-10 \% t$ & 0.620155 & 0.666667 & 0.682171 & 0.689922 & 0.689922 & 0.713178 & 0.713178 & 0.720930 & 0.728682 & \\
\hline$-5 \% b$ & 0.622047 & 0.692913 & 0.700787 & 0.708661 & 0.708661 & 0.716535 & 0.716535 & 0.724409 & 0.732283 & \multirow[t]{2}{*}{2} \\
\hline$-5 \% t$ & 0.629921 & 0.669291 & 0.685039 & 0.692913 & 0.708661 & 0.724409 & 0.724409 & 0.724409 & 0.732283 & \\
\hline $5 \% b$ & 0.635659 & 0.705426 & 0.713178 & 0.728682 & 0.728682 & 0.736434 & 0.736434 & 0.744186 & 0.744186 & \multirow[t]{2}{*}{1} \\
\hline $5 \% t$ & 0.589147 & 0.658915 & 0.682171 & 0.705426 & 0.713178 & 0.720930 & 0.720930 & 0.720930 & 0.720930 & \\
\hline $10 \% \mathrm{~b}$ & 0.640625 & 0.710938 & 0.718750 & 0.734375 & 0.734375 & 0.742188 & 0.742188 & 0.750000 & 0.750000 & \multirow[t]{2}{*}{1} \\
\hline $10 \% \mathrm{t}$ & 0.601562 & 0.679688 & 0.703125 & 0.718750 & 0.726562 & 0.726562 & 0.726562 & 0.726562 & 0.726562 & \\
\hline $15 \% b$ & 0.639344 & 0.704918 & 0.704918 & 0.721311 & 0.721311 & 0.729508 & 0.729508 & 0.737705 & 0.737705 & \multirow[t]{2}{*}{1} \\
\hline $15 \% \mathrm{t}$ & 0.598361 & 0.663934 & 0.672131 & 0.680328 & 0.696721 & 0.696721 & 0.704918 & 0.704918 & 0.704918 & \\
\hline $20 \% b$ & 0.623932 & 0.700855 & 0.700855 & 0.717949 & 0.717949 & 0.726496 & 0.726496 & 0.735043 & 0.735043 & \multirow[t]{2}{*}{1} \\
\hline $20 \% \mathrm{t}$ & 0.598291 & 0.641026 & 0.658120 & 0.675214 & 0.675214 & 0.683761 & 0.692308 & 0.692308 & 0.692308 & \\
\hline $25 \% b$ & 0.631579 & 0.710526 & 0.710526 & 0.728070 & 0.728070 & 0.736842 & 0.736842 & 0.745614 & 0.745614 & \multirow[t]{2}{*}{1} \\
\hline $25 \% \mathrm{t}$ & 0.570175 & 0.649123 & 0.675439 & 0.675439 & 0.675439 & 0.675439 & 0.675439 & 0.684211 & 0.692982 & \\
\hline $30 \% b$ & 0.637168 & 0.699115 & 0.699115 & 0.707965 & 0.707965 & 0.716814 & 0.716814 & 0.716814 & 0.725664 & \multirow[t]{2}{*}{1} \\
\hline $30 \% t$ & 0.575221 & 0.619469 & 0.646018 & 0.654867 & 0.654867 & 0.663717 & 0.663717 & 0.672566 & 0.672566 & \\
\hline $35 \% b$ & 0.630631 & 0.702703 & 0.711712 & 0.720721 & 0.720721 & 0.729730 & 0.729730 & 0.729730 & 0.729730 & \multirow[t]{2}{*}{1} \\
\hline $35 \% \mathrm{t}$ & 0.549550 & 0.639640 & 0.648649 & 0.657658 & 0.675676 & 0.675676 & 0.675676 & 0.684685 & 0.684685 & \\
\hline $40 \% b$ & 0.632075 & 0.707547 & 0.707547 & 0.716981 & 0.716981 & 0.716981 & 0.716981 & 0.726415 & 0.735849 & \multirow[t]{2}{*}{1} \\
\hline $40 \% \mathrm{t}$ & 0.594340 & 0.641509 & 0.679245 & 0.679245 & 0.679245 & 0.679245 & 0.698113 & 0.698113 & 0.698113 & \\
\hline $45 \% b$ & 0.647059 & 0.715686 & 0.725490 & 0.735294 & 0.735294 & 0.735294 & 0.735294 & 0.745098 & 0.754902 & \multirow[t]{2}{*}{1} \\
\hline $45 \% t$ & 0.519608 & 0.598039 & 0.647059 & 0.666667 & 0.676471 & 0.686275 & 0.696078 & 0.705882 & 0.705882 & \\
\hline $50 \% b$ & 0.637255 & 0.715686 & 0.715686 & 0.725490 & 0.725490 & 0.725490 & 0.725490 & 0.735294 & 0.745098 & \multirow[t]{2}{*}{1} \\
\hline $50 \% t$ & 0.588235 & 0.676471 & 0.686275 & 0.696078 & 0.705882 & 0.705882 & 0.705882 & 0.705882 & 0.705882 & \\
\hline
\end{tabular}




\begin{tabular}{|c|c|c|c|c|c|c|c|c|c|c|}
\hline $55 \% b$ & 0.642857 & 0.714286 & 0.714286 & 0.724490 & 0.724490 & 0.734694 & 0.734694 & 0.744898 & 0.755102 & \multirow[t]{2}{*}{1} \\
\hline $55 \% \mathrm{t}$ & 0.551020 & 0.612245 & 0.653061 & 0.663265 & 0.673469 & 0.673469 & 0.673469 & 0.683673 & 0.693878 & \\
\hline $60 \% \mathrm{~b}$ & 0.663158 & 0.736842 & 0.736842 & 0.747368 & 0.747368 & 0.747368 & 0.747368 & 0.747368 & 0.757895 & \multirow[t]{2}{*}{1} \\
\hline $60 \% \mathrm{t}$ & 0.547368 & 0.621053 & 0.652632 & 0.663158 & 0.663158 & 0.673684 & 0.673684 & 0.673684 & 0.684211 & \\
\hline $65 \% b$ & 0.663043 & 0.728261 & 0.728261 & 0.739130 & 0.739130 & 0.739130 & 0.739130 & 0.739130 & 0.750000 & \multirow[t]{2}{*}{1} \\
\hline $65 \% t$ & 0.500000 & 0.597826 & 0.641304 & 0.673913 & 0.684783 & 0.684783 & 0.684783 & 0.684783 & 0.695652 & \\
\hline $70 \% b$ & 0.688889 & 0.733333 & 0.733333 & 0.744444 & 0.744444 & 0.744444 & 0.744444 & 0.744444 & 0.744444 & \multirow[t]{2}{*}{1} \\
\hline $70 \% t$ & 0.500000 & 0.566667 & 0.588889 & 0.600000 & 0.644444 & 0.655556 & 0.655556 & 0.655556 & 0.688889 & \\
\hline $75 \% b$ & 0.638554 & 0.698795 & 0.698795 & 0.710843 & 0.710843 & 0.710843 & 0.710843 & 0.710843 & 0.722892 & \multirow[t]{2}{*}{1} \\
\hline $75 \% t$ & 0.518072 & 0.614458 & 0.650602 & 0.662651 & 0.674699 & 0.674699 & 0.674699 & 0.686747 & 0.698795 & \\
\hline $80 \% b$ & 0.653333 & 0.733333 & 0.733333 & 0.746667 & 0.746667 & 0.746667 & 0.746667 & 0.746667 & 0.760000 & \multirow[t]{2}{*}{1} \\
\hline $80 \% t$ & 0.466667 & 0.546667 & 0.600000 & 0.613333 & 0.613333 & 0.613333 & 0.626667 & 0.640000 & 0.640000 & \\
\hline $85 \% b$ & 0.633803 & 0.704225 & 0.704225 & 0.718310 & 0.718310 & 0.718310 & 0.718310 & 0.718310 & 0.732394 & \multirow[t]{2}{*}{1} \\
\hline $85 \% t$ & 0.535211 & 0.577465 & 0.605634 & 0.633803 & 0.661972 & 0.676056 & 0.690141 & 0.690141 & 0.704225 & \\
\hline $90 \% b$ & 0.600000 & 0.650000 & 0.650000 & 0.683333 & 0.683333 & 0.683333 & 0.683333 & 0.683333 & 0.700000 & \multirow[t]{2}{*}{1} \\
\hline $90 \% \mathrm{t}$ & 0.450000 & 0.516667 & 0.550000 & 0.600000 & 0.600000 & 0.616667 & 0.616667 & 0.633333 & 0.650000 & \\
\hline $95 \% b$ & 0.660000 & 0.720000 & 0.720000 & 0.760000 & 0.760000 & 0.760000 & 0.760000 & 0.760000 & 0.760000 & \multirow[t]{2}{*}{1} \\
\hline $95 \% \mathrm{t}$ & 0.440000 & 0.520000 & 0.560000 & 0.580000 & 0.640000 & 0.640000 & 0.640000 & 0.640000 & 0.660000 & \\
\hline $100 \% \mathrm{~b}$ & 0.680851 & 0.702128 & 0.702128 & 0.723404 & 0.723404 & 0.723404 & 0.723404 & 0.723404 & 0.723404 & \multirow[t]{2}{*}{1} \\
\hline $100 \% t$ & 0.468085 & 0.510638 & 0.617021 & 0.638298 & 0.659574 & 0.659574 & 0.659574 & 0.659574 & 0.702128 & \\
\hline
\end{tabular}

Table 14: Cumulative Match Curve For Inter-Trial Randomization on Nose Width Modifications for LDA

Just like Table 12 shows the nose width modifications for the Intra-Trial Randomization, Table 14 shows the same nose width modifications but for the Inter-Trial Randomization. In Table 14, the same training images are used and the same test sets (unmodified and modified) are used. Similar to Table 12, as the nose is enlarged the treatment (modification) curve is less than that of the baseline rates. However where Table 12 has a consistent Category 1 from $+30 \%$ to $+100 \%$, Table 
14 is consistent for $+5 \%$ to $100 \%$ modification. Unlike Table 13 , Table 14 has a higher difference between the baseline and treatment curves especially for positive nose width modifications.

\begin{tabular}{|c|c|c|c|c|c|c|c|c|c|c|}
\hline $\begin{array}{l}\text { Modifi } \\
\text { cation }\end{array}$ & R1 Rate & R2 Rate & R3 Rate & R4 Rate & R5 Rate & R6 Rate & R7 Rate & R8 Rate & R9 Rate & Cat. \\
\hline$-20 \% b$ & 0.645161 & 0.709677 & 0.717742 & 0.733871 & 0.733871 & 0.741935 & 0.741935 & 0.750000 & 0.758065 & 1 \\
\hline$-20 \% t$ & 0.645161 & 0.685484 & 0.709677 & 0.709677 & 0.717742 & 0.741935 & 0.741935 & 0.741935 & 0.741935 & \\
\hline$-15 \% b$ & 0.626984 & 0.690476 & 0.698413 & 0.714286 & 0.714286 & 0.722222 & 0.722222 & 0.730159 & 0.730159 & 2 \\
\hline$-15 \% t$ & 0.619048 & 0.674603 & 0.706349 & 0.714286 & 0.714286 & 0.722222 & 0.722222 & 0.722222 & 0.722222 & \\
\hline$-10 \% b$ & 0.618321 & 0.687023 & 0.694656 & 0.709924 & 0.709924 & 0.717557 & 0.717557 & 0.725191 & 0.725191 & 1 \\
\hline$-10 \% t$ & 0.618321 & 0.671756 & 0.694656 & 0.702290 & 0.709924 & 0.709924 & 0.717557 & 0.717557 & 0.717557 & \\
\hline$-5 \% b$ & 0.616541 & 0.684211 & 0.691729 & 0.706767 & 0.706767 & 0.714286 & 0.714286 & 0.721805 & 0.721805 & 2 \\
\hline$-5 \% \mathrm{t}$ & 0.631579 & 0.684211 & 0.699248 & 0.714286 & 0.714286 & 0.721805 & 0.721805 & 0.729323 & 0.729323 & \\
\hline $5 \% b$ & 0.622047 & 0.692913 & 0.700787 & 0.708661 & 0.708661 & 0.716535 & 0.716535 & 0.724409 & 0.732283 & 2 \\
\hline $5 \% \mathrm{t}$ & 0.598425 & 0.677165 & 0.685039 & 0.692913 & 0.724409 & 0.724409 & 0.732283 & 0.732283 & 0.732283 & \\
\hline $10 \% b$ & 0.626984 & 0.698413 & 0.706349 & 0.714286 & 0.714286 & 0.722222 & 0.722222 & 0.730159 & 0.738095 & 1 \\
\hline $10 \% t$ & 0.603175 & 0.682540 & 0.690476 & 0.698413 & 0.714286 & 0.714286 & 0.722222 & 0.730159 & 0.730159 & \\
\hline $15 \% b$ & 0.620968 & 0.685484 & 0.693548 & 0.701613 & 0.701613 & 0.709677 & 0.709677 & 0.717742 & 0.725806 & 1 \\
\hline $15 \% \mathrm{t}$ & 0.588710 & 0.653226 & 0.669355 & 0.693548 & 0.701613 & 0.701613 & 0.709677 & 0.717742 & 0.717742 & \\
\hline $20 \% b$ & 0.639344 & 0.704918 & 0.713115 & 0.721311 & 0.721311 & 0.729508 & 0.729508 & 0.737705 & 0.745902 & 1 \\
\hline $20 \% t$ & 0.581967 & 0.606557 & 0.647541 & 0.663934 & 0.680328 & 0.696721 & 0.704918 & 0.721311 & 0.721311 & \\
\hline
\end{tabular}

Table 15: Cumulative Match Curve For Inter-Trial Randomization on Nose Length Modifications for LDA

Table 15 shows the LDA, Inter-Trial Randomization for the nose length modifications, similar to Table 13 for Intra-Trial Randomization. Seemingly different than the results in Table 13, the Category groupings are different and the rates between the baseline and the treatment curves are bigger than seen in Table 13. For more discussion on the results, Section 4.3 discusses the InterTrial Randomization for both width and length modifications. 
The next set of graphs are for the CMC scores for the other two facial recognition algorithms, PCA and LBPH. First the Intra-Trial Randomization Width Trials will be presented first; followed by the nose length modifications. Next the Inter-Trial Randomization nose width, then nose length, modifications will be shown.

\begin{tabular}{|c|c|c|c|c|c|c|c|c|c|}
\hline trial & R1 Rate & R2 Rate & R3 Rate & R4 Rate & R5 Rate & R6 Rate & R7 Rate & R8 Rate & R9 Rate \\
\hline$-100 \% b$ & 0.601562 & 0.648438 & 0.679688 & 0.710938 & 0.726562 & 0.75 & 0.765625 & 0.789062 & 0.796875 \\
\hline$-100 \% t$ & 0.601562 & 0.664062 & 0.703125 & 0.71875 & 0.726562 & 0.742188 & 0.78125 & 0.804688 & 0.804688 \\
\hline$-95 \% b$ & 0.582677 & 0.622047 & 0.653543 & 0.677165 & 0.685039 & 0.692913 & 0.700787 & 0.700787 & 0.716535 \\
\hline$-95 \% t$ & 0.582677 & 0.637795 & 0.645669 & 0.669291 & 0.692913 & 0.700787 & 0.700787 & 0.708661 & 0.716535 \\
\hline$-90 \% b$ & 0.544 & 0.584 & 0.64 & 0.672 & 0.72 & 0.76 & 0.776 & 0.792 & 0.824 \\
\hline$-90 \% t$ & 0.56 & 0.6 & 0.632 & 0.688 & 0.72 & 0.768 & 0.8 & 0.816 & 0.84 \\
\hline$-85 \% b$ & 0.614754 & 0.663934 & 0.729508 & 0.754098 & 0.762295 & 0.770492 & 0.778689 & 0.795082 & 0.795082 \\
\hline$-85 \% t$ & 0.590164 & 0.663934 & 0.680328 & 0.729508 & 0.770492 & 0.795082 & 0.803279 & 0.803279 & 0.803279 \\
\hline$-80 \% b$ & 0.639344 & 0.721311 & 0.795082 & 0.827869 & 0.836066 & 0.844262 & 0.844262 & 0.844262 & 0.844262 \\
\hline$-80 \% t$ & 0.655738 & 0.729508 & 0.778689 & 0.778689 & 0.795082 & 0.819672 & 0.827869 & 0.827869 & 0.827869 \\
\hline$-75 \% b$ & 0.590164 & 0.631148 & 0.688525 & 0.696721 & 0.721311 & 0.729508 & 0.737705 & 0.762295 & 0.778689 \\
\hline$-75 \% t$ & 0.57377 & 0.647541 & 0.704918 & 0.713115 & 0.721311 & 0.729508 & 0.737705 & 0.737705 & 0.754098 \\
\hline$-70 \% b$ & 0.592 & 0.664 & 0.68 & 0.728 & 0.776 & 0.776 & 0.792 & 0.792 & 0.8 \\
\hline$-70 \% t$ & 0.576 & 0.64 & 0.72 & 0.736 & 0.784 & 0.8 & 0.832 & 0.832 & 0.832 \\
\hline$-65 \% b$ & 0.590551 & 0.645669 & 0.685039 & 0.716535 & 0.748031 & 0.771654 & 0.779528 & 0.779528 & 0.811024 \\
\hline$-65 \% t$ & 0.582677 & 0.669291 & 0.708661 & 0.740157 & 0.76378 & 0.771654 & 0.779528 & 0.811024 & 0.826772 \\
\hline$-60 \% b$ & 0.595238 & 0.666667 & 0.714286 & 0.730159 & 0.753968 & 0.769841 & 0.785714 & 0.785714 & 0.785714 \\
\hline$-60 \% t$ & 0.579365 & 0.650794 & 0.706349 & 0.746032 & 0.753968 & 0.769841 & 0.785714 & 0.793651 & 0.801587 \\
\hline$-55 \% b$ & 0.551181 & 0.614173 & 0.614173 & 0.622047 & 0.629921 & 0.637795 & 0.645669 & 0.645669 & 0.661417 \\
\hline$-55 \% t$ & 0.574803 & 0.653543 & 0.669291 & 0.669291 & 0.669291 & 0.677165 & 0.677165 & 0.677165 & 0.677165 \\
\hline$-50 \% b$ & 0.559055 & 0.606299 & 0.629921 & 0.692913 & 0.740157 & 0.755906 & 0.771654 & 0.779528 & 0.795276 \\
\hline$-50 \% t$ & 0.622047 & 0.677165 & 0.724409 & 0.732283 & 0.732283 & 0.76378 & 0.771654 & 0.787402 & 0.80315 \\
\hline
\end{tabular}




\begin{tabular}{|c|c|c|c|c|c|c|c|c|c|}
\hline$-45 \% b$ & 0.566929 & 0.614173 & 0.629921 & 0.637795 & 0.645669 & 0.661417 & 0.692913 & 0.708661 & 0.716535 \\
\hline$-45 \% t$ & 0.582677 & 0.637795 & 0.645669 & 0.661417 & 0.677165 & 0.685039 & 0.685039 & 0.685039 & 0.692913 \\
\hline$-40 \% b$ & 0.563492 & 0.619048 & 0.674603 & 0.722222 & 0.738095 & 0.746032 & 0.761905 & 0.769841 & 0.801587 \\
\hline$-40 \% t$ & 0.547619 & 0.619048 & 0.666667 & 0.68254 & 0.714286 & 0.738095 & 0.753968 & 0.769841 & 0.793651 \\
\hline$-35 \% b$ & 0.653226 & 0.693548 & 0.741935 & 0.758065 & 0.790323 & 0.790323 & 0.798387 & 0.822581 & 0.822581 \\
\hline$-35 \% t$ & 0.637097 & 0.701613 & 0.741935 & 0.766129 & 0.774194 & 0.790323 & 0.806452 & 0.822581 & 0.846774 \\
\hline$-30 \% b$ & 0.563492 & 0.626984 & 0.68254 & 0.706349 & 0.730159 & 0.753968 & 0.769841 & 0.769841 & 0.769841 \\
\hline$-30 \% t$ & 0.563492 & 0.68254 & 0.690476 & 0.706349 & 0.714286 & 0.738095 & 0.753968 & 0.753968 & 0.769841 \\
\hline$-25 \% b$ & 0.616 & 0.656 & 0.696 & 0.72 & 0.72 & 0.752 & 0.752 & 0.752 & 0.76 \\
\hline$-25 \% t$ & 0.608 & 0.656 & 0.704 & 0.704 & 0.728 & 0.76 & 0.76 & 0.76 & 0.776 \\
\hline$-20 \% b$ & 0.621212 & 0.719697 & 0.75 & 0.772727 & 0.795455 & 0.795455 & 0.795455 & 0.818182 & 0.818182 \\
\hline$-20 \% t$ & 0.674242 & 0.719697 & 0.742424 & 0.765152 & 0.780303 & 0.787879 & 0.787879 & 0.80303 & 0.825758 \\
\hline$-15 \% b$ & 0.620155 & 0.666667 & 0.697674 & 0.713178 & 0.744186 & 0.767442 & 0.775194 & 0.782946 & 0.782946 \\
\hline$-15 \% t$ & 0.604651 & 0.666667 & 0.705426 & 0.744186 & 0.775194 & 0.775194 & 0.782946 & 0.790698 & 0.790698 \\
\hline$-10 \% b$ & 0.522727 & 0.575758 & 0.606061 & 0.628788 & 0.643939 & 0.666667 & 0.689394 & 0.712121 & 0.719697 \\
\hline$-10 \% t$ & 0.530303 & 0.583333 & 0.621212 & 0.651515 & 0.659091 & 0.659091 & 0.674242 & 0.704545 & 0.719697 \\
\hline$-5 \% b$ & 0.543307 & 0.590551 & 0.629921 & 0.645669 & 0.692913 & 0.692913 & 0.700787 & 0.716535 & 0.716535 \\
\hline$-5 \% t$ & 0.535433 & 0.590551 & 0.614173 & 0.622047 & 0.661417 & 0.661417 & 0.677165 & 0.700787 & 0.716535 \\
\hline $5 \% b$ & 0.607692 & 0.623077 & 0.684615 & 0.692308 & 0.715385 & 0.723077 & 0.730769 & 0.730769 & 0.738462 \\
\hline $5 \% \mathrm{t}$ & 0.6 & 0.623077 & 0.669231 & 0.692308 & 0.723077 & 0.730769 & 0.738462 & 0.738462 & 0.738462 \\
\hline $10 \% \mathrm{~b}$ & 0.650794 & 0.65873 & 0.698413 & 0.722222 & 0.738095 & 0.753968 & 0.753968 & 0.761905 & 0.761905 \\
\hline $10 \% \mathrm{t}$ & 0.595238 & 0.65873 & 0.698413 & 0.753968 & 0.753968 & 0.753968 & 0.753968 & 0.769841 & 0.777778 \\
\hline $15 \% b$ & 0.633333 & 0.741667 & 0.783333 & 0.816667 & 0.825 & 0.85 & 0.85 & 0.85 & 0.858333 \\
\hline $15 \% t$ & 0.633333 & 0.716667 & 0.783333 & 0.825 & 0.833333 & 0.85 & 0.85 & 0.858333 & 0.866667 \\
\hline $20 \% b$ & 0.521739 & 0.6 & 0.626087 & 0.626087 & 0.634783 & 0.643478 & 0.652174 & 0.669565 & 0.678261 \\
\hline $20 \% t$ & 0.504348 & 0.530435 & 0.556522 & 0.582609 & 0.591304 & 0.626087 & 0.634783 & 0.634783 & 0.652174 \\
\hline
\end{tabular}




\begin{tabular}{|c|c|c|c|c|c|c|c|c|c|}
\hline $25 \% b$ & 0.5 & 0.614035 & 0.666667 & 0.675439 & 0.675439 & 0.684211 & 0.719298 & 0.736842 & 0.754386 \\
\hline $25 \% \mathrm{t}$ & 0.517544 & 0.605263 & 0.649123 & 0.675439 & 0.684211 & 0.710526 & 0.710526 & 0.72807 & 0.763158 \\
\hline $30 \% b$ & 0.513761 & 0.559633 & 0.59633 & 0.605505 & 0.614679 & 0.623853 & 0.651376 & 0.66055 & 0.66055 \\
\hline $30 \% t$ & 0.53211 & 0.568807 & 0.59633 & 0.605505 & 0.623853 & 0.623853 & 0.66055 & 0.669725 & 0.669725 \\
\hline $35 \% b$ & 0.575221 & 0.60177 & 0.654867 & 0.663717 & 0.690265 & 0.699115 & 0.707965 & 0.725664 & 0.725664 \\
\hline $35 \% t$ & 0.548673 & 0.60177 & 0.619469 & 0.637168 & 0.637168 & 0.672566 & 0.716814 & 0.725664 & 0.743363 \\
\hline $40 \% b$ & 0.561905 & 0.609524 & 0.657143 & 0.695238 & 0.714286 & 0.72381 & 0.742857 & 0.761905 & 0.790476 \\
\hline $40 \% t$ & 0.561905 & 0.628571 & 0.67619 & 0.704762 & 0.714286 & 0.714286 & 0.72381 & 0.752381 & 0.771429 \\
\hline $45 \% b$ & 0.66055 & 0.724771 & 0.724771 & 0.724771 & 0.743119 & 0.752294 & 0.752294 & 0.752294 & 0.761468 \\
\hline $45 \% t$ & 0.587156 & 0.678899 & 0.706422 & 0.715596 & 0.715596 & 0.724771 & 0.743119 & 0.743119 & 0.743119 \\
\hline $50 \% b$ & 0.5625 & 0.625 & 0.651786 & 0.6875 & 0.714286 & 0.758929 & 0.758929 & 0.767857 & 0.767857 \\
\hline $50 \% t$ & 0.517857 & 0.625 & 0.669643 & 0.705357 & 0.714286 & 0.741071 & 0.75 & 0.776786 & 0.776786 \\
\hline $55 \% b$ & 0.705882 & 0.784314 & 0.823529 & 0.833333 & 0.843137 & 0.862745 & 0.862745 & 0.862745 & 0.862745 \\
\hline $55 \% \mathrm{t}$ & 0.745098 & 0.784314 & 0.794118 & 0.813725 & 0.843137 & 0.843137 & 0.872549 & 0.872549 & 0.882353 \\
\hline $60 \% b$ & 0.585106 & 0.638298 & 0.691489 & 0.734043 & 0.755319 & 0.787234 & 0.797872 & 0.797872 & 0.829787 \\
\hline $60 \% t$ & 0.56383 & 0.617021 & 0.670213 & 0.680851 & 0.723404 & 0.755319 & 0.808511 & 0.840426 & 0.861702 \\
\hline $65 \% b$ & 0.517647 & 0.611765 & 0.635294 & 0.658824 & 0.694118 & 0.694118 & 0.694118 & 0.705882 & 0.705882 \\
\hline $65 \% \mathrm{t}$ & 0.529412 & 0.564706 & 0.611765 & 0.658824 & 0.670588 & 0.670588 & 0.670588 & 0.670588 & 0.682353 \\
\hline $70 \% b$ & 0.674157 & 0.707865 & 0.741573 & 0.764045 & 0.764045 & 0.808989 & 0.808989 & 0.831461 & 0.842697 \\
\hline $70 \% t$ & 0.606742 & 0.640449 & 0.662921 & 0.707865 & 0.719101 & 0.752809 & 0.775281 & 0.786517 & 0.797753 \\
\hline $75 \% b$ & 0.539474 & 0.592105 & 0.644737 & 0.671053 & 0.697368 & 0.710526 & 0.723684 & 0.723684 & 0.736842 \\
\hline $75 \% t$ & 0.513158 & 0.592105 & 0.605263 & 0.631579 & 0.684211 & 0.697368 & 0.723684 & 0.736842 & 0.736842 \\
\hline $80 \% b$ & 0.574713 & 0.597701 & 0.609195 & 0.632184 & 0.666667 & 0.689655 & 0.701149 & 0.712644 & 0.724138 \\
\hline $80 \% t$ & 0.563218 & 0.609195 & 0.609195 & 0.643678 & 0.643678 & 0.678161 & 0.701149 & 0.701149 & 0.747126 \\
\hline $85 \% b$ & 0.666667 & 0.736111 & 0.805556 & 0.819444 & 0.819444 & 0.819444 & 0.833333 & 0.833333 & 0.847222 \\
\hline $85 \% \mathrm{t}$ & 0.541667 & 0.638889 & 0.694444 & 0.708333 & 0.763889 & 0.805556 & 0.819444 & 0.861111 & 0.861111 \\
\hline
\end{tabular}




\begin{tabular}{|l|r|r|l|l|l|l|l|l|l|}
\hline $\mathbf{9 0 \% b}$ & 0.534483 & 0.637931 & 0.655172 & 0.689655 & 0.724138 & 0.775862 & 0.793103 & 0.793103 & 0.793103 \\
\hline $\mathbf{9 0 \% t}$ & 0.517241 & 0.568966 & 0.637931 & 0.637931 & 0.655172 & 0.689655 & 0.706897 & 0.724138 & 0.758621 \\
\hline $\mathbf{9 5 \% b}$ & 0.538462 & 0.576923 & 0.634615 & 0.673077 & 0.730769 & 0.75 & 0.788462 & 0.807692 & 0.807692 \\
\hline $\mathbf{9 5 \% t}$ & 0.365385 & 0.423077 & 0.480769 & 0.519231 & 0.519231 & 0.538462 & 0.576923 & 0.576923 & 0.596154 \\
\hline $\mathbf{1 0 0 \% b}$ & 0.75 & 0.795455 & 0.818182 & 0.818182 & 0.840909 & 0.863636 & 0.863636 & 0.863636 & 0.886364 \\
\hline $\mathbf{1 0 0 \% t}$ & 0.522727 & 0.568182 & 0.613636 & 0.659091 & 0.681818 & 0.727273 & 0.795455 & 0.818182 & 0.818182 \\
\hline
\end{tabular}

Table 16: Cumulative Match Curve For Intra-Trial Randomization on Nose Width Modifications for PCA.

\begin{tabular}{|c|c|c|c|c|c|c|c|c|c|}
\hline Trials & R1 Rate & R2 Rate & R3 Rate & R4 Rate & R5 Rate & R6 Rate & R7 Rate & R8 Rate & R9 Rate \\
\hline$-20 \% b$ & 0.54918 & 0.598361 & 0.680328 & 0.737705 & 0.754098 & 0.778689 & 0.786885 & 0.786885 & 0.786885 \\
\hline$-20 \% t$ & 0.52459 & 0.581967 & 0.647541 & 0.688525 & 0.745902 & 0.778689 & 0.795082 & 0.795082 & 0.795082 \\
\hline$-15 \% b$ & 0.589147 & 0.666667 & 0.682171 & 0.713178 & 0.751938 & 0.782946 & 0.79845 & 0.813953 & 0.821705 \\
\hline$-15 \% t$ & 0.596899 & 0.651163 & 0.697674 & 0.705426 & 0.72093 & 0.751938 & 0.767442 & 0.79845 & 0.813953 \\
\hline$-10 \% b$ & 0.601626 & 0.634146 & 0.666667 & 0.699187 & 0.715447 & 0.723577 & 0.747967 & 0.764228 & 0.772358 \\
\hline$-10 \% t$ & 0.593496 & 0.650407 & 0.674797 & 0.699187 & 0.723577 & 0.731707 & 0.764228 & 0.772358 & 0.780488 \\
\hline$-5 \% b$ & 0.533835 & 0.62406 & 0.646617 & 0.669173 & 0.699248 & 0.721805 & 0.721805 & 0.75188 & 0.774436 \\
\hline$-5 \% t$ & 0.541353 & 0.616541 & 0.639098 & 0.661654 & 0.699248 & 0.721805 & 0.729323 & 0.75188 & 0.774436 \\
\hline $5 \% b$ & 0.59375 & 0.679688 & 0.695312 & 0.734375 & 0.757812 & 0.78125 & 0.789062 & 0.796875 & 0.8125 \\
\hline $5 \% \mathrm{t}$ & 0.5625 & 0.664062 & 0.726562 & 0.757812 & 0.773438 & 0.78125 & 0.789062 & 0.804688 & 0.8125 \\
\hline $10 \% b$ & 0.542636 & 0.581395 & 0.620155 & 0.674419 & 0.697674 & 0.713178 & 0.736434 & 0.736434 & 0.744186 \\
\hline $10 \% \mathrm{t}$ & 0.573643 & 0.604651 & 0.643411 & 0.697674 & 0.72093 & 0.736434 & 0.751938 & 0.75969 & 0.75969 \\
\hline $15 \% b$ & 0.608 & 0.656 & 0.688 & 0.728 & 0.76 & 0.784 & 0.792 & 0.792 & 0.848 \\
\hline $15 \% \mathrm{t}$ & 0.592 & 0.64 & 0.72 & 0.744 & 0.776 & 0.784 & 0.792 & 0.8 & 0.808 \\
\hline $20 \% b$ & 0.575 & 0.65 & 0.7 & 0.733333 & 0.75 & 0.75 & 0.75 & 0.758333 & 0.766667 \\
\hline $20 \% \mathrm{t}$ & 0.6 & 0.683333 & 0.716667 & 0.741667 & 0.775 & 0.775 & 0.775 & 0.775 & 0.775 \\
\hline
\end{tabular}




\begin{tabular}{|c|c|c|c|c|c|c|c|c|c|}
\hline trial & R1 Rate & R2 Rate & R3 Rate & R4 Rate & R5 Rate & R6 Rate & R7 Rate & R8 Rate & R9 Rate \\
\hline$-100 \% b$ & 0.578125 & 0.65625 & 0.703125 & 0.734375 & 0.757812 & 0.773438 & 0.78125 & 0.789062 & 0.789062 \\
\hline$-100 \% t$ & 0.609375 & 0.679688 & 0.703125 & 0.734375 & 0.75 & 0.75 & 0.765625 & 0.773438 & 0.773438 \\
\hline$-95 \% b$ & 0.570312 & 0.65625 & 0.703125 & 0.734375 & 0.757812 & 0.773438 & 0.78125 & 0.789062 & 0.789062 \\
\hline$-95 \% t$ & 0.59375 & 0.65625 & 0.695312 & 0.726562 & 0.742188 & 0.742188 & 0.765625 & 0.773438 & 0.78125 \\
\hline$-90 \% b$ & 0.584 & 0.656 & 0.704 & 0.736 & 0.76 & 0.776 & 0.784 & 0.792 & 0.792 \\
\hline$-90 \% t$ & 0.584 & 0.64 & 0.68 & 0.72 & 0.736 & 0.76 & 0.776 & 0.792 & 0.792 \\
\hline$-85 \% b$ & 0.568 & 0.648 & 0.696 & 0.728 & 0.752 & 0.768 & 0.776 & 0.784 & 0.784 \\
\hline$-85 \% t$ & 0.576 & 0.632 & 0.672 & 0.688 & 0.696 & 0.712 & 0.744 & 0.752 & 0.76 \\
\hline$-80 \% b$ & 0.564516 & 0.645161 & 0.693548 & 0.725806 & 0.75 & 0.766129 & 0.774194 & 0.782258 & 0.782258 \\
\hline$-80 \% t$ & 0.556452 & 0.620968 & 0.669355 & 0.701613 & 0.717742 & 0.717742 & 0.725806 & 0.766129 & 0.774194 \\
\hline$-75 \% b$ & 0.568 & 0.648 & 0.696 & 0.728 & 0.752 & 0.768 & 0.776 & 0.784 & 0.784 \\
\hline$-75 \% t$ & 0.576 & 0.648 & 0.68 & 0.704 & 0.736 & 0.744 & 0.744 & 0.76 & 0.76 \\
\hline$-70 \% b$ & 0.571429 & 0.65873 & 0.706349 & 0.738095 & 0.761905 & 0.777778 & 0.785714 & 0.793651 & 0.793651 \\
\hline$-70 \% t$ & 0.587302 & 0.65873 & 0.698413 & 0.730159 & 0.746032 & 0.753968 & 0.769841 & 0.785714 & 0.793651 \\
\hline$-65 \% b$ & 0.576 & 0.664 & 0.712 & 0.744 & 0.768 & 0.784 & 0.792 & 0.8 & 0.8 \\
\hline$-65 \% t$ & 0.576 & 0.64 & 0.696 & 0.72 & 0.728 & 0.744 & 0.744 & 0.76 & 0.76 \\
\hline$-60 \% b$ & 0.579365 & 0.666667 & 0.714286 & 0.746032 & 0.769841 & 0.785714 & 0.793651 & 0.801587 & 0.801587 \\
\hline$-60 \% t$ & 0.603175 & 0.650794 & 0.706349 & 0.730159 & 0.746032 & 0.761905 & 0.769841 & 0.793651 & 0.793651 \\
\hline$-55 \% b$ & 0.580645 & 0.66129 & 0.709677 & 0.741935 & 0.766129 & 0.782258 & 0.790323 & 0.798387 & 0.798387 \\
\hline$-55 \% t$ & 0.620968 & 0.669355 & 0.717742 & 0.75 & 0.774194 & 0.774194 & 0.782258 & 0.806452 & 0.806452 \\
\hline$-50 \% b$ & 0.568 & 0.648 & 0.696 & 0.728 & 0.752 & 0.768 & 0.776 & 0.784 & 0.784 \\
\hline$-50 \% t$ & 0.592 & 0.664 & 0.704 & 0.728 & 0.728 & 0.744 & 0.76 & 0.776 & 0.776 \\
\hline$-45 \% b$ & 0.576 & 0.656 & 0.704 & 0.736 & 0.76 & 0.776 & 0.784 & 0.792 & 0.792 \\
\hline$-45 \% t$ & 0.616 & 0.68 & 0.712 & 0.728 & 0.736 & 0.744 & 0.752 & 0.776 & 0.792 \\
\hline$-40 \% b$ & 0.571429 & 0.650794 & 0.698413 & 0.738095 & 0.761905 & 0.777778 & 0.785714 & 0.793651 & 0.793651 \\
\hline
\end{tabular}




\begin{tabular}{|c|c|c|c|c|c|c|c|c|c|}
\hline$-40 \% t$ & 0.579365 & 0.650794 & 0.714286 & 0.730159 & 0.738095 & 0.738095 & 0.746032 & 0.746032 & 0.753968 \\
\hline$-35 \% b$ & 0.574803 & 0.653543 & 0.700787 & 0.740157 & 0.76378 & 0.779528 & 0.787402 & 0.795276 & 0.795276 \\
\hline$-35 \% t$ & 0.574803 & 0.661417 & 0.692913 & 0.716535 & 0.732283 & 0.740157 & 0.76378 & 0.771654 & 0.787402 \\
\hline$-30 \% b$ & 0.574803 & 0.653543 & 0.700787 & 0.740157 & 0.76378 & 0.779528 & 0.787402 & 0.795276 & 0.795276 \\
\hline$-30 \% t$ & 0.559055 & 0.629921 & 0.692913 & 0.740157 & 0.740157 & 0.755906 & 0.771654 & 0.771654 & 0.779528 \\
\hline$-25 \% b$ & 570312 & 0.640625 & 0.6875 & 0.726562 & 0.75 & 0.765625 & 0.773438 & 0.78125 & 0.78125 \\
\hline$-25 \% t$ & 0.554688 & 0.648438 & 0.703125 & 0.75 & 0.757812 & 0.765625 & 0.773438 & 0.78125 & 0.78125 \\
\hline$-20 \% b$ & 0.55814 & 0.643411 & 0.689922 & 0.728682 & 0.751938 & 0.767442 & 0.775194 & 0.782946 & 0.782946 \\
\hline$-20 \% t$ & 0.596899 & 0.651163 & 0.682171 & 0.72093 & 0.736434 & 0.767442 & 0.775194 & 0.775194 & 0.782946 \\
\hline$-15 \% b$ & 0.5625 & 0.648438 & 0.695312 & 0.734375 & 0.757812 & 0.773438 & 0.78125 & 0.789062 & 0.789062 \\
\hline$-15 \% t$ & 0.585938 & 0.640625 & 0.6875 & 0.71875 & 0.734375 & 0.757812 & 0.765625 & 0.773438 & 0.78125 \\
\hline$-10 \% b$ & 55814 & 0.643411 & 0.689922 & 0.728682 & 0.751938 & 0.767442 & 0.775194 & 0.782946 & 0.782946 \\
\hline$-10 \% t$ & 73643 & 0.627907 & 0.682171 & 0.713178 & 0.728682 & 0.751938 & 0.75969 & 0.775194 & 0.775194 \\
\hline$-5 \% b$ & 0.566929 & 0.645669 & 0.692913 & 0.732283 & 0.755906 & 0.771654 & 0.779528 & 0.787402 & 0.787402 \\
\hline$-5 \% \mathrm{t}$ & 0.598425 & 0.645669 & 0.677165 & 0.708661 & 0.724409 & 0.755906 & 0.76378 & 0.779528 & 0.779528 \\
\hline $5 \% \mathrm{~b}$ & 0.581395 & 0.658915 & 0.705426 & 0.736434 & 0.75969 & 0.775194 & 0.782946 & 0.790698 & 0.790698 \\
\hline $5 \% t$ & 0.581395 & 0.658915 & 0.682171 & 0.713178 & 0.728682 & 0.75969 & 0.775194 & 0.790698 & 0.79845 \\
\hline $10 \% \mathrm{~b}$ & 0.578125 & 0.65625 & 0.703125 & 0.734375 & 0.757812 & 0.773438 & 0.78125 & 0.789062 & 0.789062 \\
\hline $10 \% \mathrm{t}$ & 59375 & 0.648438 & 0.679688 & 0.71875 & 0.726562 & 0.757812 & 0.773438 & 0.789062 & 0.796875 \\
\hline $15 \% b$ & 0.581967 & 0.655738 & 0.704918 & 0.737705 & 0.762295 & 0.778689 & 0.786885 & 0.795082 & 0.795082 \\
\hline $15 \% \mathrm{t}$ & 0.590164 & 0.622951 & 0.655738 & 0.688525 & 0.713115 & 0.745902 & 0.770492 & 0.770492 & 0.786885 \\
\hline $20 \% b$ & 0.589744 & 0.649573 & 0.700855 & 0.735043 & 0.752137 & 0.760684 & 0.769231 & 0.777778 & 0.777778 \\
\hline $20 \% t$ & 0.623932 & 0.649573 & 0.700855 & 0.700855 & 0.717949 & 0.726496 & 0.752137 & 0.760684 & 0.777778 \\
\hline $25 \% b$ & 0.578947 & 0.640351 & 0.692982 & 0.72807 & 0.745614 & 0.763158 & 0.77193 & 0.780702 & 0.780702 \\
\hline $25 \% \mathrm{t}$ & 0.578947 & 0.614035 & 0.666667 & 0.675439 & 0.719298 & 0.719298 & 0.736842 & 0.763158 & 0.780702 \\
\hline $30 \% \mathrm{~b}$ & 0.60177 & 0.672566 & 0.716814 & 0.752212 & 0.778761 & 0.787611 & 0.79646 & 0.80531 & 0.80531 \\
\hline
\end{tabular}




\begin{tabular}{|c|c|c|c|c|c|c|c|c|c|}
\hline $30 \% t$ & 0.610619 & 0.663717 & 0.699115 & 0.725664 & 0.743363 & 0.769912 & 0.769912 & 0.79646 & 0.79646 \\
\hline $35 \% b$ & 0.594595 & 0.657658 & 0.702703 & 0.738739 & 0.756757 & 0.765766 & 0.774775 & 0.783784 & 0.783784 \\
\hline $35 \% \mathrm{t}$ & 0.63964 & 0.711712 & 0.738739 & 0.756757 & 0.756757 & 0.774775 & 0.774775 & 0.783784 & 0.783784 \\
\hline $40 \% b$ & 0.603774 & 0.669811 & 0.716981 & 0.754717 & 0.764151 & 0.773585 & 0.783019 & 0.792453 & 0.792453 \\
\hline $40 \% t$ & 0.650943 & 0.707547 & 0.745283 & 0.764151 & 0.773585 & 0.811321 & 0.811321 & 0.811321 & 0.820755 \\
\hline $45 \% b$ & 0.617647 & 0.686275 & 0.735294 & 0.754902 & 0.764706 & 0.77451 & 0.784314 & 0.794118 & 0.794118 \\
\hline $45 \% \mathrm{t}$ & 0.627451 & 0.686275 & 0.715686 & 0.745098 & 0.754902 & 0.77451 & 0.77451 & 0.784314 & 0.803922 \\
\hline $50 \% b$ & 0.617647 & 0.696078 & 0.735294 & 0.764706 & 0.77451 & 0.784314 & 0.794118 & 0.803922 & 0.803922 \\
\hline $50 \% t$ & 0.666667 & 0.72549 & 0.735294 & 0.754902 & 0.77451 & 0.77451 & 0.784314 & 0.794118 & 0.813725 \\
\hline $55 \% b$ & 0.622449 & 0.683673 & 0.744898 & 0.77551 & 0.77551 & 0.785714 & 0.795918 & 0.806122 & 0.806122 \\
\hline $55 \% \mathrm{t}$ & 0.591837 & 0.704082 & 0.714286 & 0.72449 & 0.734694 & 0.755102 & 0.765306 & 0.77551 & 0.77551 \\
\hline $60 \% b$ & 0.642105 & 0.715789 & 0.757895 & 0.768421 & 0.778947 & 0.789474 & 0.8 & 0.810526 & 0.810526 \\
\hline $60 \% t$ & 0.673684 & 0.705263 & 0.726316 & 0.736842 & 0.747368 & 0.757895 & 0.757895 & 0.768421 & 0.778947 \\
\hline $65 \% b$ & 0.630435 & 0.695652 & 0.73913 & 0.75 & 0.76087 & 0.771739 & 0.782609 & 0.793478 & 0.793478 \\
\hline $65 \% \mathrm{t}$ & 0.586957 & 0.695652 & 0.717391 & 0.75 & 0.76087 & 0.771739 & 0.771739 & 0.793478 & 0.793478 \\
\hline $70 \% b$ & 0.644444 & 0.688889 & 0.733333 & 0.755556 & 0.766667 & 0.777778 & 0.788889 & 0.8 & 0.8 \\
\hline $70 \% t$ & 0.6 & 0.677778 & 0.7 & 0.733333 & 0.733333 & 0.744444 & 0.755556 & 0.755556 & 0.777778 \\
\hline $75 \% b$ & 0.60241 & 0.662651 & 0.710843 & 0.73494 & 0.73494 & 0.746988 & 0.759036 & 0.759036 & 0.759036 \\
\hline $75 \% t$ & 0.614458 & 0.686747 & 0.710843 & 0.73494 & 0.73494 & 0.746988 & 0.759036 & 0.759036 & 0.759036 \\
\hline $80 \% b$ & 0.64 & 0.706667 & 0.746667 & 0.76 & 0.76 & 0.773333 & 0.786667 & 0.786667 & 0.786667 \\
\hline $80 \% t$ & 0.56 & 0.64 & 0.693333 & 0.706667 & 0.746667 & 0.746667 & 0.746667 & 0.76 & 0.76 \\
\hline $85 \% b$ & 0.605634 & 0.676056 & 0.704225 & 0.71831 & 0.71831 & 0.732394 & 0.746479 & 0.746479 & 0.746479 \\
\hline $85 \% t$ & 0.591549 & 0.647887 & 0.676056 & 0.704225 & 0.746479 & 0.746479 & 0.760563 & 0.760563 & 0.760563 \\
\hline $90 \% b$ & 0.55 & 0.633333 & 0.666667 & 0.683333 & 0.683333 & 0.683333 & 0.7 & 0.7 & 0.7 \\
\hline $90 \% \mathrm{t}$ & 0.55 & 0.6 & 0.6 & 0.616667 & 0.633333 & 0.65 & 0.666667 & 0.7 & 0.716667 \\
\hline $95 \% b$ & 0.64 & 0.7 & 0.72 & 0.72 & 0.72 & 0.72 & 0.74 & 0.74 & 0.74 \\
\hline
\end{tabular}




\begin{tabular}{|r|r|r|r|r|r|r|r|r|r|r|}
\hline $\mathbf{9 5 \% t}$ & 0.54 & 0.56 & 0.6 & 0.64 & 0.7 & 0.7 & 0.72 & 0.72 & 0.74 \\
\hline $\mathbf{1 0 0 \% b}$ & 0.638298 & 0.702128 & 0.723404 & 0.723404 & 0.723404 & 0.723404 & 0.744681 & 0.744681 & 0.744681 \\
\hline $\mathbf{1 0 0 \%}$ & 0.617021 & 0.638298 & 0.702128 & 0.744681 & 0.744681 & 0.744681 & 0.765957 & 0.787234 & 0.787234 \\
\hline & $\begin{array}{l}\text { Table 18: Cumulative Match Curve For Inter-Trial Randomization on Nose } \\
\text { Width Modifications for PCA }\end{array}$ \\
\hline
\end{tabular}

\begin{tabular}{|c|c|c|c|c|c|c|c|c|c|}
\hline trial & R1 Rate & R2 Rate & R3 Rate & R4 Rate & R5 Rate & R6 Rate & R7 Rate & R8 Rate & R9 Rate \\
\hline$-20 \% b$ & 0.580645 & 0.669355 & 0.717742 & 0.75 & 0.774194 & 0.790323 & 0.798387 & 0.806452 & 0.806452 \\
\hline$-20 \% t$ & 0.596774 & 0.701613 & 0.717742 & 0.733871 & 0.766129 & 0.798387 & 0.806452 & 0.806452 & 0.806452 \\
\hline$-15 \% b$ & 0.571429 & 0.650794 & 0.698413 & 0.730159 & 0.753968 & 0.769841 & 0.777778 & 0.785714 & 0.785714 \\
\hline$-15 \% t$ & 0.579365 & 0.65873 & 0.690476 & 0.722222 & 0.746032 & 0.785714 & 0.785714 & 0.785714 & 0.785714 \\
\hline$-10 \% b$ & 0.564885 & 0.641221 & 0.687023 & 0.725191 & 0.748092 & 0.763359 & 0.770992 & 0.778626 & 0.778626 \\
\hline$-10 \% t$ & 0.603053 & 0.664122 & 0.694656 & 0.732824 & 0.748092 & 0.778626 & 0.78626 & 0.78626 & 0.78626 \\
\hline$-5 \% b$ & 0.56391 & 0.639098 & 0.684211 & 0.721805 & 0.744361 & 0.759398 & 0.766917 & 0.774436 & 0.774436 \\
\hline$-5 \% \mathrm{t}$ & 0.578947 & 0.646617 & 0.691729 & 0.729323 & 0.744361 & 0.766917 & 0.774436 & 0.774436 & 0.774436 \\
\hline $5 \% b$ & 0.566929 & 0.653543 & 0.700787 & 0.740157 & 0.76378 & 0.771654 & 0.779528 & 0.787402 & 0.787402 \\
\hline $5 \% \mathrm{t}$ & 0.566929 & 0.661417 & 0.716535 & 0.740157 & 0.748031 & 0.748031 & 0.748031 & 0.76378 & 0.787402 \\
\hline $10 \% b$ & 0.571429 & 0.65873 & 0.706349 & 0.746032 & 0.769841 & 0.777778 & 0.785714 & 0.793651 & 0.793651 \\
\hline $10 \% t$ & 0.587302 & 0.650794 & 0.722222 & 0.746032 & 0.753968 & 0.753968 & 0.753968 & 0.761905 & 0.769841 \\
\hline $15 \% b$ & 0.564516 & 0.645161 & 0.693548 & 0.733871 & 0.758065 & 0.774194 & 0.782258 & 0.790323 & 0.790323 \\
\hline $15 \% \mathrm{t}$ & 0.564516 & 0.645161 & 0.693548 & 0.717742 & 0.733871 & 0.75 & 0.75 & 0.758065 & 0.774194 \\
\hline $20 \% \mathrm{~b}$ & 0.590164 & 0.663934 & 0.713115 & 0.737705 & 0.762295 & 0.778689 & 0.786885 & 0.795082 & 0.795082 \\
\hline $20 \% t$ & 0.614754 & 0.663934 & 0.713115 & 0.721311 & 0.737705 & 0.737705 & 0.745902 & 0.754098 & 0.754098 \\
\hline
\end{tabular}

\begin{tabular}{|l|l|l|l|l|l|l|l|l|l|}
\hline trial & R1 Rate & R2 Rate & R3 Rate & R4 Rate & R5 Rate & R6 Rate & R7 Rate & R8 Rate & R9 Rate \\
\hline $\mathbf{- 1 0 0 \% b}$ & 0.731707 & 0.764228 & 0.804878 & 0.804878 & 0.813008 & 0.821138 & 0.829268 & 0.837398 & 0.837398 \\
\hline
\end{tabular}




\begin{tabular}{|c|c|c|c|c|c|c|c|c|c|}
\hline$-100 \% t$ & 0.772358 & 0.788618 & 0.813008 & 0.813008 & 0.821138 & 0.829268 & 0.845528 & 0.853659 & 0.853659 \\
\hline$-95 \% b$ & 0.640625 & 0.695312 & 0.734375 & 0.734375 & 0.742188 & 0.742188 & 0.75 & 0.757812 & 0.773438 \\
\hline$-95 \% t$ & 0.632812 & 0.695312 & 0.726562 & 0.757812 & 0.765625 & 0.773438 & 0.773438 & 0.773438 & 0.773438 \\
\hline$-90 \% b$ & 0.672 & 0.728 & 0.752 & 0.768 & 0.776 & 0.776 & 0.776 & 0.792 & 0.8 \\
\hline$-90 \% t$ & 0.688 & 0.712 & 0.744 & 0.776 & 0.776 & 0.8 & 0.808 & 0.808 & 0.816 \\
\hline$-85 \% b$ & 0.634921 & 0.68254 & 0.698413 & 0.722222 & 0.722222 & 0.738095 & 0.746032 & 0.753968 & 0.761905 \\
\hline$-85 \% t$ & 0.619048 & 0.650794 & 0.68254 & 0.746032 & 0.769841 & 0.769841 & 0.785714 & 0.793651 & 0.801587 \\
\hline$-80 \% b$ & 0.75 & 0.774194 & 0.798387 & 0.814516 & 0.814516 & 0.830645 & 0.830645 & 0.83871 & 0.83871 \\
\hline$-80 \% t$ & 0.75 & 0.790323 & 0.798387 & 0.822581 & 0.830645 & 0.830645 & 0.83871 & 0.846774 & 0.846774 \\
\hline$-75 \% b$ & 0.733871 & 0.766129 & 0.790323 & 0.806452 & 0.806452 & 0.814516 & 0.814516 & 0.822581 & 0.83871 \\
\hline$-75 \% t$ & 0.741935 & 0.782258 & 0.798387 & 0.814516 & 0.814516 & 0.830645 & 0.83871 & 0.83871 & 0.83871 \\
\hline$-70 \% b$ & 0.672131 & 0.713115 & 0.737705 & 0.786885 & 0.795082 & 0.795082 & 0.795082 & 0.811475 & 0.811475 \\
\hline$-70 \% t$ & 0.729508 & 0.754098 & 0.762295 & 0.770492 & 0.770492 & 0.803279 & 0.803279 & 0.811475 & 0.819672 \\
\hline$-65 \% b$ & 0.716535 & 0.76378 & 0.779528 & 0.779528 & 0.795276 & 0.80315 & 0.818898 & 0.834646 & 0.834646 \\
\hline$-65 \% t$ & 0.716535 & 0.748031 & 0.787402 & 0.80315 & 0.818898 & 0.818898 & 0.818898 & 0.818898 & 0.834646 \\
\hline$-60 \% b$ & 0.669355 & 0.685484 & 0.693548 & 0.717742 & 0.717742 & 0.725806 & 0.733871 & 0.741935 & 0.75 \\
\hline$-60 \% t$ & 0.653226 & 0.66129 & 0.669355 & 0.677419 & 0.701613 & 0.717742 & 0.717742 & 0.717742 & 0.717742 \\
\hline$-55 \% b$ & 0.692913 & 0.755906 & 0.755906 & 0.771654 & 0.787402 & 0.80315 & 0.80315 & 0.811024 & 0.811024 \\
\hline$-55 \% t$ & 0.732283 & 0.787402 & 0.795276 & 0.80315 & 0.818898 & 0.826772 & 0.850394 & 0.850394 & 0.850394 \\
\hline$-50 \% b$ & 0.778689 & 0.819672 & 0.852459 & 0.868852 & 0.877049 & 0.877049 & 0.877049 & 0.885246 & 0.893443 \\
\hline$-50 \% t$ & 0.778689 & 0.852459 & 0.868852 & 0.877049 & 0.885246 & 0.901639 & 0.901639 & 0.901639 & 0.901639 \\
\hline$-45 \% b$ & 0.546875 & 0.585938 & 0.617188 & 0.640625 & 0.640625 & 0.65625 & 0.65625 & 0.664062 & 0.6875 \\
\hline$-45 \% t$ & 0.5625 & 0.617188 & 0.640625 & 0.671875 & 0.695312 & 0.695312 & 0.703125 & 0.703125 & 0.703125 \\
\hline$-40 \% b$ & 0.740157 & 0.811024 & 0.850394 & 0.858268 & 0.874016 & 0.874016 & 0.874016 & 0.88189 & 0.897638 \\
\hline$-40 \% t$ & 0.732283 & 0.787402 & 0.811024 & 0.818898 & 0.850394 & 0.858268 & 0.858268 & 0.858268 & 0.874016 \\
\hline$-35 \% b$ & 0.587786 & 0.625954 & 0.679389 & 0.694656 & 0.70229 & 0.717557 & 0.732824 & 0.740458 & 0.740458 \\
\hline
\end{tabular}




\begin{tabular}{|c|c|c|c|c|c|c|c|c|c|}
\hline$-35 \% t$ & 0.587786 & 0.671756 & 0.687023 & 0.717557 & 0.717557 & 0.717557 & 0.725191 & 0.725191 & 0.740458 \\
\hline$-30 \% b$ & 0.656489 & 0.694656 & 0.732824 & 0.740458 & 0.748092 & 0.748092 & 0.755725 & 0.778626 & 0.778626 \\
\hline$-30 \% t$ & 0.648855 & 0.70229 & 0.725191 & 0.732824 & 0.755725 & 0.770992 & 0.778626 & 0.801527 & 0.80916 \\
\hline$-25 \% b$ & 0.648 & 0.664 & 0.672 & 0.704 & 0.712 & 0.72 & 0.736 & 0.76 & 0.76 \\
\hline$-25 \% t$ & 0.624 & 0.664 & 0.688 & 0.712 & 0.728 & 0.736 & 0.744 & 0.768 & 0.784 \\
\hline$-20 \% b$ & 0.617188 & 0.648438 & 0.65625 & 0.664062 & 0.671875 & 0.6875 & 0.703125 & 0.71875 & 0.75 \\
\hline$-20 \% t$ & 0.632812 & 0.640625 & 0.671875 & 0.679688 & 0.703125 & 0.710938 & 0.734375 & 0.75 & 0.757812 \\
\hline$-15 \% b$ & 0.72093 & 0.744186 & 0.751938 & 0.775194 & 0.790698 & 0.806202 & 0.806202 & 0.813953 & 0.829457 \\
\hline$-15 \% t$ & 0.728682 & 0.751938 & 0.767442 & 0.775194 & 0.79845 & 0.813953 & 0.813953 & 0.821705 & 0.829457 \\
\hline$-10 \% b$ & 0.646154 & 0.684615 & 0.723077 & 0.738462 & 0.746154 & 0.753846 & 0.753846 & 0.761538 & 0.761538 \\
\hline$-10 \% \mathrm{t}$ & 0.676923 & 0.715385 & 0.723077 & 0.730769 & 0.738462 & 0.753846 & 0.761538 & 0.761538 & 0.761538 \\
\hline$-5 \% b$ & 0.641221 & 0.687023 & 0.70229 & 0.717557 & 0.725191 & 0.740458 & 0.748092 & 0.748092 & 0.755725 \\
\hline$-5 \% t$ & 571756 & 0.694656 & 0.717557 & 0.725191 & 0.732824 & 0.732824 & 0.732824 & 0.755725 & 0.763359 \\
\hline $5 \% b$ & 0.666667 & 0.689922 & 0.736434 & 0.75969 & 0.775194 & 0.790698 & 0.813953 & 0.821705 & 0.829457 \\
\hline $5 \% \mathrm{t}$ & 0.682171 & 0.697674 & 0.744186 & 0.751938 & 0.775194 & 0.790698 & 0.813953 & 0.821705 & 0.821705 \\
\hline $10 \% b$ & 0.715385 & 0.776923 & 0.784615 & 0.784615 & 0.784615 & 0.792308 & 0.792308 & 0.8 & 0.8 \\
\hline $10 \% \mathrm{t}$ & 0.692308 & 0.746154 & 0.769231 & 0.784615 & 0.784615 & 0.8 & 0.815385 & 0.815385 & 0.815385 \\
\hline $15 \% b$ & 0.705882 & 0.798319 & 0.815126 & 0.815126 & 0.823529 & 0.823529 & 0.831933 & 0.840336 & 0.840336 \\
\hline $15 \% \mathrm{t}$ & 0.705882 & 0.764706 & 0.798319 & 0.806723 & 0.815126 & 0.831933 & 0.840336 & 0.840336 & 0.840336 \\
\hline $20 \% b$ & 0.700855 & 0.726496 & 0.74359 & 0.74359 & 0.760684 & 0.786325 & 0.786325 & 0.786325 & 0.794872 \\
\hline $20 \% \mathrm{t}$ & 0.683761 & 0.700855 & 0.717949 & 0.717949 & 0.735043 & 0.752137 & 0.777778 & 0.786325 & 0.786325 \\
\hline $25 \% b$ & 0.685185 & 0.712963 & 0.740741 & 0.75 & 0.759259 & 0.759259 & 0.768519 & 0.787037 & 0.787037 \\
\hline $25 \% \mathrm{t}$ & 0.675926 & 0.731481 & 0.75 & 0.75 & 0.759259 & 0.759259 & 0.759259 & 0.768519 & 0.768519 \\
\hline $30 \% b$ & 0.754545 & 0.781818 & 0.8 & 0.8 & 0.836364 & 0.836364 & 0.854545 & 0.854545 & 0.872727 \\
\hline $30 \% t$ & 0.718182 & 0.8 & 0.8 & 0.836364 & 0.854545 & 0.854545 & 0.854545 & 0.854545 & 0.854545 \\
\hline $35 \% b$ & 0.725664 & 0.778761 & 0.80531 & 0.814159 & 0.823009 & 0.823009 & 0.831858 & 0.831858 & 0.831858 \\
\hline
\end{tabular}




\begin{tabular}{|c|c|c|c|c|c|c|c|c|c|}
\hline $35 \% t$ & 0.707965 & 0.761062 & 0.769912 & 0.79646 & 0.80531 & 0.823009 & 0.831858 & 0.831858 & 0.831858 \\
\hline $40 \% b$ & 0.654545 & 0.690909 & 0.718182 & 0.754545 & 0.763636 & 0.781818 & 0.8 & 0.809091 & 0.827273 \\
\hline $40 \% t$ & 0.645455 & 0.7 & 0.763636 & 0.772727 & 0.781818 & 0.8 & 0.8 & 0.827273 & 0.836364 \\
\hline $45 \% b$ & 0.711538 & 0.769231 & 0.807692 & 0.826923 & 0.836538 & 0.836538 & 0.846154 & 0.846154 & 0.875 \\
\hline $45 \% \mathrm{t}$ & 0.673077 & 0.730769 & 0.769231 & 0.769231 & 0.798077 & 0.817308 & 0.836538 & 0.846154 & 0.855769 \\
\hline $50 \% b$ & 0.647059 & 0.656863 & 0.696078 & 0.72549 & 0.745098 & 0.764706 & 0.794118 & 0.803922 & 0.813725 \\
\hline $50 \% t$ & 0.588235 & 0.647059 & 0.666667 & 0.705882 & 0.72549 & 0.735294 & 0.745098 & 0.745098 & 0.764706 \\
\hline $55 \% b$ & 0.673267 & 0.732673 & 0.772277 & 0.792079 & 0.80198 & 0.811881 & 0.811881 & 0.831683 & 0.831683 \\
\hline $55 \% \mathrm{t}$ & 643564 & 0.673267 & 0.683168 & 0.693069 & 0.732673 & 0.742574 & 0.772277 & 0.772277 & 0.772277 \\
\hline $60 \% b$ & 0.616162 & 0.686869 & 0.757576 & 0.757576 & 0.79798 & 0.818182 & 0.818182 & 0.848485 & 0.848485 \\
\hline $60 \% t$ & 0.616162 & 0.666667 & 0.686869 & 0.717172 & 0.727273 & 0.737374 & 0.757576 & 0.787879 & 0.808081 \\
\hline $65 \% b$ & 0.678571 & 0.690476 & 0.714286 & 0.72619 & 0.761905 & 0.77381 & 0.785714 & 0.785714 & 0.797619 \\
\hline $65 \% \mathrm{t}$ & 0.583333 & 0.630952 & 0.642857 & 0.678571 & 0.702381 & 0.702381 & 0.761905 & 0.785714 & 0.797619 \\
\hline $70 \% b$ & 0.62069 & 0.678161 & 0.678161 & 0.712644 & 0.712644 & 0.724138 & 0.735632 & 0.747126 & 0.747126 \\
\hline $70 \% t$ & 0.482759 & 0.54023 & 0.597701 & 0.632184 & 0.655172 & 0.666667 & 0.701149 & 0.735632 & 0.747126 \\
\hline $75 \% b$ & 0.671053 & 0.710526 & 0.75 & 0.776316 & 0.815789 & 0.828947 & 0.855263 & 0.868421 & 0.881579 \\
\hline $75 \% t$ & 78947 & 0.657895 & 0.710526 & 0.763158 & 0.789474 & 0.789474 & 0.789474 & 0.802632 & 0.802632 \\
\hline $80 \% b$ & 0.573333 & 0.6 & 0.613333 & 0.613333 & 0.64 & 0.653333 & 0.68 & 0.693333 & 0.693333 \\
\hline $80 \% t$ & 0.426667 & 0.44 & 0.48 & 0.493333 & 0.56 & 0.573333 & 0.586667 & 0.586667 & 0.613333 \\
\hline $85 \% b$ & 0.619718 & 0.661972 & 0.690141 & 0.704225 & 0.71831 & 0.71831 & 0.732394 & 0.732394 & 0.746479 \\
\hline $85 \% \mathrm{t}$ & 0.408451 & 0.478873 & 0.535211 & 0.56338 & 0.591549 & 0.605634 & 0.605634 & 0.605634 & 0.605634 \\
\hline $90 \% b$ & 0.576271 & 0.627119 & 0.661017 & 0.661017 & 0.661017 & 0.661017 & 0.661017 & 0.661017 & 0.661017 \\
\hline $90 \% \mathrm{t}$ & 0.457627 & 0.508475 & 0.508475 & 0.542373 & 0.542373 & 0.542373 & 0.59322 & 0.59322 & 0.610169 \\
\hline $95 \% b$ & 0.803279 & 0.819672 & 0.852459 & 0.852459 & 0.852459 & 0.852459 & 0.852459 & 0.852459 & 0.868852 \\
\hline $95 \% t$ & 0.409836 & 0.491803 & 0.52459 & 0.57377 & 0.622951 & 0.655738 & 0.655738 & 0.672131 & 0.672131 \\
\hline $100 \% b$ & 0.770833 & 0.8125 & 0.833333 & 0.833333 & 0.833333 & 0.833333 & 0.833333 & 0.833333 & 0.833333 \\
\hline
\end{tabular}


Table 20: Cumulative Match Curve For Intra-Trial Randomization on Nose Width Modifications for LBPH

\begin{tabular}{|c|c|c|c|c|c|c|c|c|c|}
\hline trial & R1 Rate & R2 Rate & R3 Rate & R4 Rate & R5 Rate & R6 Rate & R7 Rate & R8 Rate & R9 Rate \\
\hline$-20 \% b$ & 0.731707 & 0.747967 & 0.756098 & 0.756098 & 0.764228 & 0.772358 & 0.780488 & 0.788618 & 0.813008 \\
\hline$-20 \% t$ & 0.756098 & 0.772358 & 0.780488 & 0.780488 & 0.804878 & 0.804878 & 0.813008 & 0.813008 & 0.829268 \\
\hline$-15 \% b$ & 0.671875 & 0.695312 & 0.742188 & 0.757812 & 0.765625 & 0.773438 & 0.78125 & 0.789062 & 0.796875 \\
\hline$-15 \% t$ & 0.695312 & 0.75 & 0.765625 & 0.773438 & 0.789062 & 0.8125 & 0.820312 & 0.820312 & 0.828125 \\
\hline$-10 \% b$ & 0.714286 & 0.759398 & 0.774436 & 0.81203 & 0.827068 & 0.827068 & 0.834586 & 0.834586 & 0.842105 \\
\hline$-10 \% t$ & 0.699248 & 0.75188 & 0.774436 & 0.774436 & 0.796992 & 0.81203 & 0.827068 & 0.827068 & 0.834586 \\
\hline$-5 \% b$ & 0.676692 & 0.729323 & 0.729323 & 0.75188 & 0.759398 & 0.759398 & 0.766917 & 0.774436 & 0.796992 \\
\hline$-5 \% \mathrm{t}$ & 0.676692 & 0.721805 & 0.721805 & 0.759398 & 0.774436 & 0.774436 & 0.774436 & 0.781955 & 0.796992 \\
\hline $5 \%$ & 0.58871 & 0.653226 & 0.66129 & 0.669355 & 0.685484 & 0.685484 & 0.693548 & 0.701613 & 0.709677 \\
\hline $5 \% \mathrm{t}$ & 0.580645 & 0.637097 & 0.645161 & 0.669355 & 0.669355 & 0.677419 & 0.677419 & 0.685484 & 0.701613 \\
\hline $10 \% \mathrm{~b}$ & 0.608 & 0.632 & 0.656 & 0.672 & 0.672 & 0.704 & 0.712 & 0.72 & 0.72 \\
\hline $10 \% \mathrm{t}$ & 0.608 & 0.648 & 0.656 & 0.688 & 0.696 & 0.696 & 0.712 & 0.728 & 0.728 \\
\hline $15 \% b$ & 0.65625 & 0.6875 & 0.71875 & 0.734375 & 0.75 & 0.75 & 0.75 & 0.75 & 0.765625 \\
\hline $15 \% \mathrm{t}$ & 0.625 & 0.679688 & 0.703125 & 0.710938 & 0.71875 & 0.734375 & 0.742188 & 0.773438 & 0.78125 \\
\hline $20 \% b$ & 0.725806 & 0.741935 & 0.806452 & 0.830645 & 0.846774 & 0.870968 & 0.870968 & 0.879032 & 0.927419 \\
\hline $20 \% \mathrm{t}$ & 0.701613 & 0.741935 & 0.766129 & 0.822581 & 0.830645 & 0.846774 & 0.870968 & 0.879032 & 0.903226 \\
\hline
\end{tabular}

Table 21: Cumulative Match Curve For Intra-Trial Randomization on Nose Length Modifications for LBPH

\begin{tabular}{|l|l|l|l|l|l|l|l|l|l|}
\hline trial & R1 Rate & R2 Rate & R3 Rate & R4 Rate & R5 Rate & R6 Rate & R7 Rate & R8 Rate & R9 Rate \\
\hline $\mathbf{- 1 0 0 \% b}$ & 0.734375 & 0.765625 & 0.773438 & 0.773438 & 0.789062 & 0.796875 & 0.796875 & 0.796875 & 0.804688 \\
\hline $\mathbf{- 1 0 0 \% t}$ & 0.734375 & 0.773438 & 0.78125 & 0.78125 & 0.789062 & 0.789062 & 0.796875 & 0.796875 & 0.8125 \\
\hline$-\mathbf{- 9 5} \% \mathbf{b}$ & 0.726562 & 0.765625 & 0.773438 & 0.773438 & 0.789062 & 0.796875 & 0.796875 & 0.796875 & 0.804688 \\
\hline
\end{tabular}




\begin{tabular}{|c|c|c|c|c|c|c|c|c|c|}
\hline$-95 \% t$ & 0.710938 & 0.765625 & 0.773438 & 0.773438 & 0.78125 & 0.789062 & 0.796875 & 0.796875 & 0.804688 \\
\hline$-90 \% b$ & 0.736 & 0.768 & 0.776 & 0.776 & 0.792 & 0.8 & 0.8 & 0.8 & 0.808 \\
\hline$-90 \% t$ & 0.712 & 0.752 & 0.768 & 0.784 & 0.792 & 0.792 & 0.8 & 0.808 & 0.816 \\
\hline$-85 \% b$ & 0.728 & 0.76 & 0.768 & 0.768 & 0.784 & 0.792 & 0.792 & 0.792 & 0.8 \\
\hline$-85 \% t$ & 0.704 & 0.752 & 0.76 & 0.768 & 0.776 & 0.776 & 0.784 & 0.792 & 0.792 \\
\hline$-80 \% b$ & 0.725806 & 0.758065 & 0.766129 & 0.766129 & 0.782258 & 0.790323 & 0.790323 & 0.790323 & 0.798387 \\
\hline$-80 \% t$ & 0.701613 & 0.75 & 0.766129 & 0.766129 & 0.766129 & 0.766129 & 0.774194 & 0.774194 & 0.782258 \\
\hline$-75 \% b$ & 0.728 & 0.76 & 0.768 & 0.768 & 0.784 & 0.792 & 0.792 & 0.792 & 0.8 \\
\hline$-75 \% t$ & 0.664 & 0.728 & 0.76 & 0.76 & 0.76 & 0.76 & 0.784 & 0.784 & 0.792 \\
\hline$-70 \% b$ & 0.730159 & 0.769841 & 0.777778 & 0.777778 & 0.793651 & 0.801587 & 0.801587 & 0.801587 & 0.809524 \\
\hline$-70 \% t$ & 0.698413 & 0.761905 & 0.785714 & 0.793651 & 0.793651 & 0.801587 & 0.809524 & 0.81746 & 0.833333 \\
\hline$-65 \% b$ & 0.728 & 0.768 & 0.776 & 0.776 & 0.792 & 0.8 & 0.8 & 0.8 & 0.808 \\
\hline$-65 \% t$ & 0.672 & 0.768 & 0.792 & 0.792 & 0.8 & 0.808 & 0.824 & 0.84 & 0.84 \\
\hline$-60 \% b$ & 0.730159 & 0.769841 & 0.777778 & 0.777778 & 0.793651 & 0.801587 & 0.801587 & 0.801587 & 0.809524 \\
\hline$-60 \% t$ & 0.706349 & 0.746032 & 0.777778 & 0.785714 & 0.785714 & 0.785714 & 0.801587 & 0.81746 & 0.825397 \\
\hline$-55 \% b$ & 0.733871 & 0.766129 & 0.774194 & 0.774194 & 0.790323 & 0.798387 & 0.798387 & 0.798387 & 0.806452 \\
\hline$-55 \% \mathrm{t}$ & 0.766129 & 0.782258 & 0.790323 & 0.790323 & 0.790323 & 0.790323 & 0.790323 & 0.798387 & 0.806452 \\
\hline$-50 \% b$ & 0.728 & 0.76 & 0.768 & 0.768 & 0.784 & 0.792 & 0.792 & 0.792 & 0.8 \\
\hline$-50 \% t$ & 0.744 & 0.768 & 0.776 & 0.776 & 0.776 & 0.776 & 0.776 & 0.776 & 0.776 \\
\hline$-45 \% b$ & 0.728 & 0.76 & 0.768 & 0.768 & 0.784 & 0.792 & 0.792 & 0.792 & 0.8 \\
\hline$-45 \% t$ & 0.704 & 0.76 & 0.776 & 0.784 & 0.784 & 0.792 & 0.792 & 0.8 & 0.8 \\
\hline$-40 \% b$ & 0.722222 & 0.753968 & 0.761905 & 0.761905 & 0.777778 & 0.785714 & 0.785714 & 0.785714 & 0.793651 \\
\hline$-40 \% t$ & 0.690476 & 0.738095 & 0.753968 & 0.753968 & 0.761905 & 0.769841 & 0.769841 & 0.769841 & 0.777778 \\
\hline$-35 \% b$ & 0.724409 & 0.755906 & 0.76378 & 0.76378 & 0.779528 & 0.787402 & 0.787402 & 0.787402 & 0.795276 \\
\hline$-35 \% t$ & 0.692913 & 0.740157 & 0.755906 & 0.771654 & 0.779528 & 0.795276 & 0.795276 & 0.795276 & 0.795276 \\
\hline$-30 \% b$ & 0.724409 & 0.755906 & 0.76378 & 0.76378 & 0.779528 & 0.787402 & 0.787402 & 0.787402 & 0.795276 \\
\hline
\end{tabular}




\begin{tabular}{|c|c|c|c|c|c|c|c|c|c|}
\hline$-30 \% t$ & 0.708661 & 0.748031 & 0.771654 & 0.771654 & 0.795276 & 0.80315 & 0.80315 & 0.80315 & 0.811024 \\
\hline$-25 \% b$ & 0.710938 & 0.742188 & 0.75 & 0.75 & 0.765625 & 0.773438 & 0.773438 & 0.773438 & 0.78125 \\
\hline$-25 \% \mathrm{t}$ & 703125 & 0.75 & 0.765625 & 0.765625 & 0.78125 & 0.796875 & 0.796875 & 0.804688 & 0.8125 \\
\hline$-20 \% b$ & 0.705426 & 0.744186 & 0.751938 & 0.751938 & 0.767442 & 0.775194 & 0.775194 & 0.775194 & 0.782946 \\
\hline$-20 \% t$ & 0.697674 & 0.767442 & 0.782946 & 0.782946 & 0.790698 & 0.79845 & 0.79845 & 0.79845 & 0.806202 \\
\hline$-15 \% b$ & 0.710938 & 0.75 & 0.757812 & 0.757812 & 0.773438 & 0.78125 & 0.78125 & 0.78125 & 0.789062 \\
\hline$-15 \% t$ & 0.6875 & 0.757812 & 0.765625 & 0.765625 & 0.773438 & 0.773438 & 0.789062 & 0.789062 & 0.796875 \\
\hline$-10 \% b$ & 0.705426 & 0.744186 & 0.751938 & 0.751938 & 0.767442 & 0.775194 & 0.775194 & 0.775194 & 0.782946 \\
\hline$-10 \% t$ & 0.682171 & 0.744186 & 0.75969 & 0.75969 & 0.767442 & 0.775194 & 0.790698 & 0.790698 & 0.79845 \\
\hline$-5 \% b$ & 0.708661 & 0.748031 & 0.755906 & 0.755906 & 0.771654 & 0.779528 & 0.779528 & 0.779528 & 0.787402 \\
\hline$-5 \% t$ & 0.708661 & 0.76378 & 0.771654 & 0.771654 & 0.779528 & 0.779528 & 0.795276 & 0.795276 & 0.80315 \\
\hline $5 \% b$ & 0.736434 & 0.767442 & 0.775194 & 0.775194 & 0.790698 & 0.79845 & 0.79845 & 0.79845 & 0.806202 \\
\hline $5 \% \mathrm{t}$ & 36434 & 0.775194 & 0.775194 & 0.782946 & 0.782946 & 0.782946 & 0.790698 & 0.790698 & 0.79845 \\
\hline $10 \% \mathrm{~b}$ & 0.734375 & 0.765625 & 0.773438 & 0.773438 & 0.789062 & 0.796875 & 0.796875 & 0.796875 & 0.804688 \\
\hline $10 \% \mathrm{t}$ & 0.726562 & 0.773438 & 0.773438 & 0.773438 & 0.773438 & 0.773438 & 0.789062 & 0.789062 & 0.796875 \\
\hline $15 \% b$ & 0.737705 & 0.770492 & 0.778689 & 0.778689 & 0.795082 & 0.803279 & 0.803279 & 0.803279 & 0.811475 \\
\hline $15 \% t$ & 0.737705 & 0.762295 & 0.778689 & 0.778689 & 0.786885 & 0.786885 & 0.795082 & 0.795082 & 0.803279 \\
\hline $20 \% b$ & 0.735043 & 0.752137 & 0.760684 & 0.760684 & 0.777778 & 0.786325 & 0.786325 & 0.786325 & 0.794872 \\
\hline $20 \% \mathrm{t}$ & 0.726496 & 0.752137 & 0.752137 & 0.760684 & 0.769231 & 0.769231 & 0.777778 & 0.794872 & 0.794872 \\
\hline $25 \% b$ & 72807 & 0.754386 & 0.763158 & 0.763158 & 0.77193 & 0.780702 & 0.780702 & 0.780702 & 0.789474 \\
\hline $25 \% \mathrm{t}$ & 0.684211 & 0.736842 & 0.736842 & 0.745614 & 0.754386 & 0.754386 & 0.754386 & 0.754386 & 0.754386 \\
\hline $30 \% b$ & 0.743363 & 0.778761 & 0.787611 & 0.787611 & 0.80531 & 0.814159 & 0.814159 & 0.814159 & 0.823009 \\
\hline $30 \% \mathrm{t}$ & 0.681416 & 0.734513 & 0.743363 & 0.752212 & 0.761062 & 0.769912 & 0.778761 & 0.778761 & 0.787611 \\
\hline $35 \% b$ & 0.738739 & 0.756757 & 0.765766 & 0.765766 & 0.783784 & 0.792793 & 0.792793 & 0.792793 & 0.801802 \\
\hline $35 \% t$ & 0.684685 & 0.711712 & 0.72973 & 0.747748 & 0.756757 & 0.765766 & 0.765766 & 0.774775 & 0.774775 \\
\hline $40 \% b$ & 0.726415 & 0.754717 & 0.764151 & 0.764151 & 0.773585 & 0.783019 & 0.783019 & 0.783019 & 0.792453 \\
\hline
\end{tabular}




\begin{tabular}{|c|c|c|c|c|c|c|c|c|c|}
\hline $40 \% t$ & 0.726415 & 0.754717 & 0.764151 & 0.764151 & 0.792453 & 0.792453 & 0.801887 & 0.801887 & 0.801887 \\
\hline$\overline{45 \% b}$ & 0.72549 & 0.764706 & 0.77451 & 0.77451 & 0.794118 & 0.803922 & 0.803922 & 0.803922 & 0.813725 \\
\hline $45 \% \mathrm{t}$ & 0.696078 & 0.72549 & 0.745098 & 0.754902 & 0.764706 & 0.764706 & 0.764706 & 0.764706 & 0.77451 \\
\hline $50 \% b$ & 0.745098 & 0.77451 & 0.784314 & 0.784314 & 0.803922 & 0.813725 & 0.813725 & 0.813725 & 0.823529 \\
\hline $50 \% t$ & 0.656863 & 0.676471 & 0.715686 & 0.745098 & 0.764706 & 0.764706 & 0.764706 & 0.803922 & 0.803922 \\
\hline $55 \% b$ & 0.734694 & 0.765306 & 0.77551 & 0.77551 & 0.795918 & 0.806122 & 0.806122 & 0.806122 & 0.816327 \\
\hline $55 \% \mathrm{t}$ & 0.653061 & 0.683673 & 0.683673 & 0.693878 & 0.693878 & 0.714286 & 0.744898 & 0.744898 & 0.755102 \\
\hline $60 \% b$ & 0.736842 & 0.778947 & 0.789474 & 0.789474 & 0.8 & 0.810526 & 0.810526 & 0.810526 & 0.821053 \\
\hline $60 \% t$ & 0.631579 & 0.673684 & 0.684211 & 0.684211 & 0.694737 & 0.705263 & 0.715789 & 0.757895 & 0.768421 \\
\hline $65 \% b$ & 0.717391 & 0.76087 & 0.771739 & 0.771739 & 0.782609 & 0.793478 & 0.793478 & 0.793478 & 0.804348 \\
\hline $65 \% \mathrm{t}$ & 0.619565 & 0.652174 & 0.673913 & 0.695652 & 0.717391 & 0.728261 & 0.73913 & 0.76087 & 0.771739 \\
\hline $70 \% b$ & 0.733333 & 0.766667 & 0.777778 & 0.777778 & 0.8 & 0.811111 & 0.811111 & 0.811111 & 0.822222 \\
\hline $70 \% t$ & 0.6 & 0.644444 & 0.666667 & 0.688889 & 0.711111 & 0.722222 & 0.722222 & 0.722222 & 0.722222 \\
\hline $75 \% b$ & 0.686747 & 0.73494 & 0.746988 & 0.746988 & 0.771084 & 0.783133 & 0.783133 & 0.783133 & 0.795181 \\
\hline $75 \% t$ & 0.566265 & 0.626506 & 0.662651 & 0.686747 & 0.698795 & 0.698795 & 0.698795 & 0.698795 & 0.722892 \\
\hline $80 \% b$ & 0.693333 & 0.746667 & 0.76 & 0.76 & 0.773333 & 0.786667 & 0.786667 & 0.786667 & 0.8 \\
\hline $80 \% t$ & 0.56 & 0.613333 & 0.626667 & 0.64 & 0.64 & 0.653333 & 0.666667 & 0.693333 & 0.693333 \\
\hline $85 \% b$ & 0.661972 & 0.71831 & 0.732394 & 0.732394 & 0.746479 & 0.760563 & 0.760563 & 0.760563 & 0.774648 \\
\hline $85 \% t$ & 0.521127 & 0.591549 & 0.619718 & 0.647887 & 0.676056 & 0.676056 & 0.704225 & 0.704225 & 0.704225 \\
\hline $90 \% b$ & 0.6 & 0.666667 & 0.683333 & 0.683333 & 0.7 & 0.716667 & 0.716667 & 0.716667 & 0.733333 \\
\hline $90 \% \mathrm{t}$ & 0.5 & 0.55 & 0.566667 & 0.583333 & 0.583333 & 0.583333 & 0.6 & 0.633333 & 0.633333 \\
\hline $95 \% b$ & 0.68 & 0.72 & 0.74 & 0.74 & 0.76 & 0.76 & 0.76 & 0.76 & 0.78 \\
\hline $95 \% \mathrm{t}$ & 0.46 & 0.54 & 0.58 & 0.62 & 0.64 & 0.68 & 0.68 & 0.68 & 0.68 \\
\hline $100 \% b$ & 0.659574 & 0.702128 & 0.723404 & 0.723404 & 0.744681 & 0.765957 & 0.765957 & 0.765957 & 0.787234 \\
\hline $100 \% t$ & 0.531915 & 0.553191 & 0.617021 & 0.617021 & 0.638298 & 0.680851 & 0.680851 & 0.702128 & 0.702128 \\
\hline
\end{tabular}




\begin{tabular}{|c|c|c|c|c|c|c|c|c|c|}
\hline trial & R1 Rate & R2 Rate & R3 Rate & R4 Rate & R5 Rate & R6 Rate & R7 Rate & R8 Rate & R9 Rate \\
\hline$-20 \% b$ & 0.741935 & 0.782258 & 0.790323 & 0.790323 & 0.806452 & 0.814516 & 0.814516 & 0.814516 & 0.822581 \\
\hline$-20 \% t$ & 0.725806 & 0.798387 & 0.798387 & 0.814516 & 0.814516 & 0.814516 & 0.814516 & 0.822581 & 0.822581 \\
\hline$-15 \% b$ & 0.730159 & 0.761905 & 0.769841 & 0.769841 & 0.785714 & 0.793651 & 0.793651 & 0.793651 & 0.801587 \\
\hline$-15 \% t$ & 0.730159 & 0.785714 & 0.785714 & 0.785714 & 0.785714 & 0.785714 & 0.793651 & 0.801587 & 0.801587 \\
\hline$-10 \% b$ & 0.717557 & 0.748092 & 0.755725 & 0.755725 & 0.770992 & 0.778626 & 0.778626 & 0.778626 & 0.78626 \\
\hline$-10 \% t$ & 0.717557 & 0.755725 & 0.770992 & 0.770992 & 0.770992 & 0.770992 & 0.770992 & 0.770992 & 0.770992 \\
\hline$-5 \% b$ & 0.714286 & 0.744361 & 0.75188 & 0.75188 & 0.766917 & 0.774436 & 0.774436 & 0.774436 & 0.781955 \\
\hline$-5 \% \mathrm{t}$ & 0.729323 & 0.759398 & 0.766917 & 0.766917 & 0.766917 & 0.766917 & 0.766917 & 0.766917 & 0.774436 \\
\hline $5 \% b$ & 0.708661 & 0.748031 & 0.755906 & 0.755906 & 0.771654 & 0.779528 & 0.779528 & 0.779528 & 0.787402 \\
\hline $5 \% t$ & 0.700787 & 0.732283 & 0.748031 & 0.748031 & 0.779528 & 0.795276 & 0.80315 & 0.80315 & 0.80315 \\
\hline $10 \% b$ & 0.714286 & 0.753968 & 0.761905 & 0.761905 & 0.777778 & 0.785714 & 0.785714 & 0.785714 & 0.793651 \\
\hline $10 \% \mathrm{t}$ & 0.706349 & 0.730159 & 0.753968 & 0.753968 & 0.777778 & 0.793651 & 0.793651 & 0.793651 & 0.793651 \\
\hline $15 \% b$ & 0.709677 & 0.75 & 0.758065 & 0.758065 & 0.774194 & 0.782258 & 0.782258 & 0.782258 & 0.790323 \\
\hline $15 \% \mathrm{t}$ & 0.677419 & 0.733871 & 0.741935 & 0.75 & 0.766129 & 0.790323 & 0.798387 & 0.798387 & 0.798387 \\
\hline $20 \% \mathrm{~b}$ & 0.721311 & 0.762295 & 0.770492 & 0.770492 & 0.786885 & 0.795082 & 0.795082 & 0.795082 & 0.803279 \\
\hline $20 \% t$ & 0.704918 & 0.721311 & 0.745902 & 0.762295 & 0.770492 & 0.778689 & 0.795082 & 0.795082 & 0.795082 \\
\hline
\end{tabular}

Table 23: Cumulative Match Curve For Inter-Trial Randomization on Nose Length Modifications for LBPH 


\section{APPENDIX C : IMAGE SIMILARITY VS. RANK-1 RECOGNITION RATES}

As Section 4.3 discusses, image similarity compares two image's pixels as a "signal" which can be objectively compared with Peak Signal-to-Noise Ratio (in dB). Given the way PSNR is calculated, there is no upper limit on similarity between images, but only has a lower limit ( $0 \mathrm{~dB})$. In order to determine the local limit for an image, Case $\mathrm{A}$ is used by comparing the original (unmodified image) with the same image, which is compared against the PSNR from comparing the original image and the modified image. For scope, Case B is just the PSNR from the comparing the original image and the modified image. For both Case A and B, the average and median for all image pairs used in the test image set are calculated. In addition, the Rank-1 Recognition Rate (R1 Rate) has two values for each trial, a baseline and treatment rate. The baseline rate is the recognition rate for the unmodified test images, and the treatment is the parallel test set of modified images.

\begin{tabular}{|c|c|c|c|c|c|c|}
\hline Modification & $\begin{array}{c}\text { PSNR(Avg- } \\
\text { CaseA) }\end{array}$ & $\begin{array}{c}\text { PSNR(Avg- } \\
\text { CaseB) }\end{array}$ & $\begin{array}{c}\text { PSNR(Median- } \\
\text { CaseA) }\end{array}$ & $\begin{array}{c}\text { PSNR(Median- } \\
\text { CaseB) }\end{array}$ & $\begin{array}{c}\text { R1 Rate } \\
\text { (Baseline) }\end{array}$ & $\begin{array}{c}\text { R1 Rate } \\
\text { (Treatment) }\end{array}$ \\
\hline $\mathbf{- 1 0 0 \%}$ & 336.587608 & 24.614392 & 335.993380 & 24.614392 & 0.539683 & 0.579365 \\
\hline $\mathbf{- 9 5} \%$ & 336.243670 & 24.958329 & 335.998649 & 24.958329 & 0.653226 & 0.645161 \\
\hline $\mathbf{- 9 0} \%$ & 335.776561 & 25.425438 & 335.551137 & 25.425438 & 0.624000 & 0.608000 \\
\hline $\mathbf{- 8 5} \%$ & 336.528340 & 24.673659 & 336.492519 & 24.673659 & 0.598361 & 0.557377 \\
\hline $\mathbf{- 8 0} \%$ & 335.837114 & 25.364885 & 336.029389 & 25.364885 & 0.624000 & 0.656000 \\
\hline $\mathbf{- 7 5} \%$ & 336.423378 & 24.778621 & 336.613343 & 24.778621 & 0.491667 & 0.466667 \\
\hline $\mathbf{- 7 0} \%$ & 335.945671 & 25.256328 & 335.906654 & 25.256328 & 0.608333 & 0.666667 \\
\hline $\mathbf{- 6 5} \%$ & 335.769655 & 25.432344 & 335.888853 & 25.432344 & 0.572581 & 0.604839 \\
\hline $\mathbf{- 6 0} \%$ & 336.431079 & 24.770920 & 335.844956 & 24.770920 & 0.588710 & 0.572581 \\
\hline $\mathbf{- 5 5} \%$ & 336.420048 & 24.781951 & 336.469136 & 24.781951 & 0.619835 & 0.619835 \\
\hline $\mathbf{- 5 0} \%$ & 335.660673 & 25.541326 & 335.776811 & 25.541326 & 0.491935 & 0.516129 \\
\hline $\mathbf{4 5} \%$ & 335.827438 & 25.374561 & 335.705344 & 25.374561 & 0.523077 & 0.515385 \\
\hline $\mathbf{4 0} \%$ & 335.158643 & 26.043356 & 335.070611 & 26.043356 & 0.551181 & 0.543307 \\
\hline $\mathbf{- 3 5} \%$ & 335.156914 & 26.045085 & 336.059280 & 26.045085 & 0.587302 & 0.571429 \\
\hline $\mathbf{- 3 0} \%$ & 335.113130 & 26.088869 & 335.661135 & 26.088869 & 0.562500 & 0.539063 \\
\hline $\mathbf{- 2 5} \%$ & 334.812291 & 26.389709 & 335.067070 & 26.389709 & 0.587302 & 0.619048 \\
\hline $\mathbf{- 2 0} \%$ & 332.959888 & 28.242111 & 334.471691 & 28.242111 & 0.587786 & 0.618321 \\
\hline $\mathbf{- 1 5} \%$ & 333.012594 & 28.189405 & 334.170803 & 28.189405 & 0.608000 & 0.624000 \\
\hline $\mathbf{- 1 0} \%$ & 331.266850 & 29.935149 & 333.194351 & 29.935149 & 0.562500 & 0.578125 \\
\hline $\mathbf{- 5} \%$ & 331.339998 & 29.862001 & 333.717836 & 29.862001 & 0.692913 & 0.653543 \\
\hline & & & & & \\
\hline
\end{tabular}




\begin{tabular}{|c|c|c|c|c|c|c|}
\hline $5 \%$ & 322.508806 & 38.693193 & 333.217086 & 38.693193 & 0.528000 & 0.552000 \\
\hline $10 \%$ & 333.367704 & 27.834295 & 335.094547 & 27.834295 & 0.519380 & 0.488372 \\
\hline $15 \%$ & 334.762534 & 26.439466 & 335.287570 & 26.439466 & 0.533898 & 0.550847 \\
\hline $20 \%$ & 336.156946 & 25.045053 & 336.274106 & 25.045053 & 0.504202 & 0.512605 \\
\hline $25 \%$ & 336.640717 & 24.561282 & 336.532693 & 24.561282 & 0.564103 & 0.521368 \\
\hline $30 \%$ & 337.148244 & 24.053755 & 336.966701 & 24.053755 & 0.559633 & 0.577982 \\
\hline $35 \%$ & 338.177357 & 23.024642 & 337.566633 & 23.024642 & 0.478992 & 0.445378 \\
\hline $40 \%$ & 337.932744 & 23.269255 & 337.759722 & 23.269255 & 0.609524 & 0.561905 \\
\hline $45 \%$ & 338.907437 & 22.294562 & 338.327956 & 22.294562 & 0.529412 & 0.509804 \\
\hline$\overline{50 \%}$ & 339.367592 & 21.834407 & 339.200682 & 21.834407 & 0.613208 & 0.575472 \\
\hline $55 \%$ & 339.069055 & 22.132944 & 338.724809 & 22.132944 & 0.551020 & 0.479592 \\
\hline $60 \%$ & 339.470424 & 21.731575 & 339.622816 & 21.731575 & 0.630000 & 0.510000 \\
\hline $65 \%$ & 339.954370 & 21.247629 & 339.181573 & 21.247629 & 0.483516 & 0.329670 \\
\hline $70 \%$ & 340.182083 & 21.019916 & 339.710681 & 21.019916 & 0.528090 & 0.438202 \\
\hline $75 \%$ & 340.846027 & 20.355972 & 340.712220 & 20.355972 & 0.653846 & 0.474359 \\
\hline $80 \%$ & 341.643421 & 19.558578 & 341.006738 & 19.558578 & 0.631579 & 0.394737 \\
\hline $85 \%$ & 341.469909 & 19.732091 & 340.840963 & 19.732091 & 0.571429 & 0.414286 \\
\hline $90 \%$ & 342.223368 & 18.978631 & 341.875599 & 18.978631 & 0.612903 & 0.354839 \\
\hline $95 \%$ & 342.551456 & 18.650543 & 341.828882 & 18.650543 & 0.532258 & 0.387097 \\
\hline $100 \%$ & 342.705104 & 18.496895 & 341.986013 & 18.496895 & 0.673913 & 0.456522 \\
\hline
\end{tabular}

Table 24: LDA Image Similarity (PSNR) vs. Rank-1 Recognition Rates for Intra-Trial Randomization on Nose Width Modifications

Table 24 is shows all of the PSNR values (average and median) for both Case A and B LDA IntraTrial Randomization. In Table 24, Figure 16 is the graph of the averages and medians of Case A PSNR plotted against the Rank-1 Recognition Rates for all Width Modifications. Figure 17 is the graph of Case B.

\begin{tabular}{|c|c|c|c|c|c|c|}
\hline Modification & $\begin{array}{c}\text { PSNR(Avg- } \\
\text { CaseA) }\end{array}$ & $\begin{array}{c}\text { PSNR(Avg- } \\
\text { CaseB) }\end{array}$ & $\begin{array}{c}\text { PSNR(Median- } \\
\text { CaseA) }\end{array}$ & $\begin{array}{c}\text { PSNR(Median- } \\
\text { CaseB) }\end{array}$ & $\begin{array}{c}\text { R1 Rate } \\
\text { (Baseline) }\end{array}$ & $\begin{array}{c}\text { R1 Rate } \\
\text { (Treatment) }\end{array}$ \\
\hline $\mathbf{- 2 0 \%}$ & 334.355166 & 26.846833 & 335.398494 & 26.846833 & 0.500000 & 0.459016 \\
\hline$-15 \%$ & 328.455743 & 32.746257 & 330.171444 & 32.746257 & 0.610687 & 0.641221 \\
\hline$-10 \%$ & 317.314394 & 43.887605 & 326.904314 & 43.887605 & 0.484615 & 0.492308 \\
\hline
\end{tabular}




\begin{tabular}{|c|c|c|c|c|c|c|}
\hline $\mathbf{- 5} \%$ & 217.610578 & 143.591421 & 309.422606 & 143.591421 & 0.507463 & 0.514925 \\
\hline $\mathbf{5 \%}$ & 334.955883 & 26.246116 & 335.575020 & 26.246116 & 0.632000 & 0.616000 \\
\hline $\mathbf{1 0} \%$ & 333.069189 & 28.132811 & 336.256279 & 28.132811 & 0.542636 & 0.542636 \\
\hline $\mathbf{1 5 \%}$ & 336.438814 & 24.763185 & 336.220576 & 24.763185 & 0.524590 & 0.540984 \\
\hline $\mathbf{2 0} \%$ & 338.174353 & 23.027646 & 338.198558 & 23.027646 & 0.603306 & 0.578512 \\
\hline
\end{tabular}

Table 25: LDA Image Similarity (PSNR) vs. Rank-1 Recognition Rate for Intra-Trial Randomization on Nose Length Modifications

Showing the same breakdown for test images as Table 24, Table 25 shows the image similarity image for the nose length modification trials. Table 25 can be seen in graphical form as Figure 18 for Case B PSNR, LDA values. This table is meant to be an addition for Section 4.3.

\begin{tabular}{|c|c|c|c|c|c|c|}
\hline Modification & $\begin{array}{c}\text { PSNR(Avg- } \\
\text { CaseA) }\end{array}$ & $\begin{array}{c}\text { PSNR(Avg- } \\
\text { CaseB) }\end{array}$ & $\begin{array}{c}\text { PSNR(Median- } \\
\text { CaseA) }\end{array}$ & $\begin{array}{c}\text { PSNR(Median- } \\
\text { CaseB) }\end{array}$ & $\begin{array}{c}\text { R1 Rate } \\
\text { (Baseline) }\end{array}$ & $\begin{array}{c}\text { R1 Rate } \\
\text { (Treatment) }\end{array}$ \\
\hline $\mathbf{- 1 0 0 \%}$ & 336.437413 & 24.764586 & 336.208631 & 24.764586 & 0.632813 & 0.648438 \\
\hline $\mathbf{- 9 5} \%$ & 336.308018 & 24.893981 & 336.258038 & 24.893981 & 0.632813 & 0.648438 \\
\hline $\mathbf{- 9 0} \%$ & 335.992412 & 25.209587 & 335.584422 & 25.209587 & 0.632000 & 0.656000 \\
\hline $\mathbf{- 8 5} \%$ & 336.649904 & 24.552095 & 336.903301 & 24.552095 & 0.632000 & 0.624000 \\
\hline $\mathbf{- 8 0} \%$ & 336.418780 & 24.783219 & 336.430099 & 24.783219 & 0.629032 & 0.620968 \\
\hline $\mathbf{- 7 5} \%$ & 336.901975 & 24.300024 & 336.828620 & 24.300024 & 0.632000 & 0.640000 \\
\hline $\mathbf{- 7 0} \%$ & 336.171176 & 25.030823 & 335.962090 & 25.030823 & 0.634921 & 0.650794 \\
\hline $\mathbf{- 6 5} \%$ & 335.957536 & 25.244463 & 335.922119 & 25.244463 & 0.640000 & 0.624000 \\
\hline $\mathbf{- 6 0} \%$ & 336.421276 & 24.780723 & 335.819573 & 24.780723 & 0.642857 & 0.619048 \\
\hline $\mathbf{- 5 5} \%$ & 336.445274 & 24.756725 & 336.394887 & 24.756725 & 0.645161 & 0.637097 \\
\hline $\mathbf{5 0} \%$ & 336.205694 & 24.996305 & 335.850745 & 24.996305 & 0.632000 & 0.608000 \\
\hline $\mathbf{4 5} \%$ & 336.009181 & 25.192818 & 335.809539 & 25.192818 & 0.640000 & 0.632000 \\
\hline $\mathbf{4 0} \%$ & 336.207064 & 24.994935 & 336.353902 & 24.994935 & 0.634921 & 0.626984 \\
\hline $\mathbf{- 3 5} \%$ & 335.432531 & 25.769469 & 335.855507 & 25.769469 & 0.637795 & 0.645669 \\
\hline $\mathbf{- 3 0} \%$ & 335.606619 & 25.595380 & 335.927912 & 25.595380 & 0.637795 & 0.614173 \\
\hline $\mathbf{- 2 5} \%$ & 334.475011 & 26.726988 & 335.081198 & 26.726988 & 0.625000 & 0.625000 \\
\hline $\mathbf{- 2 0} \%$ & 333.721523 & 27.480476 & 334.883881 & 27.480476 & 0.620155 & 0.643411 \\
\hline $\mathbf{- 1 5} \%$ & 332.827551 & 28.374448 & 334.229201 & 28.374448 & 0.625000 & 0.609375 \\
\hline & & & & & \\
\hline
\end{tabular}




\begin{tabular}{|c|c|c|c|c|c|c|}
\hline$-10 \%$ & 331.862326 & 29.339673 & 334.143722 & 29.339673 & 0.620155 & 0.620155 \\
\hline$-5 \%$ & 323.766875 & 37.435124 & 333.895909 & 37.435124 & 0.622047 & 0.629921 \\
\hline $5 \%$ & 325.976416 & 35.225583 & 333.726471 & 35.225583 & 0.635659 & 0.589147 \\
\hline $10 \%$ & 333.123579 & 28.078420 & 333.966487 & 28.078420 & 0.640625 & 0.601563 \\
\hline $15 \%$ & 334.761387 & 26.440612 & 335.328621 & 26.440612 & 0.639344 & 0.598361 \\
\hline $20 \%$ & 335.612708 & 25.589291 & 335.719509 & 25.589291 & 0.623932 & 0.598291 \\
\hline $25 \%$ & 336.434131 & 24.767868 & 336.167733 & 24.767868 & 0.631579 & 0.570175 \\
\hline $30 \%$ & 337.559031 & 23.642969 & 337.319073 & 23.642969 & 0.637168 & 0.575221 \\
\hline $35 \%$ & 337.982363 & 23.219636 & 337.273095 & 23.219636 & 0.630631 & 0.549550 \\
\hline $40 \%$ & 337.880663 & 23.321336 & 337.651415 & 23.321336 & 0.632075 & 0.594340 \\
\hline $45 \%$ & 338.764696 & 22.437303 & 337.913964 & 22.437303 & 0.647059 & 0.519608 \\
\hline $50 \%$ & 339.391511 & 21.810488 & 339.200682 & 21.810488 & 0.637255 & 0.588235 \\
\hline $55 \%$ & 339.245168 & 21.956831 & 338.463058 & 21.956831 & 0.642857 & 0.551020 \\
\hline $60 \%$ & 339.831044 & 21.370955 & 339.927314 & 21.370955 & 0.663158 & 0.547368 \\
\hline $65 \%$ & 340.019656 & 21.182343 & 339.197860 & 21.182343 & 0.663043 & 0.500000 \\
\hline $70 \%$ & 340.513543 & 20.688456 & 340.285381 & 20.688456 & 0.688889 & 0.500000 \\
\hline $75 \%$ & 340.999074 & 20.202925 & 340.838011 & 20.202925 & 0.638554 & 0.518072 \\
\hline $80 \%$ & 341.208700 & 19.993299 & 340.926612 & 19.993299 & 0.653333 & 0.466667 \\
\hline $85 \%$ & 341.410179 & 19.791820 & 341.063875 & 19.791820 & 0.633803 & 0.535211 \\
\hline $90 \%$ & 342.019619 & 19.182380 & 341.798121 & 19.182380 & 0.600000 & 0.450000 \\
\hline $95 \%$ & 342.153272 & 19.048727 & 341.799376 & 19.048727 & 0.660000 & 0.440000 \\
\hline$\overline{100 \%}$ & 342.077372 & 19.124627 & 341.841587 & 19.124627 & 0.680851 & 0.468085 \\
\hline
\end{tabular}

Table 26: LDA Image Similarity vs. Rank-1 Recognition Rate for Inter-

Trial Randomization on Nose Width Modifications

In Table 26, the image similarity results for nose width modifications are shown. Table 26 can be used to visualize Case A and Case B, LDA, Inter-Trial Randomization for nose width modifications.

\begin{tabular}{|c|c|c|c|c|c|c|c|}
\hline Modification & $\begin{array}{c}\text { PSNR(Avg- } \\
\text { CaseA) }\end{array}$ & $\begin{array}{c}\text { PSNR(Avg- } \\
\text { CaseB) }\end{array}$ & $\begin{array}{c}\text { PSNR(Median- } \\
\text { CaseA) }\end{array}$ & $\begin{array}{c}\text { PSNR(Median- } \\
\text { CaseB) }\end{array}$ & $\begin{array}{c}\text { R1 Rate } \\
\text { (Baseline) }\end{array}$ & $\begin{array}{c}\text { Rate } \\
\text { (Treatment) }\end{array}$ \\
\hline $\mathbf{- 2 0} \%$ & 334.751678 & 26.450321 & 335.283759 & 26.450321 & 0.645161 & 0.645161 \\
\hline
\end{tabular}




\begin{tabular}{|c|c|c|c|c|c|c|}
\hline $\mathbf{- 1 5} \%$ & 327.970224 & 33.231775 & 329.853072 & 33.231775 & 0.626984 & 0.619048 \\
\hline $\mathbf{- 1 0} \%$ & 315.350594 & 45.851405 & 327.050782 & 45.851405 & 0.618321 & 0.618321 \\
\hline $\mathbf{- 5} \%$ & 222.524746 & 138.677253 & 307.735052 & 138.677253 & 0.616541 & 0.631579 \\
\hline $\mathbf{5 \%}$ & 335.254793 & 25.947206 & 335.336610 & 25.947206 & 0.622047 & 0.598425 \\
\hline $\mathbf{1 0} \%$ & 335.564236 & 25.637763 & 335.740212 & 25.637763 & 0.626984 & 0.603175 \\
\hline $\mathbf{1 5 \%}$ & 336.498579 & 24.703421 & 336.407225 & 24.703421 & 0.620968 & 0.588710 \\
\hline $\mathbf{2 0} \%$ & 338.222496 & 22.979503 & 338.345672 & 22.979503 & 0.639344 & 0.581967 \\
\hline
\end{tabular}

Table 27: LDA Image Similarity (PSNR) vs. Rank-1 Recognition Rates for Inter-Trial Randomization on Nose Length Modifications

Only differing from Table 27 in the modifications that are shown Table 27 shows only the nose length modification trials. Table 27 is the numerical form of Case A and Case B, LDA, PSNR for nose length modifications. For additional understanding of Table 27, look for Section 4.3 for more details.

Next the PSNR graphs for both PCA and LBPH will be shown in the same order as was shown for LDA. Less explanation will be presented since the conclusions and discussion can be found in Section 4.3, 4.7, and 4.8.

\begin{tabular}{|l|l|l|l|r|r|r|}
\hline Modification & $\begin{array}{c}\text { PSNR(Avg- } \\
\text { CaseA) }\end{array}$ & $\begin{array}{c}\text { PSNR(Avg- } \\
\text { CaseB) }\end{array}$ & $\begin{array}{c}\text { PSNR(Median- } \\
\text { CaseA) }\end{array}$ & $\begin{array}{c}\text { PSNR(Median- } \\
\text { CaseB) }\end{array}$ & $\begin{array}{c}\text { R1 Rate } \\
\text { (Baseline) }\end{array}$ & $\begin{array}{c}\text { R1 Rate } \\
\text { (Treatment) }\end{array}$ \\
\hline $\mathbf{- 1 0 0 \%}$ & 336.241613 & 24.960386 & 335.820915 & 24.960386 & 0.601563 & 0.601563 \\
\hline $\mathbf{- 9 5} \%$ & 335.989569 & 25.212430 & 336.172695 & 25.212430 & 0.582677 & 0.582677 \\
\hline $\mathbf{- 9 0} \%$ & 335.196672 & 26.005327 & 335.311640 & 26.005327 & 0.544000 & 0.560000 \\
\hline $\mathbf{- 8 5} \%$ & 336.669374 & LBPH & 336.714332 & 24.532626 & 0.614754 & 0.590164 \\
\hline $\mathbf{- 8 0} \%$ & 336.200060 & 25.001939 & 336.013393 & 25.001939 & 0.639344 & 0.655738 \\
\hline $\mathbf{- 7 5} \%$ & 336.906504 & 24.295495 & 336.850269 & 24.295495 & 0.590164 & 0.573770 \\
\hline $\mathbf{- 7 0} \%$ & 336.277526 & 24.924473 & 336.065010 & 24.924473 & 0.592000 & 0.576000 \\
\hline $\mathbf{- 6 5} \%$ & 336.169819 & 25.032180 & 336.240020 & 25.032180 & 0.590551 & 0.582677 \\
\hline $\mathbf{- 6 0} \%$ & 336.362116 & 24.839883 & 336.178065 & 24.839883 & 0.595238 & 0.579365 \\
\hline $\mathbf{5 5} \%$ & 336.450187 & 24.751812 & 336.195419 & 24.751812 & 0.551181 & 0.574803 \\
\hline $\mathbf{5 0} \%$ & 336.493707 & 24.708292 & 336.746277 & 24.708292 & 0.559055 & 0.622047 \\
\hline
\end{tabular}




\begin{tabular}{|c|c|c|c|c|c|c|}
\hline$-45 \%$ & 336.103863 & 25.098136 & 336.516924 & 25.098136 & 0.566929 & 0.582677 \\
\hline$-40 \%$ & 336.012958 & 25.189041 & 335.729863 & 25.189041 & 0.563492 & 0.547619 \\
\hline$-35 \%$ & 335.851606 & 25.350393 & 335.828875 & 25.350393 & 0.653226 & 0.637097 \\
\hline$-30 \%$ & 335.240624 & 25.961375 & 335.806438 & 25.961375 & 0.563492 & 0.563492 \\
\hline$-25 \%$ & 334.147058 & 27.054941 & 335.072277 & 27.054941 & 0.616000 & 0.608000 \\
\hline$-20 \%$ & 333.587385 & 27.614614 & 334.621684 & 27.614614 & 0.621212 & 0.674242 \\
\hline$-15 \%$ & 333.483528 & 27.718471 & 334.059332 & 27.718471 & 0.620155 & 0.604651 \\
\hline$-10 \%$ & 331.015905 & 30.186094 & 333.277680 & 30.186094 & 0.522727 & 0.530303 \\
\hline$-5 \%$ & 323.054086 & 38.147913 & 333.634774 & 38.147913 & 0.543307 & 0.535433 \\
\hline $5 \%$ & 321.252228 & 39.949771 & 333.854077 & 39.949771 & 0.607692 & 0.600000 \\
\hline $10 \%$ & 333.098654 & 28.103345 & 333.849099 & 28.103345 & 0.650794 & 0.595238 \\
\hline $15 \%$ & 334.854659 & 26.347340 & 335.604759 & 26.347340 & 0.633333 & 0.633333 \\
\hline $20 \%$ & 335.560174 & 25.641825 & 335.882941 & 25.641825 & 0.521739 & 0.504348 \\
\hline $25 \%$ & 336.696757 & 24.505242 & 336.306677 & 24.505242 & 0.500000 & 0.517544 \\
\hline $30 \%$ & 337.014319 & 24.187681 & 336.575353 & 24.187681 & 0.513761 & 0.532110 \\
\hline $35 \%$ & 337.720292 & 23.481707 & 337.273095 & 23.481707 & 0.575221 & 0.548673 \\
\hline $40 \%$ & 337.629835 & 23.572164 & 337.643774 & 23.572164 & 0.561905 & 0.561905 \\
\hline $45 \%$ & 338.536848 & 22.665151 & 338.026109 & 22.665151 & 0.660550 & 0.587156 \\
\hline $50 \%$ & 339.192493 & 22.009506 & 338.982040 & 22.009506 & 0.562500 & 0.517857 \\
\hline $55 \%$ & 338.877726 & 22.324273 & 338.623351 & 22.324273 & 0.705882 & 0.745098 \\
\hline $60 \%$ & 339.595824 & 21.606175 & 339.692963 & 21.606175 & 0.585106 & 0.563830 \\
\hline $65 \%$ & 339.699009 & 21.502990 & 339.214146 & 21.502990 & 0.517647 & 0.529412 \\
\hline $70 \%$ & 340.638155 & 20.563844 & 340.369242 & 20.563844 & 0.674157 & 0.606742 \\
\hline $75 \%$ & 341.090568 & 20.111431 & 340.679391 & 20.111431 & 0.539474 & 0.513158 \\
\hline $80 \%$ & 341.613807 & 19.588192 & 340.926612 & 19.588192 & 0.574713 & 0.563218 \\
\hline $85 \%$ & 341.399468 & 19.802531 & 340.724287 & 19.802531 & 0.666667 & 0.541667 \\
\hline $90 \%$ & 341.680139 & 19.521861 & 341.566016 & 19.521861 & 0.534483 & 0.517241 \\
\hline $95 \%$ & 342.881262 & 18.320737 & 341.799376 & 18.320737 & 0.538462 & 0.365385 \\
\hline $100 \%$ & 342.583503 & 18.618496 & 341.805065 & 18.618496 & 0.750000 & 0.522727 \\
\hline
\end{tabular}

Table 28: PCA Image Similarity (PSNR) vs. Rank-1 Recognition Rates for Intra-Trial Randomization on Nose Width Modifications 


\begin{tabular}{|l|c|c|c|c|c|c|}
\hline Modification & $\begin{array}{c}\text { PSNR(Avg- } \\
\text { CaseA) }\end{array}$ & $\begin{array}{c}\text { PSNR(Avg- } \\
\text { CaseB) }\end{array}$ & $\begin{array}{c}\text { PSNR(Median- } \\
\text { CaseA) }\end{array}$ & $\begin{array}{c}\text { PSNR(Median- } \\
\text { CaseB) }\end{array}$ & $\begin{array}{c}\text { R1 Rate } \\
\text { (Baseline) }\end{array}$ & $\begin{array}{c}\text { R1 Rate } \\
\text { (Treatment) }\end{array}$ \\
\hline $\mathbf{- 2 0 \%}$ & 334.603126 & 26.598873 & 335.532285 & 26.598873 & 0.549180 & 0.524590 \\
\hline $\mathbf{- 1 5 \%}$ & 328.229080 & 32.972919 & 330.832813 & 32.972919 & 0.589147 & 0.596899 \\
\hline $\mathbf{- 1 0 \%}$ & 317.511705 & 43.690294 & 328.298734 & 43.690294 & 0.601626 & 0.593496 \\
\hline $\mathbf{- 5 \%}$ & 216.828471 & 144.373528 & 307.262902 & 144.373528 & 0.533835 & 0.541353 \\
\hline $\mathbf{5 \%}$ & 335.395720 & 25.806279 & 335.550483 & 25.806279 & 0.593750 & 0.562500 \\
\hline $\mathbf{1 0} \%$ & 333.158282 & 28.043717 & 336.173980 & 28.043717 & 0.542636 & 0.573643 \\
\hline $\mathbf{1 5 \%}$ & 336.392668 & 24.809331 & 336.097774 & 24.809331 & 0.608000 & 0.592000 \\
\hline $\mathbf{2 0 \%}$ & 338.098943 & 23.103056 & 338.479572 & 23.103056 & 0.575000 & 0.600000 \\
\hline
\end{tabular}

Table 29:PCA Image Similarity (PSNR) vs. Rank-1 Recognition Rates for Intra-Trial Randomization on Nose Length Modifications

\begin{tabular}{|c|c|c|c|c|c|c|}
\hline Modification & $\begin{array}{l}\text { PSNR(Avg- } \\
\text { CaseA) }\end{array}$ & $\begin{array}{l}\text { PSNR(Avg- } \\
\text { CaseB) }\end{array}$ & $\begin{array}{l}\text { PSNR(Median- } \\
\text { CaseA) }\end{array}$ & $\begin{array}{l}\text { PSNR(Median- } \\
\text { CaseB) }\end{array}$ & $\begin{array}{l}\text { R1 Rate } \\
\text { (Baseline) }\end{array}$ & $\begin{array}{l}\text { R1 Rate } \\
\text { (Treatment) }\end{array}$ \\
\hline$-100 \%$ & 336.437413 & 24.764586 & 336.208631 & 24.764586 & 0.578125 & 0.609375 \\
\hline$-95 \%$ & 336.308018 & 24.893981 & 336.258038 & 24.893981 & 0.570313 & 0.593750 \\
\hline$-90 \%$ & 335.992412 & 25.209587 & 335.584422 & 25.209587 & 0.584000 & 0.584000 \\
\hline$-85 \%$ & 336.649904 & 24.552095 & 336.903301 & 24.552095 & 0.568000 & 0.576000 \\
\hline$-80 \%$ & 336.418780 & 24.783219 & 336.430099 & 24.783219 & 0.564516 & 0.556452 \\
\hline$-75 \%$ & 336.901975 & 24.300024 & 336.828620 & 24.300024 & 0.568000 & 0.576000 \\
\hline$-70 \%$ & 336.171176 & 25.030823 & 335.962090 & 25.030823 & 0.571429 & 0.587302 \\
\hline$-65 \%$ & 335.957536 & 25.244463 & 335.922119 & 25.244463 & 0.576000 & 0.576000 \\
\hline$-60 \%$ & 336.421276 & 24.780723 & 335.819573 & 24.780723 & 0.579365 & 0.603175 \\
\hline$-55 \%$ & 336.445274 & 24.756725 & 336.394887 & 24.756725 & 0.580645 & 0.620968 \\
\hline$-50 \%$ & 336.205694 & 24.996305 & 335.850745 & 24.996305 & 0.568000 & 0.592000 \\
\hline$-45 \%$ & 336.009181 & 25.192818 & 335.809539 & 25.192818 & 0.576000 & 0.616000 \\
\hline$-40 \%$ & 336.207064 & 24.994935 & 336.353902 & 24.994935 & 0.571429 & 0.579365 \\
\hline$-35 \%$ & 335.432531 & 25.769469 & 335.855507 & 25.769469 & 0.574803 & 0.574803 \\
\hline$-30 \%$ & 335.606619 & 25.595380 & 335.927912 & 25.595380 & 0.574803 & 0.559055 \\
\hline$-25 \%$ & 334.475011 & 26.726988 & 335.081198 & 26.726988 & 0.570313 & 0.554688 \\
\hline$-20 \%$ & 333.721523 & 27.480476 & 334.883881 & 27.480476 & 0.558140 & 0.596899 \\
\hline
\end{tabular}




\begin{tabular}{|c|c|c|c|c|c|c|}
\hline$-15 \%$ & 332.827551 & 28.374448 & 334.229201 & 28.374448 & 0.562500 & 0.585938 \\
\hline$-10 \%$ & 331.862326 & 29.339673 & 334.143722 & 29.339673 & 0.558140 & 0.573643 \\
\hline$-5 \%$ & 323.766875 & 37.435124 & 333.895909 & 37.435124 & 0.566929 & 0.598425 \\
\hline $5 \%$ & 325.976416 & 35.225583 & 333.726471 & 35.225583 & 0.581395 & 0.581395 \\
\hline $10 \%$ & 333.123579 & 28.078420 & 333.966487 & 28.078420 & 0.578125 & 0.593750 \\
\hline $15 \%$ & 334.761387 & 26.440612 & 335.328621 & 26.440612 & 0.581967 & 0.590164 \\
\hline $20 \%$ & 335.612708 & 25.589291 & 335.719509 & 25.589291 & 0.589744 & 0.623932 \\
\hline $25 \%$ & 336.434131 & 24.767868 & 336.167733 & 24.767868 & 0.578947 & 0.578947 \\
\hline $30 \%$ & 337.559031 & 23.642969 & 337.319073 & 23.642969 & 0.601770 & 0.610619 \\
\hline $35 \%$ & 337.982363 & 23.219636 & 337.273095 & 23.219636 & 0.594595 & 0.639640 \\
\hline $40 \%$ & 337.880663 & 23.321336 & 337.651415 & 23.321336 & 0.603774 & 0.650943 \\
\hline $45 \%$ & 338.764696 & 22.437303 & 337.913964 & 22.437303 & 0.617647 & 0.627451 \\
\hline $50 \%$ & 339.391511 & 21.810488 & 339.200682 & 21.810488 & 0.617647 & 0.666667 \\
\hline $55 \%$ & 339.245168 & 21.956831 & 338.463058 & 21.956831 & 0.622449 & 0.591837 \\
\hline $60 \%$ & 339.831044 & 21.370955 & 339.927314 & 21.370955 & 0.642105 & 0.673684 \\
\hline $65 \%$ & 340.019656 & 21.182343 & 339.197860 & 21.182343 & 0.630435 & 0.586957 \\
\hline $70 \%$ & 340.513543 & 20.688456 & 340.285381 & 20.688456 & 0.644444 & 0.600000 \\
\hline $75 \%$ & 340.999074 & 20.202925 & 340.838011 & 20.202925 & 0.602410 & 0.614458 \\
\hline $80 \%$ & 341.208700 & 19.993299 & 340.926612 & 19.993299 & 0.640000 & 0.560000 \\
\hline $85 \%$ & 341.410179 & 19.791820 & 341.063875 & 19.791820 & 0.605634 & 0.591549 \\
\hline $90 \%$ & 342.019619 & 19.182380 & 341.798121 & 19.182380 & 0.550000 & 0.550000 \\
\hline $95 \%$ & 342.153272 & 19.048727 & 341.799376 & 19.048727 & 0.640000 & 0.540000 \\
\hline $100 \%$ & 342.077372 & 19.124627 & 341.841587 & 19.124627 & 0.638298 & 0.617021 \\
\hline
\end{tabular}

Table 30: PCA Image Similarity (PSNR) vs. Rank-1 Recognition Rates for Inter-Trial Randomization on Nose Width Modifications

\begin{tabular}{|l|l|l|r|r|r|r|}
\hline Modification & $\begin{array}{c}\text { PSNR(Avg- } \\
\text { CaseA) }\end{array}$ & $\begin{array}{c}\text { PSNR(Avg- } \\
\text { CaseB) }\end{array}$ & $\begin{array}{c}\text { PSNR(Median- } \\
\text { CaseA) }\end{array}$ & $\begin{array}{c}\text { PSNR(Median- } \\
\text { CaseB) }\end{array}$ & $\begin{array}{c}\text { R1 Rate } \\
\text { (Baseline) }\end{array}$ & $\begin{array}{c}\text { R1 Rate } \\
\text { (Treatment) }\end{array}$ \\
\hline $\mathbf{- 2 0 \%}$ & 334.751678 & 26.450321 & 335.283759 & 26.450321 & 0.580645 & 0.596774 \\
\hline $\mathbf{- 1 5 \%}$ & 327.970224 & 33.231775 & 329.853072 & 33.231775 & 0.571429 & 0.579365 \\
\hline $\mathbf{- 1 0 \%}$ & 315.350594 & 45.851405 & 327.050782 & 45.851405 & 0.564885 & 0.603053 \\
\hline $\mathbf{- 5 \%}$ & 222.524746 & 138.677253 & 307.735052 & 138.677253 & 0.563910 & 0.578947 \\
\hline
\end{tabular}




\begin{tabular}{|l|l|l|l|l|l|l|}
\hline $\mathbf{5 \%}$ & 335.254793 & 25.947206 & 335.336610 & 25.947206 & 0.566929 & 0.566929 \\
\hline $\mathbf{1 0 \%}$ & 335.564236 & 25.637763 & 335.740212 & 25.637763 & 0.571429 & 0.587302 \\
\hline $\mathbf{1 5 \%}$ & 336.498579 & 24.703421 & 336.407225 & 24.703421 & 0.564516 & 0.564516 \\
\hline $\mathbf{2 0 \%}$ & 338.222496 & 22.979503 & 338.345672 & 22.979503 & 0.590164 & 0.614754 \\
\hline
\end{tabular}

Table 31: PCA Image Similarity (PSNR) vs. Rank-1 Recognition Rates for Inter-Trial Randomization on Nose Length Modifications

\begin{tabular}{|c|c|c|c|c|c|c|}
\hline Modification & $\begin{array}{l}\text { PSNR(Avg- } \\
\text { CaseA) }\end{array}$ & $\begin{array}{l}\text { PSNR(Avg- } \\
\text { CaseB) }\end{array}$ & $\begin{array}{l}\text { PSNR(Median- } \\
\text { CaseA) }\end{array}$ & $\begin{array}{l}\text { PSNR(Median- } \\
\text { CaseB) }\end{array}$ & $\begin{array}{l}\text { R1 Rate } \\
\text { (Baseline) }\end{array}$ & $\begin{array}{l}\text { R1 Rate } \\
\text { (Treatment) }\end{array}$ \\
\hline$-100 \%$ & 335.973784 & 25.228215 & 335.677751 & 25.228215 & 0.731707 & 0.772358 \\
\hline$-95 \%$ & 336.346249 & 24.855750 & 336.725338 & 24.855750 & 0.640625 & 0.632813 \\
\hline$-90 \%$ & 335.761879 & 25.440120 & 335.447471 & 25.440120 & 0.672000 & 0.688000 \\
\hline$-85 \%$ & 336.657016 & 24.544983 & 336.764437 & 24.544983 & 0.634921 & 0.619048 \\
\hline$-80 \%$ & 336.430719 & 24.771280 & 336.721314 & 24.771280 & 0.750000 & 0.750000 \\
\hline$-75 \%$ & 336.328224 & 24.873775 & 336.656558 & 24.873775 & 0.733871 & 0.741935 \\
\hline$-70 \%$ & 336.027899 & 25.174100 & 336.606534 & 25.174100 & 0.672131 & 0.729508 \\
\hline$-65 \%$ & 335.888132 & 25.313867 & 335.921267 & 25.313867 & 0.716535 & 0.716535 \\
\hline$-60 \%$ & 336.514409 & 24.687590 & 336.139816 & 24.687590 & 0.669355 & 0.653226 \\
\hline$-55 \%$ & 336.503123 & 24.698876 & 336.908068 & 24.698876 & 0.692913 & 0.732283 \\
\hline$-50 \%$ & 336.522069 & 24.679930 & 336.283730 & 24.679930 & 0.778689 & 0.778689 \\
\hline$-45 \%$ & 335.940268 & 25.261731 & 335.910140 & 25.261731 & 0.546875 & 0.562500 \\
\hline$-40 \%$ & 335.759702 & 25.442298 & 336.229003 & 25.442298 & 0.740157 & 0.732283 \\
\hline$-35 \%$ & 335.324316 & 25.877683 & 335.855507 & 25.877683 & 0.587786 & 0.587786 \\
\hline$-30 \%$ & 335.028942 & 26.173057 & 335.790100 & 26.173057 & 0.656489 & 0.648855 \\
\hline$-25 \%$ & 334.782559 & 26.419440 & 335.550314 & 26.419440 & 0.648000 & 0.624000 \\
\hline$-20 \%$ & 333.782352 & 27.419647 & 334.904847 & 27.419647 & 0.617188 & 0.632813 \\
\hline$-15 \%$ & 332.176206 & 29.025793 & 333.804425 & 29.025793 & 0.720930 & 0.728682 \\
\hline$-10 \%$ & 331.003179 & 30.198820 & 333.672680 & 30.198820 & 0.646154 & 0.676923 \\
\hline$-5 \%$ & 326.009008 & 35.192991 & 333.701013 & 35.192991 & 0.641221 & 0.671756 \\
\hline $5 \%$ & 322.916381 & 38.285618 & 333.508029 & 38.285618 & 0.666667 & 0.682171 \\
\hline $10 \%$ & 332.729451 & 28.472549 & 333.837256 & 28.472549 & 0.715385 & 0.692308 \\
\hline $15 \%$ & 334.350469 & 26.851530 & 335.350233 & 26.851530 & 0.705882 & 0.705882 \\
\hline
\end{tabular}




\begin{tabular}{|c|c|c|c|c|c|c|}
\hline $20 \%$ & 336.225406 & 24.976593 & 336.169169 & 24.976593 & 0.700855 & 0.683761 \\
\hline $25 \%$ & 336.514557 & 24.687443 & 336.527253 & 24.687443 & 0.685185 & 0.675926 \\
\hline $30 \%$ & 337.255317 & 23.946682 & 336.912290 & 23.946682 & 0.754545 & 0.718182 \\
\hline $35 \%$ & 338.144965 & 23.057034 & 337.664129 & 23.057034 & 0.725664 & 0.707965 \\
\hline $40 \%$ & 337.826199 & 23.375800 & 337.485158 & 23.375800 & 0.654545 & 0.645455 \\
\hline $45 \%$ & 338.655496 & 22.546503 & 337.852128 & 22.546503 & 0.711538 & 0.673077 \\
\hline $50 \%$ & 339.078189 & 22.123810 & 339.264841 & 22.123810 & 0.647059 & 0.588235 \\
\hline $55 \%$ & 338.778766 & 22.423233 & 338.426871 & 22.423233 & 0.673267 & 0.643564 \\
\hline $60 \%$ & 339.683982 & 21.518017 & 339.784468 & 21.518017 & 0.616162 & 0.616162 \\
\hline $65 \%$ & 339.976508 & 21.225491 & 339.515400 & 21.225491 & 0.678571 & 0.583333 \\
\hline $70 \%$ & 340.717306 & 20.484693 & 340.353531 & 20.484693 & 0.620690 & 0.482759 \\
\hline $75 \%$ & 341.176406 & 20.025593 & 340.643883 & 20.025593 & 0.671053 & 0.578947 \\
\hline $80 \%$ & 341.532383 & 19.669616 & 340.750308 & 19.669616 & 0.573333 & 0.426667 \\
\hline $85 \%$ & 341.659506 & 19.542493 & 340.848994 & 19.542493 & 0.619718 & 0.408451 \\
\hline $90 \%$ & 341.909935 & 19.292064 & 341.777256 & 19.292064 & 0.576271 & 0.457627 \\
\hline $95 \%$ & 342.677546 & 18.524453 & 341.763860 & 18.524453 & 0.803279 & 0.409836 \\
\hline $100 \%$ & 342.665981 & 18.536018 & 342.042896 & 18.536018 & 0.770833 & 0.500000 \\
\hline
\end{tabular}

Table 32:LBPH Image Similarity (PSNR) vs. Rank-1 Recognition Rates for Intra-Trial Randomization on Nose Width Modifications

\begin{tabular}{|l|c|r|r|r|r|r|}
\hline Modification & $\begin{array}{c}\text { PSNR(Avg- } \\
\text { CaseA) }\end{array}$ & $\begin{array}{c}\text { PSNR(Avg- } \\
\text { CaseB) }\end{array}$ & $\begin{array}{c}\text { PSNR(Median- } \\
\text { CaseA) }\end{array}$ & $\begin{array}{c}\text { PSNR(Median- } \\
\text { CaseB) }\end{array}$ & $\begin{array}{c}\text { R1 Rate } \\
\text { (Baseline) }\end{array}$ & $\begin{array}{c}\text { R1 Rate } \\
\text { (Treatment) }\end{array}$ \\
\hline $\mathbf{- 2 0 \%}$ & 335.104787 & 26.097212 & 335.612245 & 26.097212 & 0.731707 & 0.756098 \\
\hline $\mathbf{- 1 5 \%}$ & 328.200841 & 33.001158 & 330.089530 & 33.001158 & 0.671875 & 0.695313 \\
\hline $\mathbf{- 1 0 \%}$ & 309.010396 & 52.191604 & 328.262904 & 52.191604 & 0.714286 & 0.699248 \\
\hline $\mathbf{- 5 \%}$ & 232.182538 & 129.019461 & 310.048283 & 129.019461 & 0.676692 & 0.676692 \\
\hline $\mathbf{5 \%}$ & 332.101874 & 29.100125 & 335.168749 & 29.100125 & 0.588710 & 0.580645 \\
\hline $\mathbf{1 0} \%$ & 335.666938 & 25.535061 & 336.389255 & 25.535061 & 0.608000 & 0.608000 \\
\hline $\mathbf{1 5 \%}$ & 336.322316 & 24.879683 & 336.027011 & 24.879683 & 0.656250 & 0.625000 \\
\hline $\mathbf{2 0} \%$ & 337.719801 & 23.482198 & 338.271435 & 23.482198 & 0.725806 & 0.701613 \\
\hline
\end{tabular}

Table 33:LBPH Image Similarity (PSNR) vs. Rank-1 Recognition Rates for Intra-Trial Randomization on Nose Length Modifications 


\begin{tabular}{|c|c|c|c|c|c|c|}
\hline Modification & $\begin{array}{l}\text { PSNR(Avg- } \\
\text { CaseA) }\end{array}$ & $\begin{array}{l}\text { PSNR(Avg- } \\
\text { CaseB) }\end{array}$ & $\begin{array}{l}\text { PSNR(Median- } \\
\text { CaseA) }\end{array}$ & $\begin{array}{l}\text { PSNR(Median- } \\
\text { CaseB) }\end{array}$ & $\begin{array}{l}\text { R1 Rate } \\
\text { (Baseline) }\end{array}$ & $\begin{array}{c}\text { R1 Rate } \\
\text { (Treatment) }\end{array}$ \\
\hline$-100 \%$ & 336.437413 & 24.764586 & 336.208631 & 24.764586 & 0.734375 & 0.734375 \\
\hline$-95 \%$ & 336.308018 & 24.893981 & 336.258038 & 24.893981 & 0.726563 & 0.710938 \\
\hline$-90 \%$ & 335.992412 & 25.209587 & 335.584422 & 25.209587 & 0.736000 & 0.712000 \\
\hline$-85 \%$ & 336.649904 & 24.552095 & 336.903301 & 24.552095 & 0.728000 & 0.704000 \\
\hline$-80 \%$ & 336.418780 & 24.783219 & 336.430099 & 24.783219 & 0.725806 & 0.701613 \\
\hline$-75 \%$ & 336.901975 & 24.300024 & 336.828620 & 24.300024 & 0.728000 & 0.664000 \\
\hline$-70 \%$ & 336.171176 & 25.030823 & 335.962090 & 25.030823 & 0.730159 & 0.698413 \\
\hline$-65 \%$ & 335.957536 & 25.244463 & 335.922119 & 25.244463 & 0.728000 & 0.672000 \\
\hline$-60 \%$ & 336.421276 & 24.780723 & 335.819573 & 24.780723 & 0.730159 & 0.706349 \\
\hline$-55 \%$ & 336.445274 & 24.756725 & 336.394887 & 24.756725 & 0.733871 & 0.766129 \\
\hline$-50 \%$ & 336.205694 & 24.996305 & 335.850745 & 24.996305 & 0.728000 & 0.744000 \\
\hline$-45 \%$ & 336.009181 & 25.192818 & 335.809539 & 25.192818 & 0.728000 & 0.704000 \\
\hline$-40 \%$ & 336.207064 & 24.994935 & 336.353902 & 24.994935 & 0.722222 & 0.690476 \\
\hline$-35 \%$ & 335.432531 & 25.769469 & 335.855507 & 25.769469 & 0.724409 & 0.692913 \\
\hline$-30 \%$ & 335.606619 & 25.595380 & 335.927912 & 25.595380 & 0.724409 & 0.708661 \\
\hline$-25 \%$ & 334.475011 & 26.726988 & 335.081198 & 26.726988 & 0.710938 & 0.703125 \\
\hline$-20 \%$ & 333.721523 & 27.480476 & 334.883881 & 27.480476 & 0.705426 & 0.697674 \\
\hline$-15 \%$ & 332.827551 & 28.374448 & 334.229201 & 28.374448 & 0.710938 & 0.687500 \\
\hline$-10 \%$ & 331.862326 & 29.339673 & 334.143722 & 29.339673 & 0.705426 & 0.682171 \\
\hline$-5 \%$ & 323.766875 & 37.435124 & 333.895909 & 37.435124 & 0.708661 & 0.708661 \\
\hline $5 \%$ & 325.976416 & 35.225583 & 333.726471 & 35.225583 & 0.736434 & 0.736434 \\
\hline $10 \%$ & 333.123579 & 28.078420 & 333.966487 & 28.078420 & 0.734375 & 0.726563 \\
\hline $15 \%$ & 334.761387 & 26.440612 & 335.328621 & 26.440612 & 0.737705 & 0.737705 \\
\hline $20 \%$ & 335.612708 & 25.589291 & 335.719509 & 25.589291 & 0.735043 & 0.726496 \\
\hline $25 \%$ & 336.434131 & 24.767868 & 336.167733 & 24.767868 & 0.728070 & 0.684211 \\
\hline $30 \%$ & 337.559031 & 23.642969 & 337.319073 & 23.642969 & 0.743363 & 0.681416 \\
\hline $35 \%$ & 337.982363 & 23.219636 & 337.273095 & 23.219636 & 0.738739 & 0.684685 \\
\hline $40 \%$ & 337.880663 & 23.321336 & 337.651415 & 23.321336 & 0.726415 & 0.726415 \\
\hline $45 \%$ & 338.764696 & 22.437303 & 337.913964 & 22.437303 & 0.725490 & 0.696078 \\
\hline
\end{tabular}




\begin{tabular}{|l|l|l|l|l|l|l|}
\hline $\mathbf{5 0} \%$ & 339.391511 & 21.810488 & 339.200682 & 21.810488 & 0.745098 & 0.656863 \\
\hline $\mathbf{5 5 \%}$ & 339.245168 & 21.956831 & 338.463058 & 21.956831 & 0.734694 & 0.653061 \\
\hline $\mathbf{6 0} \%$ & 339.831044 & 21.370955 & 339.927314 & 21.370955 & 0.736842 & 0.631579 \\
\hline $\mathbf{6 5 \%}$ & 340.019656 & 21.182343 & 339.197860 & 21.182343 & 0.717391 & 0.619565 \\
\hline $\mathbf{7 0} \%$ & 340.513543 & 20.688456 & 340.285381 & 20.688456 & 0.733333 & 0.600000 \\
\hline $\mathbf{7 5 \%}$ & 340.999074 & 20.202925 & 340.838011 & 20.202925 & 0.686747 & 0.566265 \\
\hline $\mathbf{8 0} \%$ & 341.208700 & 19.993299 & 340.926612 & 19.993299 & 0.693333 & 0.560000 \\
\hline $\mathbf{8 5} \%$ & 341.410179 & 19.791820 & 341.063875 & 19.791820 & 0.661972 & 0.521127 \\
\hline $\mathbf{9 0} \%$ & 342.019619 & 19.182380 & 341.798121 & 19.182380 & 0.600000 & 0.500000 \\
\hline $\mathbf{9 5} \%$ & 342.153272 & 19.048727 & 341.799376 & 19.048727 & 0.680000 & 0.460000 \\
\hline $\mathbf{1 0 0} \%$ & 342.077372 & 19.124627 & 341.841587 & 19.124627 & 0.659574 & 0.531915 \\
\hline
\end{tabular}

Table 34:LBPH Image Similarity (PSNR) vs. Rank-1 Recognition Rates for Inter-Trial Randomization on Nose Width Modifications

\begin{tabular}{|l|c|c|c|c|c|c|}
\hline Modification & $\begin{array}{c}\text { PSNR(Avg- } \\
\text { CaseA) }\end{array}$ & $\begin{array}{c}\text { PSNR(Avg- } \\
\text { CaseB) }\end{array}$ & $\begin{array}{c}\text { PSNR(Median- } \\
\text { CaseA) }\end{array}$ & $\begin{array}{c}\text { PSNR(Median- } \\
\text { CaseB) }\end{array}$ & $\begin{array}{c}\text { R1 Rate } \\
\text { (Baseline) }\end{array}$ & $\begin{array}{c}\text { R1 Rate } \\
\text { (Treatment) }\end{array}$ \\
\hline $\mathbf{- 2 0 \%}$ & 334.751678 & 26.450321 & 335.283759 & 26.450321 & 0.741935 & 0.725806 \\
\hline $\mathbf{- 1 5 \%}$ & 327.970224 & 33.231775 & 329.853072 & 33.231775 & 0.730159 & 0.730159 \\
\hline $\mathbf{- 1 0 \%}$ & 315.350594 & 45.851405 & 327.050782 & 45.851405 & 0.717557 & 0.717557 \\
\hline $\mathbf{- 5 \%}$ & 222.524746 & 138.677253 & 307.735052 & 138.677253 & 0.714286 & 0.729323 \\
\hline $\mathbf{5 \%}$ & 335.254793 & 25.947206 & 335.336610 & 25.947206 & 0.708661 & 0.700787 \\
\hline $\mathbf{1 0} \%$ & 335.564236 & 25.637763 & 335.740212 & 25.637763 & 0.714286 & 0.706349 \\
\hline $\mathbf{1 5 \%}$ & 336.498579 & 24.703421 & 336.407225 & 24.703421 & 0.709677 & 0.677419 \\
\hline $\mathbf{2 0} \%$ & 338.222496 & 22.979503 & 338.345672 & 22.979503 & 0.721311 & 0.704918 \\
\hline
\end{tabular}

Table 35:LBPH Image Similarity (PSNR) vs. Rank-1 Recognition Rates for Inter-Trial Randomization on Nose Length Modifications 


\section{APPENDIX D : EUCLIDEAN DISTANCE}

Only two of the three facial recognition algorithms will be shown, since Section 4.5 looks at LDA.

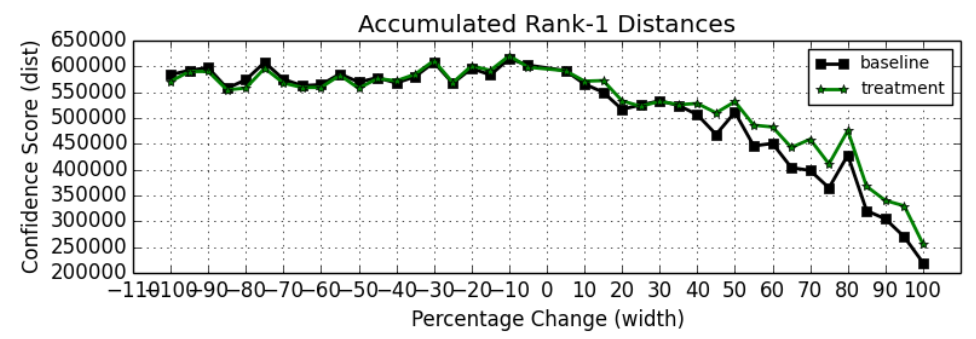

Figure 34: Intra-Trial Randomization Accumulated Distance between Baseline and Treatment Distances for PCA

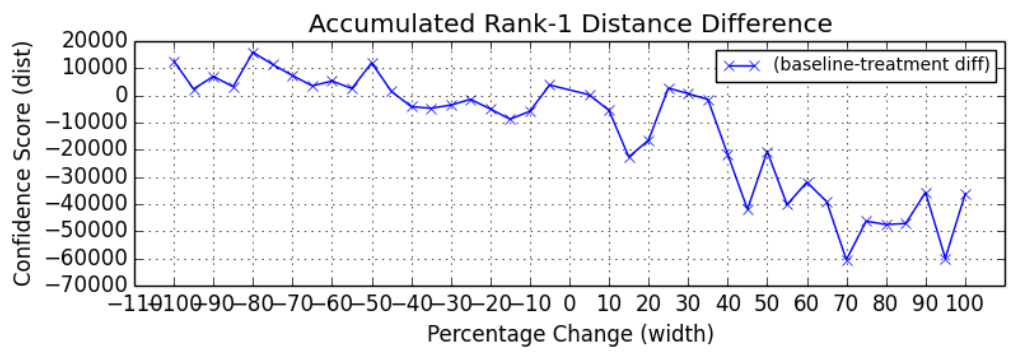

Figure 35: Intra-Trial Randomization Accumulated Difference between Baseline and Treatment Distances for PCA.

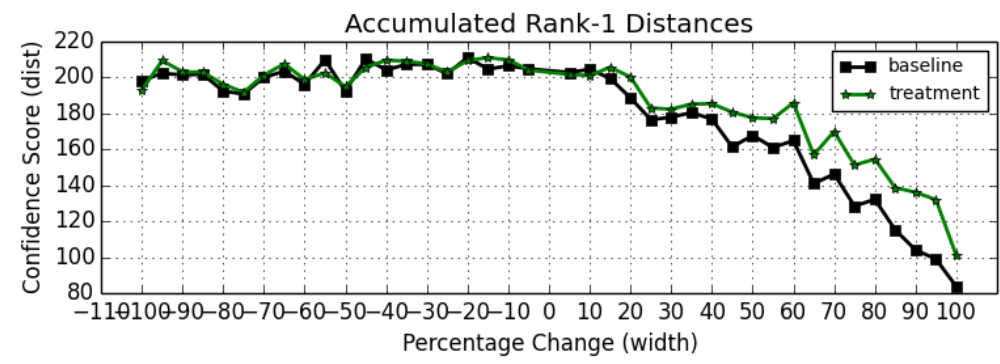

Figure 36: Intra-Trial Randomization Accumulated Baseline and Treatment Distances for LBPH. 


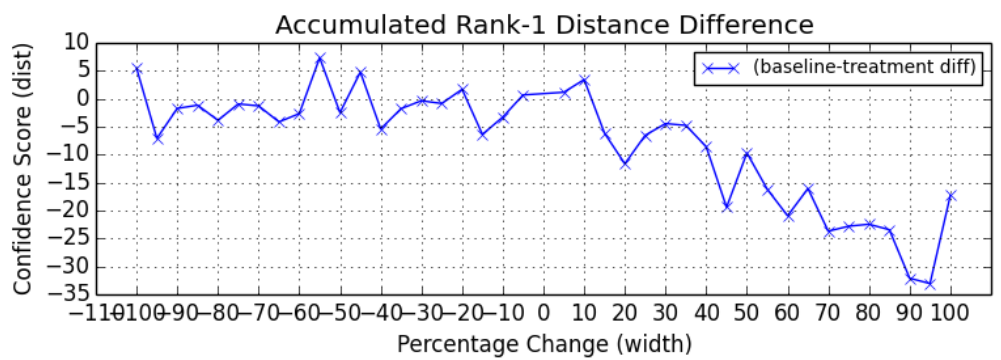

Figure 37: Intra-Trial Accumulated Differences between Baseline and Treatment Distances for LBPH 